\title{
Development of Acetic Acid Removal Technology for the UREX+ Process
}

\author{
Final Report \\ DOE Award Number DE-FC07-06ID14735
}

Chemical and Biomolecular Engineering Department

The University of Tennessee, Knoxville

Principle Investigators

Robert M. Counce

Jack S. Watson

June 30, 2009 


\section{ACKNOWLEDGEMENTS}

This work was supported by the U.S. Department of Energy, Nuclear Energy Research Initiative (NERI) program, under DOE Contract No. DE-PS07-05ID14713. The original period of performance was March 13, 2006 through March 12, 2009; this project was extended at no additional cost through May 31, 2009, Chapters II, III and IV are taken from the Master's Thesis of Jessica Mitchell (2008). Chapters V and VI are from as yet unpublished work by Travis Russell and Jon Garrison. Other student researchers contributing importantly to this project included Jared Johnson and Christina Karni. The principal investigators are especially grateful for the student's innovation and dedication that went into this project.

Jessica A. Mitchel, Removing Acetic Acid from the UREX+ Process, Master's Thesis, University of Tennessee (2008) 


\section{OVERALL ABSTRACT}

It is imperative that acetic acid is removed from a waste stream in the UREX+ process so that nitric acid can be recycled and possible interference with downstream steps can be avoided. Acetic acid arises from acetohydroxamic acid (AHA), and is used to suppress plutonium in the first step of the UREX+ process. Later, it is hydrolyzed into hydroxyl amine nitrate and acetic acid. Many common separation technologies were examined, and solvent extraction was determined to be the best choice under process conditions. Solvents already used in the UREX+ process were then tested to determine if they would be sufficient for the removal of acetic acid. The tributyl phosphate (TBP)-dodecane diluent, used in both UREX and NPEX, was determined to be a solvent system that gave sufficient distribution coefficients for acetic acid in addition to a high separation factor from nitric acid. This solvent system was tested under various TBP concentrations in the dodecane to provide information that can be used for further flow sheet development. The role of water in the acetic acid removal step was quantified in this study and is reported here. The performance of the annual centrifugal contactor for this acetic acid removal step was also quantified and reported here.

Each step in the UREX+ process was examined to determine if there was any acetic acid interference in the performance of any step of the UREX+ flow sheet that would make it necessary to remove the acetic acid prior to that step. It was found that no interference with acetic acid was present. Therefore, the acetic acid removal step can be placed essentially anywhere in the process. For simplicity, it has been proposed to place the removal step at the end of the process after TALSPEAK where all desirable metals have already been extracted and the nitric acid waste stream is prepared to be recycled. 


\section{TABLE OF CONTENTS}

CHAPTER I: INTRODUCTION ............................................................................................. 1

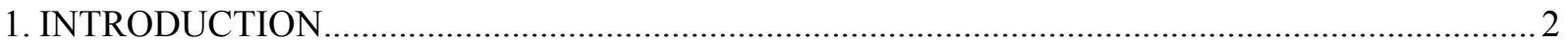

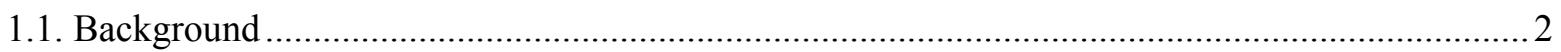

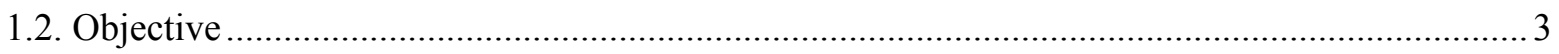

CHAPTER II: REMOVING ACETIC ACID FROM A UREX+ WASTE STREAM: A

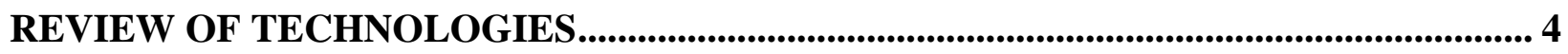

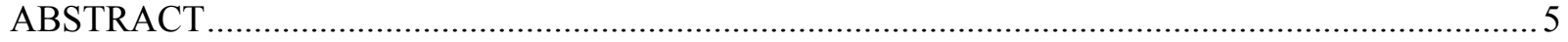

1. INTRODUCTION

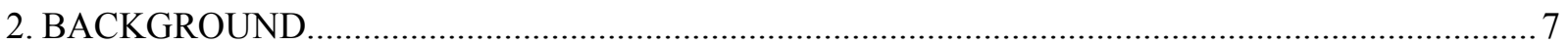

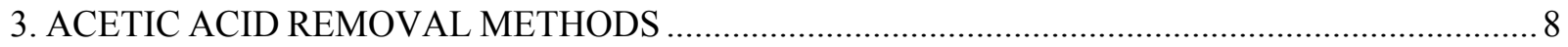

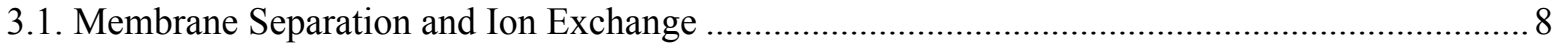

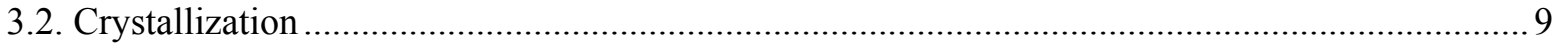

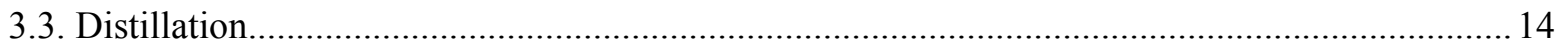

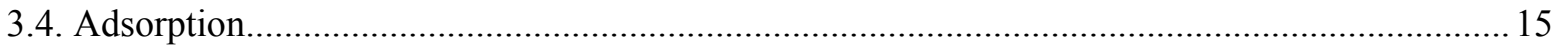

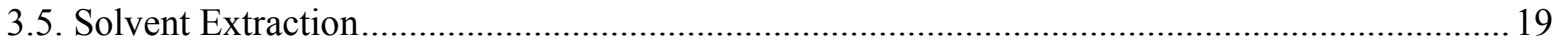

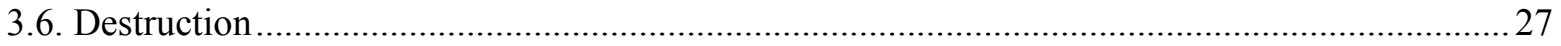

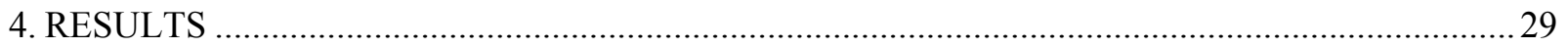

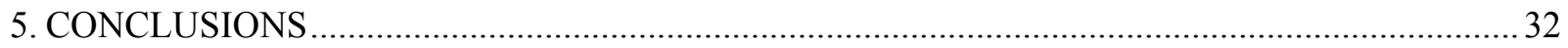

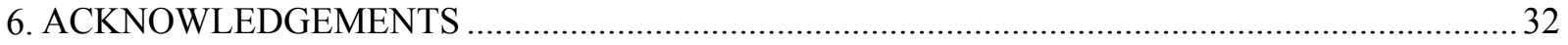

CHAPTER III: EXTRACTING ACETIC ACID FROM ACIDIC SOLUTIONS............... 35

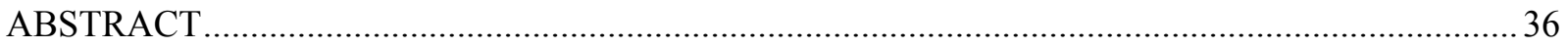

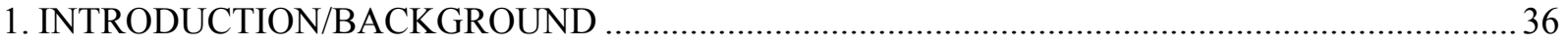

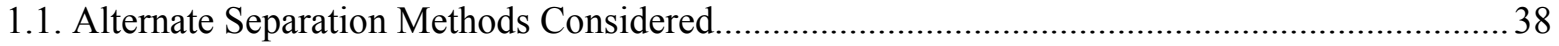




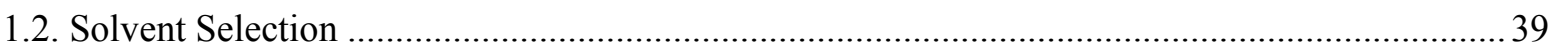

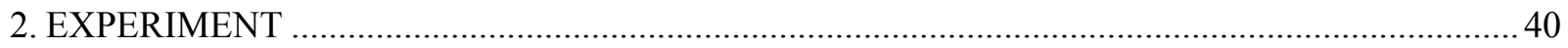

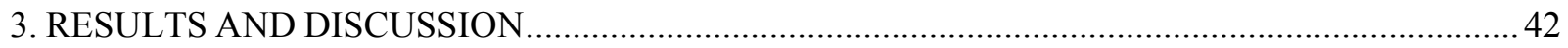

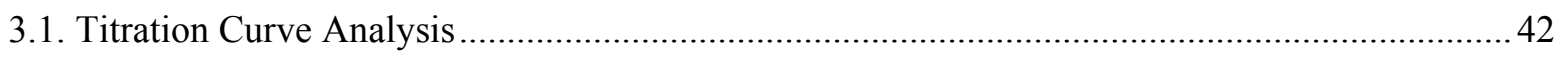

3.2. Acetic Acid Extraction from Water-Acetic Acid Solutions.................................................. 42

3.3. Nitric Acid Extraction from Water-Nitric Acid Solutions ........................................................ 47

3.4. Nitric and Acetic Acid Extraction from Water-Acetic Acid-Nitric Acid Solutions .................... 47

3.5. 2.5M Tributyl Phosphate Mixed With Organic Diluents ......................................................... 48

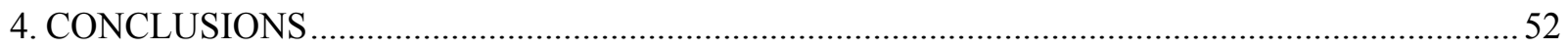

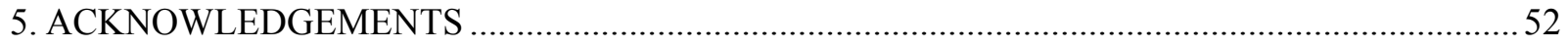

\section{CHAPTER IV: PLACING THE ACETIC ACID STEP INTO THE UREX+ PROCESS.. 55}

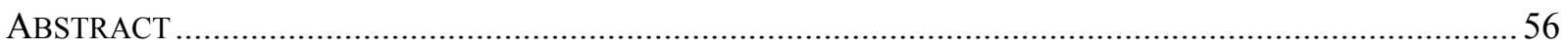

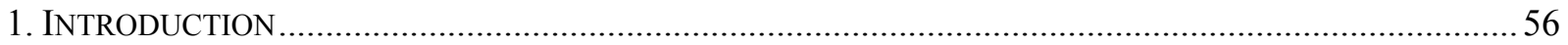

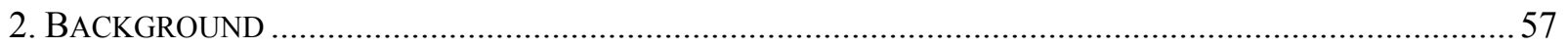

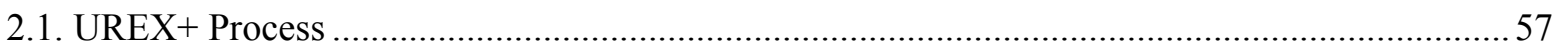

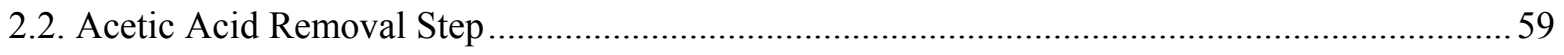

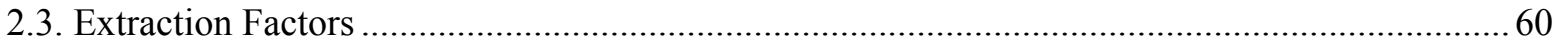

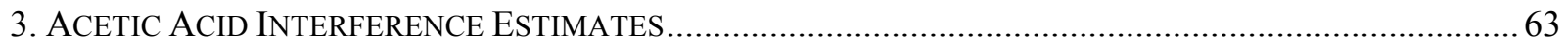

3.1. UREX

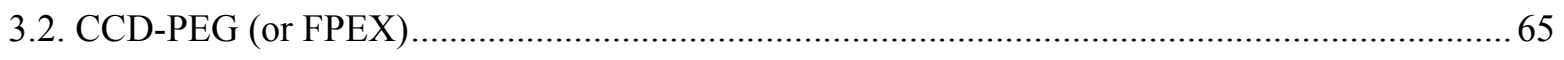

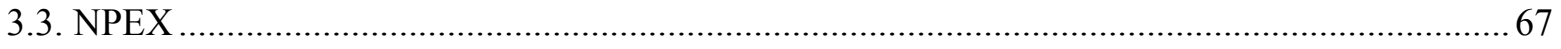

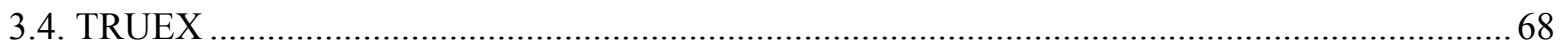

value, the extraction of americium should not be affected by acetic acid to any substantial.............69 


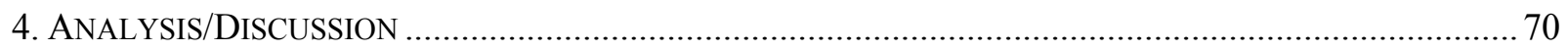

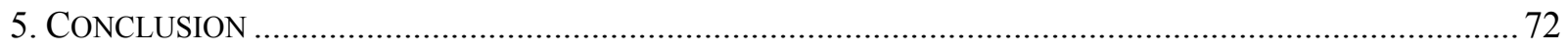

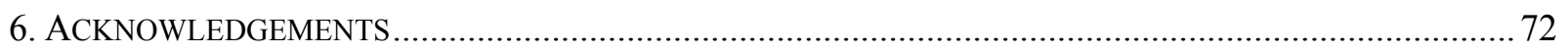

\section{CHAPTER V: WATER SOLUBILITY OF ORGANIC SOLVENTS AND THEIR RELATIONSHIP TO THE EXTRACTION OF NITRIC AND ACETIC ACID FROM}

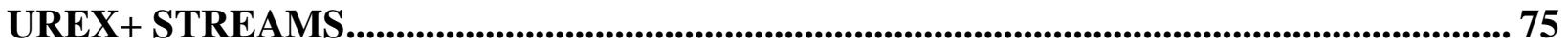

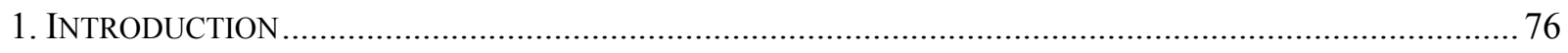

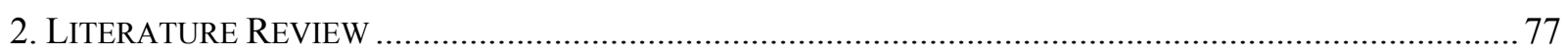

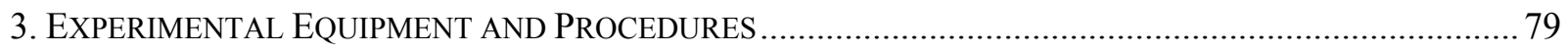

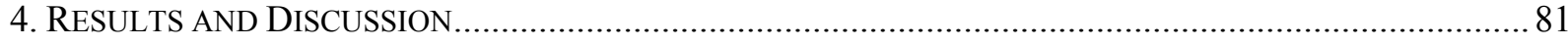

4.1 Water Solubility $\left(\mathrm{C}_{\mathrm{w}, \mathrm{o}}\right)$ Results from Contact Between De-ionized Water and TBP-Organic

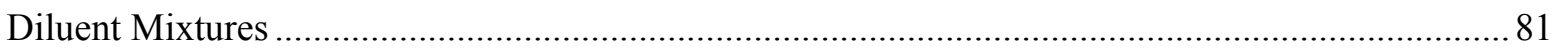

4.2 Water Solubility $\left(\mathrm{C}_{\mathrm{w}, \mathrm{o}}\right)$ Results after Contact with De-ionized Water containing approximately

$0.25 \mathrm{M}$ Nitric Acid and 0.025M Acetic Acid with Various Concentrations of TBP in Organic

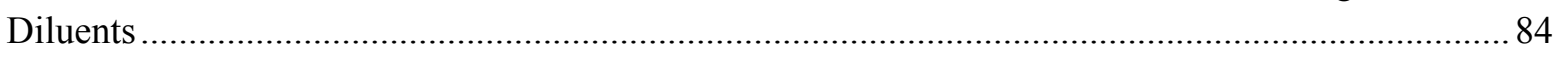

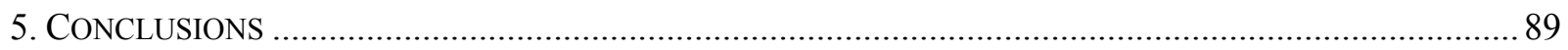

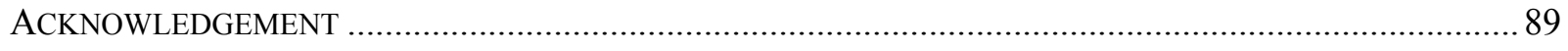

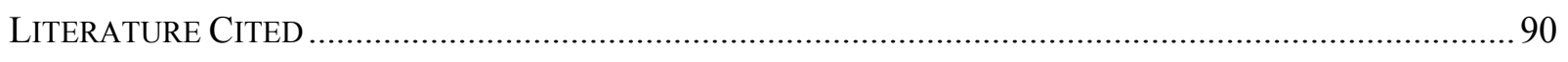

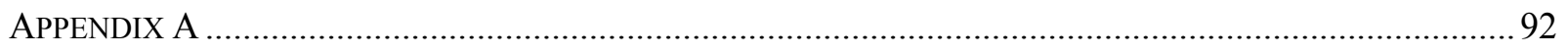

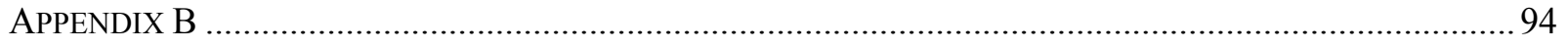

CHAPTER VI: EVALUATION OF CENTRIFUGAL CONTACTORS FOR THE EXTRACTION OF ACETIC ACID FROM UREX+ STREAMS .................................... 96

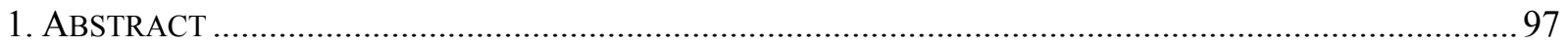

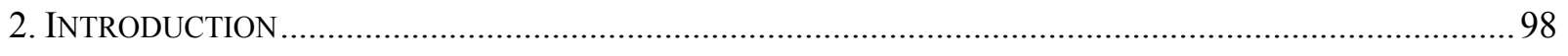

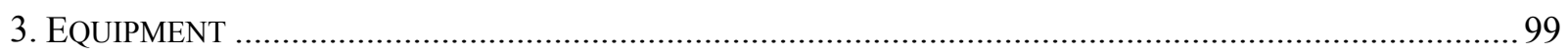




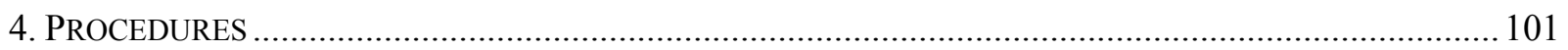

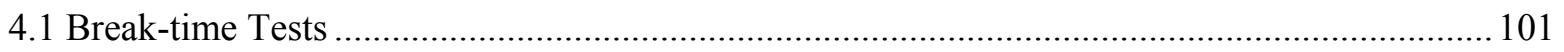

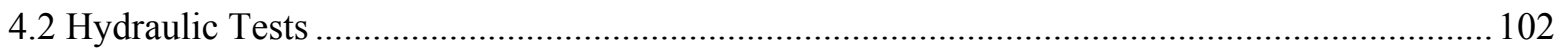

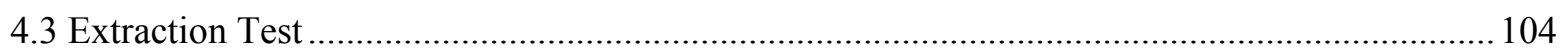

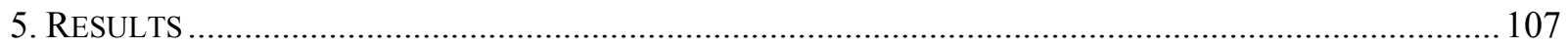

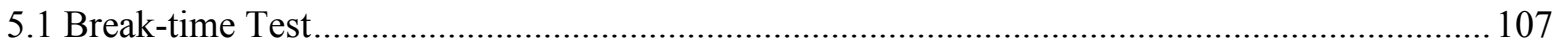

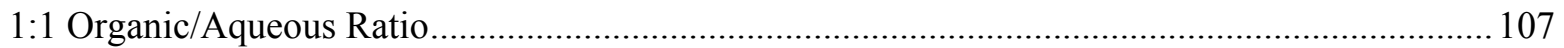

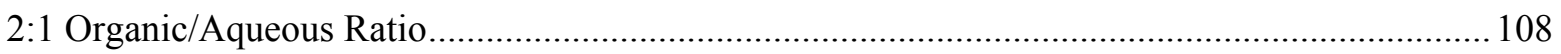

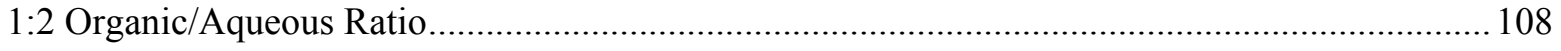

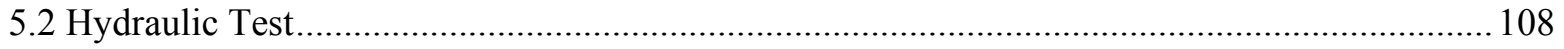

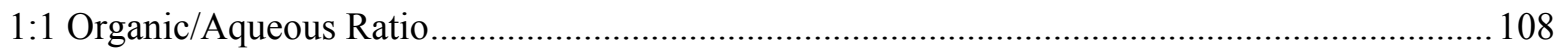

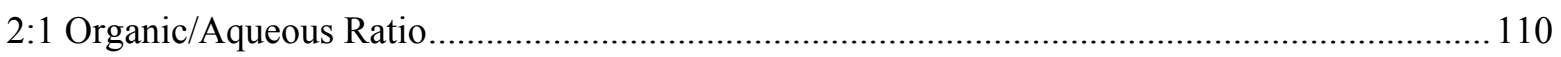

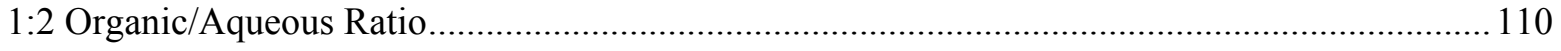

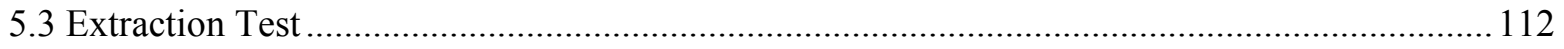

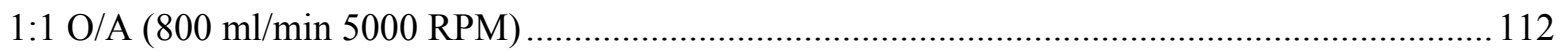

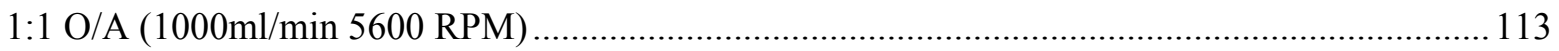

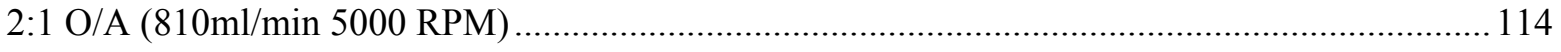

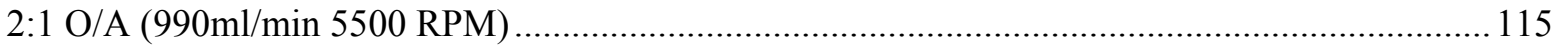

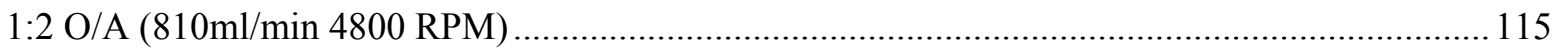

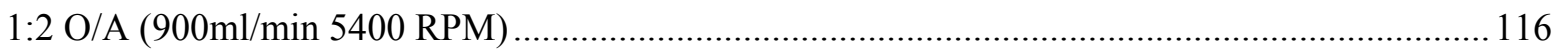

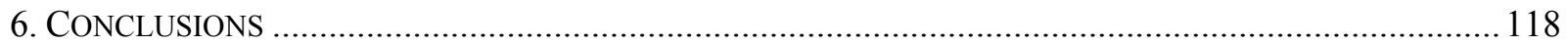

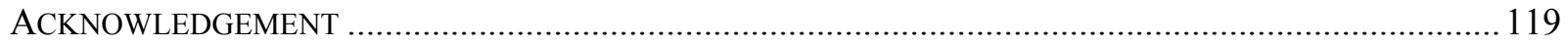

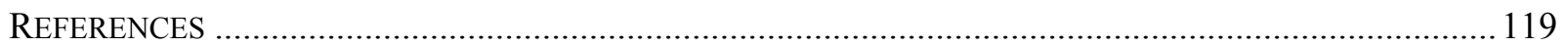




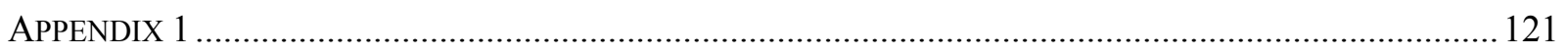

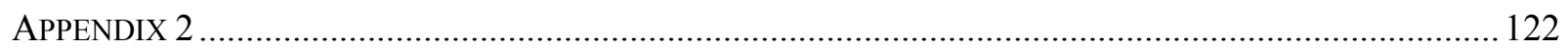

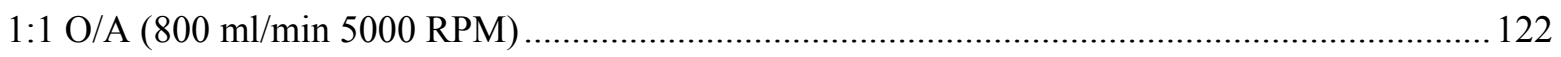

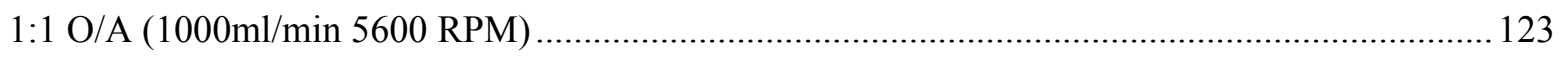

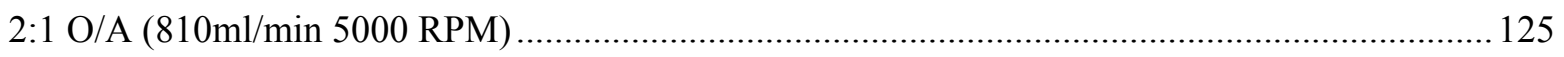

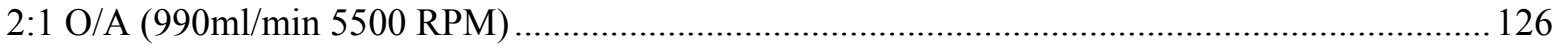

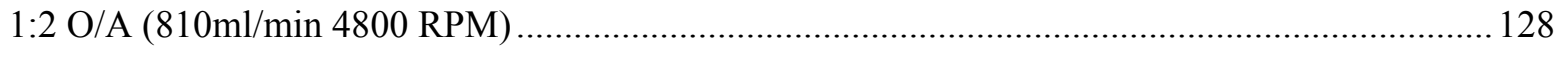

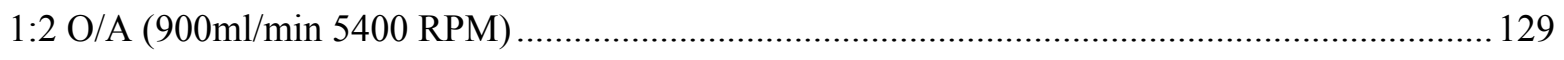

CHAPTER VII: CONCLUSIONS .......................................................................................... 131

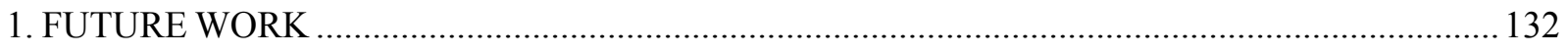

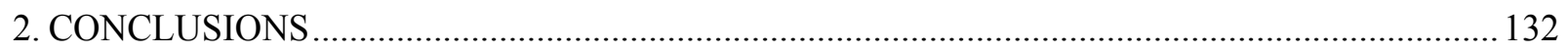

REFERENCES............................................................................................................................ 134 


\section{LIST OF TABLES}

Table 2-1. Physical Properties Error! Bookmark not defined.

Table 2-3: Equilibrium Distribution Coefficients of Acetic Acid between Diluents and Water. [16] ${ }^{\mathrm{a}}$ Chevron Solvent 25 is mostly C-8 and C-9 alkylated aromatics [17] ............................ 21

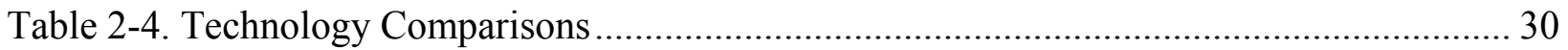

Table 3-1. Extraction of Acetic Acid in Experimental Organic Diluents................................ 46

Table 3-2. Extraction of Acetic Acid in Pure Organic Diluents............................................ 47

Table 3-3. Extraction of Nitric Acid in Pure Organic Diluents. ............................................ 49

Table 3-4. Extraction of Acetic and Nitric Acids in Organic Diluents................................... 50

Table 3-5. Extraction of Acids in 2.5M TBP Mixtures ....................................................... 51

Table 4-1. Distribution Coefficients for the TBP-dodecane Solvent System[1] ...................... 61

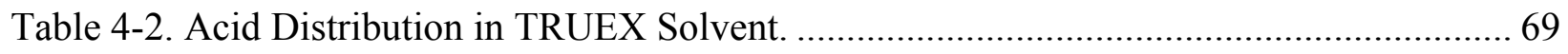

Table 5-1. Equilibrium distribution data for acetic and nitric acid in varying concentrations of

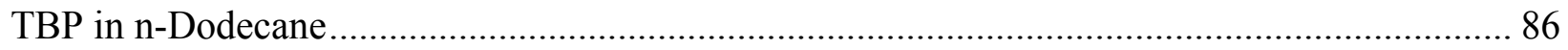

Table 5-2. Water solubility of TBP/n-Dodecane mixture................................................ 92

Table 5-3. Water solubility of TBP/1,2 Dichloroethane mixture ......................................... 92

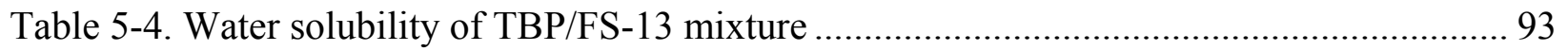

Table 5-5. Water solubility of TBP/n-Dodecane mixture in the presence of the duel acid system

Table 5-6. Water solubility of TBP/1,2 Dichloroethane mixture in the presence of the duel acid

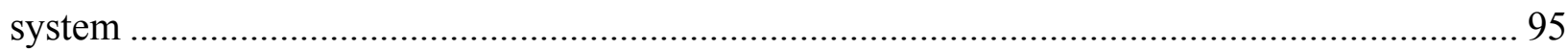

Table 6-1. Batch-test data for 1:1, 1:2, and 2:1 O/A ratios............................................... 121

Table 6-2. Equilibrium test results for a 1:1 organic to aqueous ratio used as a basis for the $800 \mathrm{ml} / \mathrm{min}$ total throughput test on the contactor 
Table 6-3. Sample averages results with standard deviations for $800 \mathrm{ml} / \mathrm{min}$ total throughput test

Table 6-4. Contactor Efficiency for the removal of acetic acid and nitric acid at $800 \mathrm{ml} / \mathrm{min}$ total throughput 123

Table 6-5. Equilibrium test results for a 1:1 organic to aqueous ratio used as a basis for the $1000 \mathrm{ml} / \mathrm{min}$ total throughput test on the contactor

Table 6-6. Sample averages results with standard deviations for $1000 \mathrm{ml} / \mathrm{min}$ total throughput test 124

Table 6-7. Contactor Efficiency for the removal of acetic acid and nitric acid at $1000 \mathrm{ml} / \mathrm{min}$ total throughput 124

Table 6-8. Equilibrium test results for a 2:1 organic to aqueous ratio used as a basis for the $810 \mathrm{ml} / \mathrm{min}$ total throughput test on the contactor

Table 6-9. Sample averages results with standard deviations for $810 \mathrm{ml} / \mathrm{min}$ total throughput test

Table 6-10. Contactor Efficiency for the removal of acetic acid and nitric acid at $810 \mathrm{ml} / \mathrm{min}$ total throughput 126

Table 6-11. Equilibrium test results for a 2:1 organic to aqueous ratio used as a basis for the $990 \mathrm{ml} / \mathrm{min}$ total throughput test on the contactor

Table 6-12. Sample averages results with standard deviations for $990 \mathrm{ml} / \mathrm{min}$ total throughput test

Table 6-13. Contactor Efficiency for the removal of acetic acid and nitric acid at $990 \mathrm{ml} / \mathrm{min}$ total throughput......

Table 6-14. Equilibrium test results for a 1:2 organic to aqueous ratio used as a basis for the $810 \mathrm{ml} / \mathrm{min}$ total throughput test on the contactor.

Table 6-15. Sample averages results with standard deviations for $810 \mathrm{ml} / \mathrm{min}$ total throughput, 1:2 $\mathrm{O} / \mathrm{A}$ test.

Table 6-16. Contactor Efficiency for the removal of acetic acid and nitric acid at $810 \mathrm{ml} / \mathrm{min}$ total throughput, 1:2 $\mathrm{O} / \mathrm{A}$ 
Table 6-17. Equilibrium test results for a 1:2 organic to aqueous ratio used as a basis for the $900 \mathrm{ml} / \mathrm{min}$ total throughput test on the contactor

Table 6-18. Sample averages results with standard deviations for $900 \mathrm{ml} / \mathrm{min}$ total throughput, 1:2 $\mathrm{O} / \mathrm{A}$ test......

Table 6-19. Contactor Efficiency for the removal of acetic acid and nitric acid at $900 \mathrm{ml} / \mathrm{min}$ total throughput, 1:2 O/A 130

\section{LIST OF FIGURES}

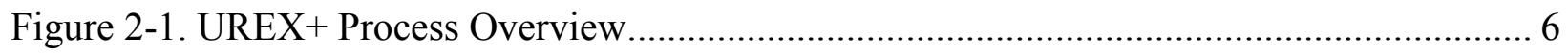

Figure 2-2. Phase Diagram for Acetic Acid and Water. [5] ............................................... 11

Figure 2-3. Phase Diagram for Nitric Acid and Water. [6] ................................................ 12

Figure 2-4. Phase Diagram for Nitric Acid and Acetic Acid. [6] .......................................... 13

Figure 2-5. $\mathrm{K}_{\mathrm{D}}$ as a function of Acetic Acid Concentration using 30\% TBP-Dodecane Solvent with an Organic to Aqueous Ratio of 1. [21] ........................................................................ 24

Figure 2-6. $\mathrm{K}_{\mathrm{D}}$ as a function of Acetic Acid wt\% using TOPO (22 wt\%) in Chevron Solvent 25.

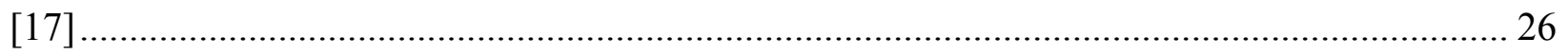

Figure 3-1. UREX+ Flowsheet with Proposed Acetic Acid Removal Step ............................ 37

Figure 3-2. Single Acid Titration Curves (Nitric Acid and Acetic Acid).............................. 44

Figure 3-3. Mixed Acid Titration Curves (Both Nitric and Acetic Acid Together).................. 45

Figure 4-1. The UREX+ Process Used for this Study. [3] ................................................. 58

Figure 4-2. Distribution Coefficients for Acetic Acid in Acetic Acid and Nitric Acid Mixtures vs.

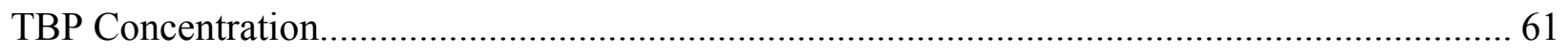

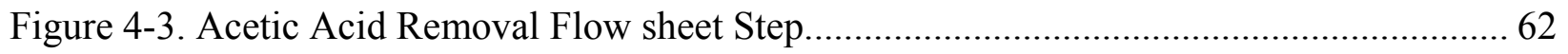


Figure 4-4. Uranium Distribution Coefficients vs. Initial Uranium Concentrations at different

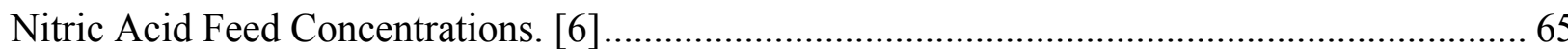

Figure 4-5. Flowsheet Proposal. ..................................................................................... 71

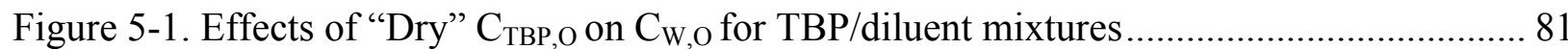

Figure 5-2. Log-log plot of average $\mathrm{C}_{\mathrm{w}, \mathrm{o}}$ of TBP/diluent solutions ..................................... 83

Figure 5-3. Average $\mathrm{C}_{\mathrm{W}, \mathrm{O}}$ of TBP/diluent solutions in the pressence of duel acid system.......... 85

Figure 5-4. Average CW,O of TBP/diluent solutions in the pressence of duel acid system ....... 86

Figure 5-5. Acid extraction data for acetic acid and nitric acid into a TBP/n-Dodecane solution 87

Figure 5-6. Log-log plot of acid extraction data for acetic acid and nitric acid into TBP/n-

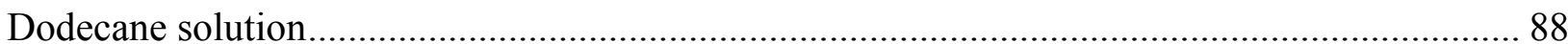

Figure 6-1. Operational Hydraulic Curve for determining points where the contactor fails to

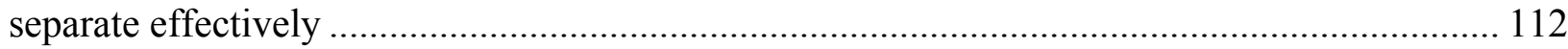

Figure 6-2. Graph showing the results for extraction efficiency tests based on equilibrium

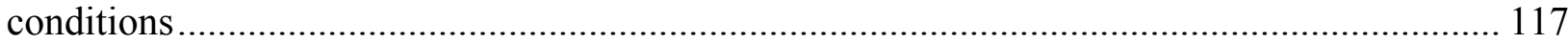

Figure 6-3. Distribution Coefficients taken from contactor extraction test results................... 118 


\section{NOMENCLATURE}

$\mathrm{K}_{\mathrm{D}}=$ Distribution Coefficient

$\mathrm{K}_{\mathrm{C}}=$ Degree of Ionization

$\mathrm{pKa}=$ Acid Dissociation Constant

$[$ Solute $]=$ Concentration of the Solute $(\mathrm{mol} / \mathrm{L})$

$\left[\mathrm{H}^{+}\right]=$Hydronium Concentration $(\mathrm{mol} / \mathrm{L})$

$\left[\mathrm{Ac}^{-}\right]=$Acetate Concentration $(\mathrm{mol} / \mathrm{L})$

$[\mathrm{HAc}]=$ Acetic Acid Concentration $(\mathrm{mol} / \mathrm{L})$

MIBK $=$ Methyl isobutyl ketone

TBP $=$ Tributylphosphate

TOPO $=$ Trioctylphosphine oxide

TnOA $=$ Tri-n-octylamine

$\mathrm{AHA}=$ Acetohydroxamic Aci 


\section{CHAPTER I: Introduction}




\section{INTRODUCTION}

\subsection{Background}

This project is funded by the Advanced Fuel Cycle Initiative (AFCI) as part of the Nuclear Energy Research Initiative (NERI) and the Global Nuclear Energy Partnership (GNEP). The goals of these programs are to establish a fuel reprocessing system capable of recovering fissile materials from spent nuclear fuel for reuse in nuclear power reactors, inhibit the purification of plutonium through co-extraction with actinides, and create an alternative process for recycle of spent nuclear fuel rather than co-extraction in the current ence throughplutonium and uranium extraction (PUREX) method. The production of energy from uranium at an increased industrial level would create a substantial decrease in coal and petroleum dependence for our energy sources. The inhibition of plutonium purification makes it more difficult for plutonium to be used in non-peaceful uses. The recycle of the spent fuel components also creates much less waste for deposition into such repositories as Yucca Mountain.

One process utilizing these objectives is the Uranium Extraction (UREX+) process. The first step in this flow sheet is the Uranium Extraction (UREX) step. This step has an added complexant, acetohydroxamic acid (AHA), which suppresses the extraction of both plutonium and neptunium. The downstream steps of this process vary from flow sheet to flow sheet, but a later step in the process extracts plutonium and neptunium together with minor actinides making plutonium much less attractive for undeclared uses. At the end of this process, the nitric acid waste stream is re-concentrated, most likely through evaporation/distillation, for reuse. This 
recycle of nitric acid requires acetic acid to be removed from the system prior to or during this step to prevent accumulation of acetic acid in the process.

Acetic acid is created from the decomposition of AHA after the UREX step. This hydrolysis is necessary so that plutonium and neptunium can be extracted in a later step, and will probably be accomplished by heating the stream to accelerate the hydrolysis rate. The acetic acid needs to be removed to prevent potential problems accumulation in the recycle of nitric acid and downstream steps.

\subsection{Objective}

The objective of this thesis is to determine a way to remove acetic acid from the raffinate stream of the UREX+ process or downstream in the UREX+ process. This will bewill be explained done in three chapters. The goal of Chapter II is to evaluate the most promising technology through literature review that will remove acetic acid in these process conditions. In Chapter III, the experimental evaluation of the selected technology will be explored. Finally, Chapter IV will determine the placement of this removal step in the UREX+ process. Chapter V provides insight into the behavior of water in aqueous nitric acid-acetic acid systems. Chapter VI provides information on the application of centrifugal contactors for use in removal of acetic acid by solvent extraction. 


\section{CHAPTER II: Removing Acetic Acid from a UREX+ Waste Stream: A Review of Technologies}

Chapter II is a slightly revised version of an article by the same name was published in the journal Nuclear Technology 43 , pp 1- 10 in 2009 by Jessica Mitchell, Robert M. Counce, Jack S. Watson, Barry B. Spencer, and G.D. Del Cul: The main revision is a slight expansion of the discussion of distillation. This expansion was made because one reviewer of a later paper showed that more should have been said about distillation. 


\begin{abstract}
This study explores different technologies for removing acetic acid from a UREX+ waste stream. The waste stream contains both nitric and acetic acids, and the acetic acid must be removed from the waste stream to prevent potential problems in the downstream steps as well as affecting the recycle of nitric acid. The acetic acid is formed after the UREX step of the process as a result of hydrolytic degradation of acetohydroxamic acid used to suppress plutonium extraction. Of the available technologies, the two most attractive approaches are solvent extraction and distillation. In industry, solvent extraction is used for more dilute concentrations of acetic acid while distillation is used for concentrated acetic acid. In this case, it would be necessary to remove most of the water to separate the nitric acid and acetic acid effectively. If a liquid-liquid extraction is viable, this would be the best option with the addition of an extractant, like tributyl phosphate or tri-n-octyl amine, if needed. However, if acetic acid removal can be delayed until the end of the UREX+ process when the nitric acid may be concentrated for recycle, distillation may remain an option, though not necessarily a better option than solvent extraction.
\end{abstract}

\title{
1. INTRODUCTION
}

The objective of this study is to identify and evaluate the most attractive approach to removing acetic acid from the UREX+ process (Figure 2-1). The evaluation includes consideration of acceptability of the approach in nuclear material processing, the effects of the 
approach on the UREX+ process and downstream process steps, as well as factors that affect costs of the approach. Technologies including solvent extraction, destruction, absorption, distillation, and crystallization for the removal/destruction of acetic acid are studied, based on available literature. The objective of this study is to determine the most appropriate method for

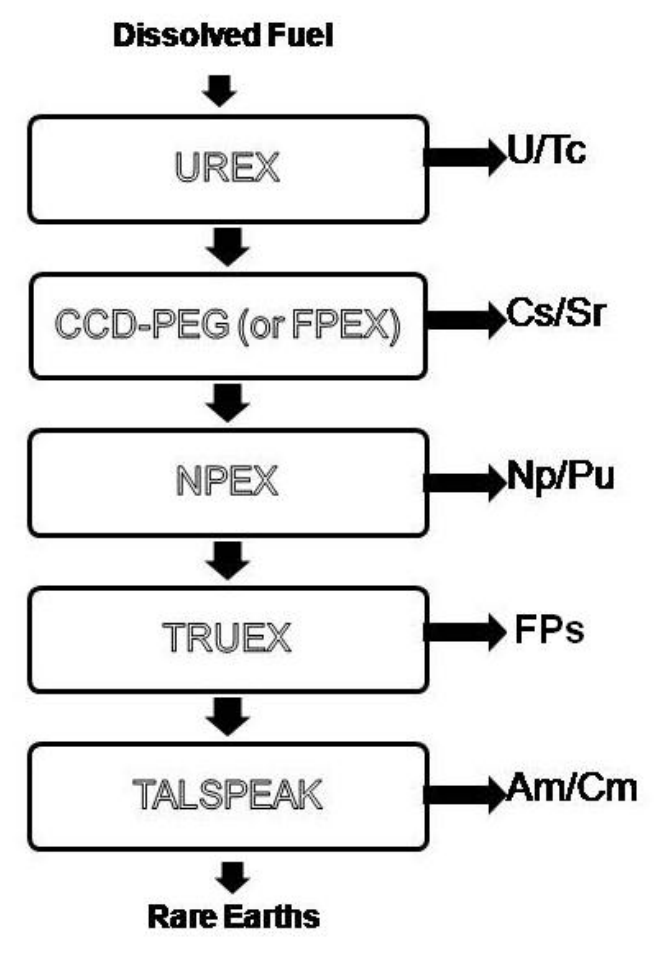

Figure 2-1. UREX+ Process Overview

(1) effective removal of acetic acid without removal of other key components in the process, and

(2) the removal step must not interfere with other downstream steps or the recycling of nitric acid. Once chosen, this method will be experimentally verified at UREX+ process conditions. The degree/percent of removal remains a variable in this study since no specific limit for residual acetic acid was available. 


\section{BACKGROUND}

The UREX+ process, as presented in Figure 2-1, is a series of solvent extraction steps designed to treat spent nuclear fuel by separating its various components for reuse and disposal. Since this process is still under development, there are many different flow sheet scenarios. The flow sheet presented in Figure 2-1 is used in this study.

The first step in Figure 2-1 is the UREX step; nuclear fuel dissolved in aqueous nitric acid is treated with an organic solvent to remove uranium and technetium. The solvent consists of tributyl phosphate dissolved in n-dodecane with acetohydroxamic acid (AHA) added to the aqueous stream to prevent the extraction of plutonium. Both uranium and technetium are extracted into this solvent. ${ }^{1}$ The downstream steps of this process, CCD-PEG (or FPEX), NPEX, TRUEX, and TALSPEAK, are discussed at length in papers on the UREX+ process. [1-4]

The source of the acetic acid, which is the focus of this study, is a degradation product of acetohydroxamic acid. In the acid environment, most of the acetohydroxamic acid hydrolyzes to produce acetic acid and hydroxylamine nitrate. Acetic acid and residual AHA are assumed to leave the UREX segment of the process in the raffinate. The complexant will be completely destroyed prior to the plutonium removal step (NPEX) so that the plutonium will no longer be suppressed. The acetic acid must be separated and/or destroyed because it has potential to interfere with downstream steps and with the recycling of the nitric acid for reuse in the UREX+ process. [3] 
The raffinate stream of interest was modeled as an aqueous mixture of nitric acid and acetic acid in an approximate 10:1 concentration ratio ( $0.5 \mathrm{M}$ nitric acid and $0.05 \mathrm{M}$ acetic acid). This is believed to be close to the acid concentrations in the raffinate streams, but, of course, the concentrations will depend upon where the acetic acid removal step is placed in the UREX+ process. There will also be numerous salts in the raffinate stream, but those are assumed to be at low concentrations and are not expected to affect the acetic acid removal or destruction step. However, the behavior of those salts during the separation and destruction will be an important consideration throughout this analysis of removal options since many of these salts are (1) highly radioactive, (2) desired products to be recovered in downstream steps, or (3) may affect the disposal of any wastes from the acetate removal step.

Relevant physical properties for acetic acid, nitric acid, and water are shown in Table 2-1. Additionally, nitric acid and water form a maximum boiling azeotrope at about 68 weight percent nitric acid The following section summarizes the result of the literature study of potential acetic acid removal methods

Table 2-1. Physical Properties

\begin{tabular}{|c|c|c|c|}
\hline Properties & Acetic Acid $\left(\mathrm{CH}_{3} \mathrm{COOH}\right)$ & Nitric Acid $\left(\mathrm{HNO}_{3}\right)$ & Water $\left(\mathrm{H}_{2} \mathrm{O}\right)$ \\
\hline Boiling Point $\left({ }^{\circ} \mathrm{C}\right)$ & 118 & 83 & 100 \\
\hline Freezing Point $\left({ }^{\circ} \mathrm{C}\right)$ & 16.6 & -42 & 0 \\
\hline Vapor Pressure $\left(\mathrm{mm} \mathrm{Hg} @ 20^{\circ} \mathrm{C}\right)$ & 11 & 48 & 17.5 \\
\hline $\mathrm{pKa}$ & 4.8 & -1.5 & 15.74 \\
\hline
\end{tabular}

3. ACETIC ACID REMOVAL METHODS

\subsection{Membrane Separation and Ion Exchange}


Technologies such as membrane separation and ion exchange were eliminated early in the literature review. No membrane was found to be sufficiently selective to remove acetic acid

in a single pass, and multistage membrane operation was not thought to be an attractive option. Also organic membranes are not normally used in high radiation fields because of potential radiation degradation; so the membrane selection may be limited to inorganic membranes.

Ion exchange was also eliminated as a possible technology because no ion exchange material was identified with sufficient selectivity for acetate ions over nitrate ions at conditions of this study. Ion exchange occurs primarily through adsorption onto a resin with exchangeable ions, anions in this case. For the raffinate stream of interest, nitric acid depresses the dissociation of the acetic acid, i.e. it lowers the acetic acid uptake and reduces the selectivity of anion resins for acetate ions.

\subsection{Crystallization}

Crystallization is the formation of a solid phase from a homogeneous liquid phase. It is of possible interest due to the high freezing point of acetic acid compared to water. Crystallization first requires a saturated solution so that further changes in solution conditions cause solid formation (crystallization). Currently, solubility data are available only for binary systems, water-nitric acid, water-acetic acid, and nitric acid-acetic acid. Information on ternary systems of nitric acid-acetic acid-water are not available, so evaluations were made on three binary systems. 
The acetic acid-water phase diagram (Figure 2-2) shows for dilute acetic acid solutions (to the left of the eutectic) the crystallizing specie is water. Substantial concentration of the aqueous acetic acid-water system must occur before acetic acid becomes the crystallizing specie (to the right of eutectic). The freezing points for nitric acid and water are quite a bit lower than for acetic acid and water (Figure 2-3). Since the solution of interest contains substantial quantities of nitric acid and nitrate salts in addition to acetic acid and water, the presence of this species lead to additional changes in the freezing point. In general, the addition of various salts as well as nitric acid to aqueous acetic acid solutions results in an increase in activity for the acetic acid and a decrease in water activity. [7,8] Applying such information, the freezing point curves will likely shift some for the solution of interest from that shown for the aqueous acetic acid binary system. Other pertinent information comes from Linke and Seidell (1965) who indicate for the acetic acid-nitric acid binary system there is a nitric acid-acetic acid specie with an estimated freezing point of about -23.9 degrees Celsius. While this does not rule out the possibility of a useful acetic acid-nitric acid compound for crystallization, it indicates that considerable concentration of the acetic acid and nitric acid is likely to be necessary, and the eventual solid phase that removes acetic acid is likely to contain some nitric acid. Also, the behavior of dissolved salts during crystallization may be important if crystallization is used for acetic acid removal. Multistage crystallization equipment was noted to usually involve considerable mechanical equipment for washing and redissolving the crystals. The primary conclusion from Figures 2-2, 2-3, and 2-4 is that considerable concentration of acetic acid appears to be necessary before crystallization can be a viable candidate technology for acetic acid removal. Thus crystallization was not considered further in this study. It is noted, however, 


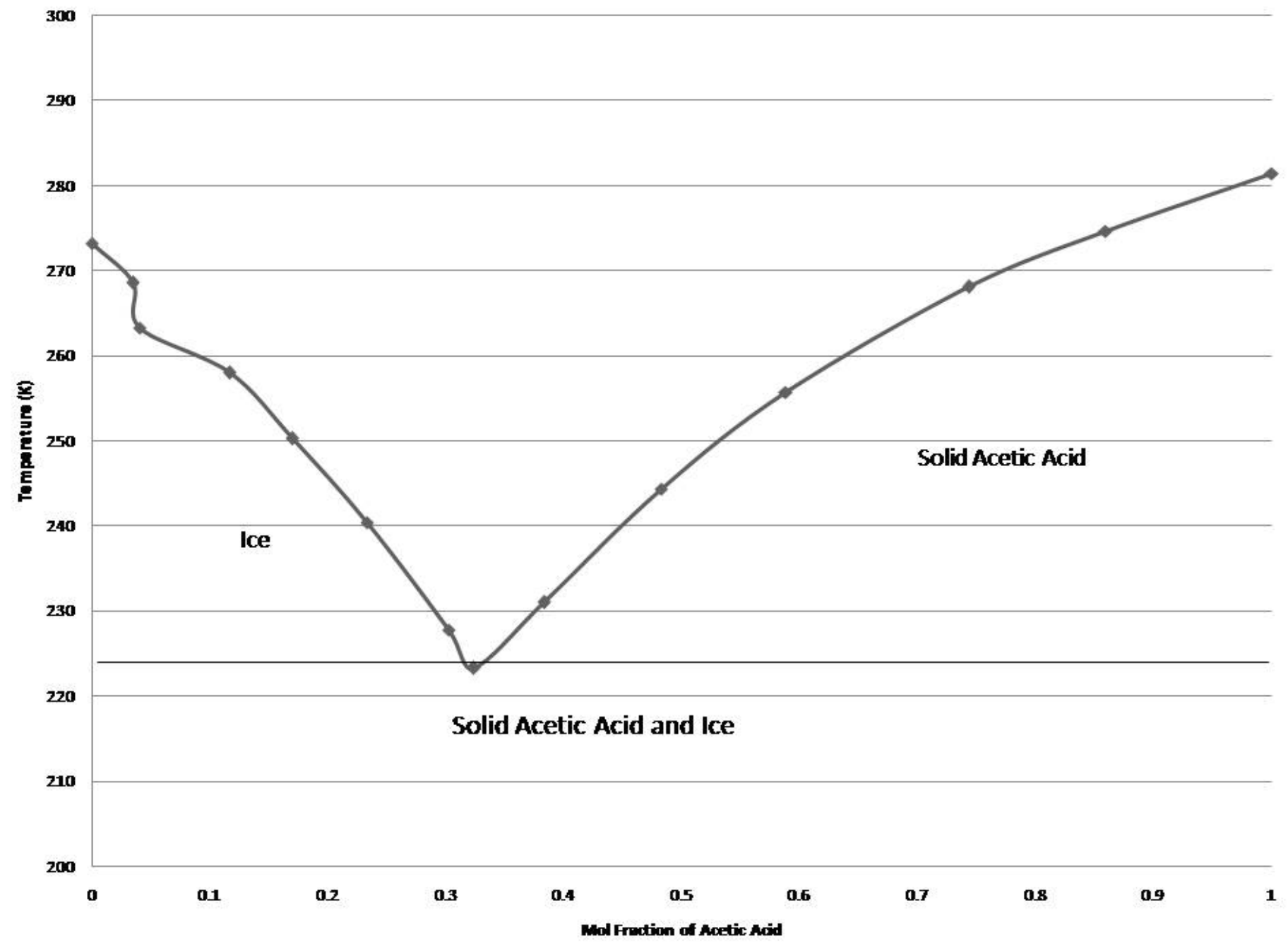

Figure 2-2. Liquid-Solid Phase Diagram for Acetic Acid and Water. [5] 


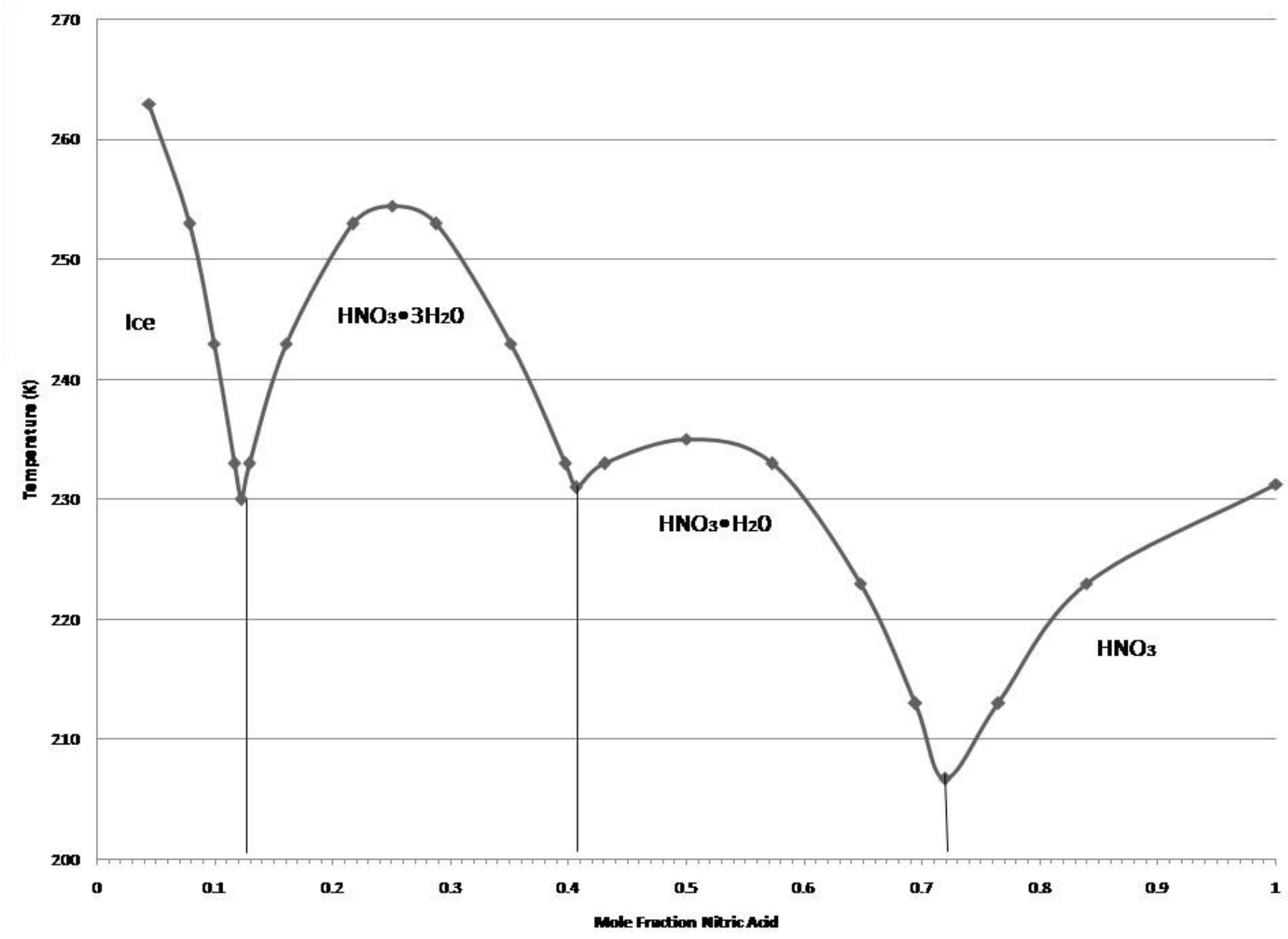

Figure 2-3. Liquid-Solid Phase Diagram for Nitric Acid and Water. [6] 


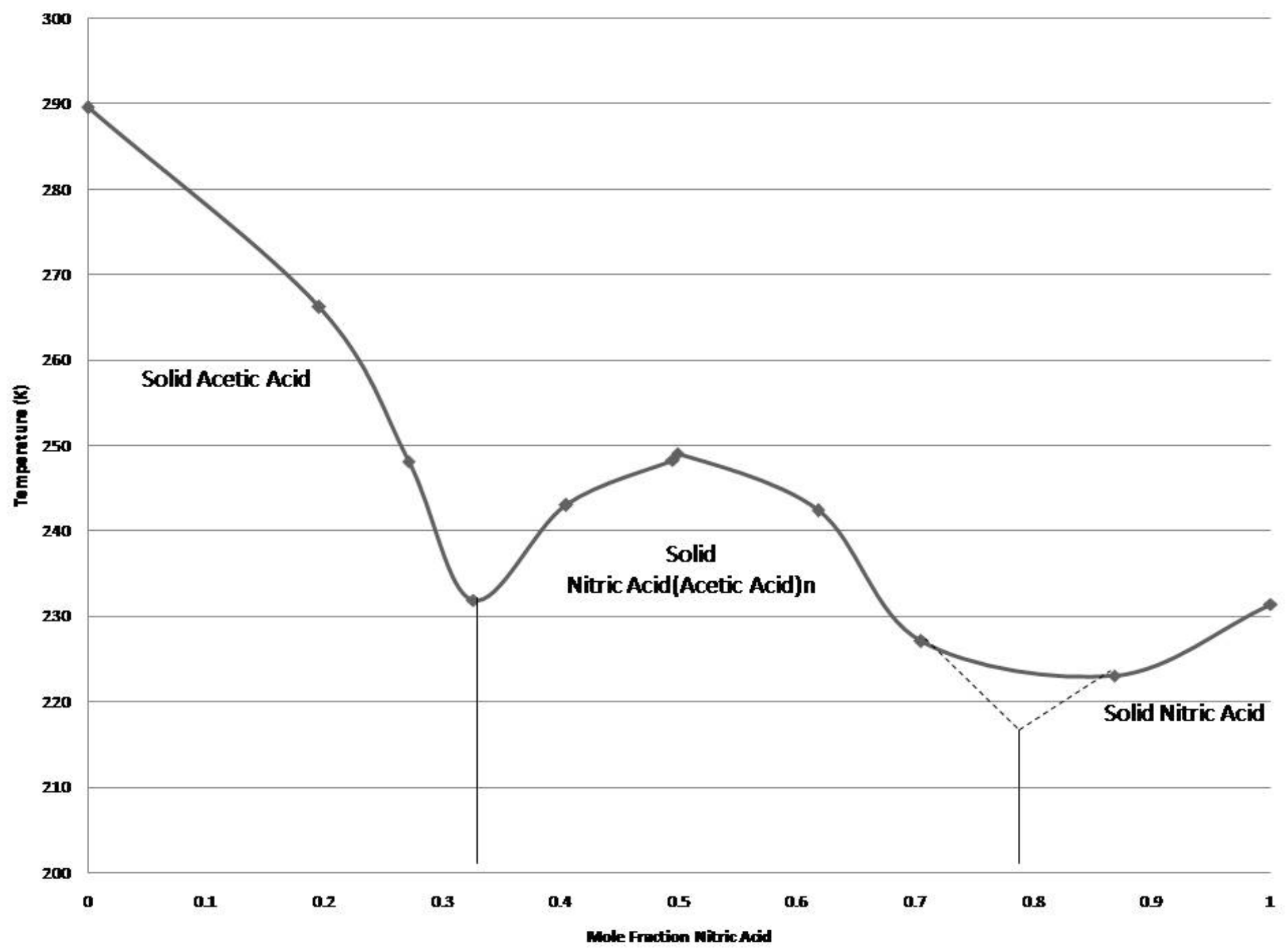

Figure 2-4. Liquid-Solid Phase Diagram for Nitric Acid and Acetic Acid. [6] 
that in activities where nitric acid is concentrated for recycle crystallization of acetic acid may again be of interest.

\subsection{Distillation}

Acetic acid and water have been separated industrially by simple, azeotropic, and extractive distillation. $[9,10]$ The purpose of these industrial uses is to concentrate the acetic acid starting with concentrations higher than $5 \mathrm{wt} \%$ of acetic acid not present in the UREX + process. At these concentrations, the most volatile component is water; so distillation would initially remove water and concentrate the nitric and acetic acids. If distillation is employed for another purpose like nitric acid concentration in the recycle stream, then it may be useful to consider this option more seriously for acetic acid removal. For this reason, further discussion of distillation is needed.

Acetic acid gas-liquid distribution coefficients at 13.3 kBar for the three-component system of nitric acid, water, and acetic acid from Nagahama and Jiang (1989) vary from relative volatilities $\left(\mathrm{K}_{\text {Acetic }} / \mathrm{K}_{\text {water }}\right)$ of 0.594 at a temperature of $329.6 \mathrm{~K}$ to 0.975 at a temperature of 343.8K. Using the UREX model stream of $0.5 \mathrm{M}$ nitric acid with $0.05 \mathrm{M}$ acetic acid, there is very little change in the vapor-liquid equilibrium data from the binary acetic acid-water system to the ternary acetic acid-nitric acid-water system. [7] Thus, distillation would continue to be attractive for higher concentrations of acetic acid, but not so attractive for low concentrations. Distillation (or evaporation) will be used to concentrate nitric acid for reuse. It would also be possible to use distillation to remove acetic acid (separate nitric acid from acetic acid), but that could prove to be 
more difficult than simply concentration of the nitric acid. Without complete vapor-liquid equilibria data for the ternary system (water, nitric acid, and acetic acid), the exact behavior of a distillation system is not known. However, it is evident that most of the water will have to be removed before large portions of the acetic acid can be removed. Trace metal salts in the nitric acid may precipitate in the still. The nitric acid would have to be concentrated almost to the azeotrope composition ( $68 \% \mathrm{wt}$ nitric acid). Even then several distillation states are likely to be needed to separate the acetic acid from the nitric acid azeotrope because the boiling points of the two components are so close. Furthermore, such concentrated nitric is believed to be very corrosive and highly oxidative. This could become an operational hazard if significant quantities of organic compounds should get into this stream, even by accident.

Thus, distillation is likely to be used to concentrate nitric acid sufficiently for recycle, the potential problems with using distillation to remove acetic acid look sufficiently serious to make distillation less attractive than other acetic acid removal methods.

\subsection{Adsorption}

Adsorption involves the transfer of a solute from a fluid phase to a solid surface where it is bound by intermolecular forces. The solute being concentrated on a surface is defined as the adsorbate, and the material on which the adsorbate accumulates is defined as the adsorbent. The amount of material that can be accumulated on a unit area of a surface is small; so useful adsorbents are typically porous material such as activated carbon or other materials with large internal surface areas. The term adsorption as used in this review includes any form of bulk 
uptake by solids, but only one term is used for the sake of simplicity. The design of adsorption equipment requires selection of an adsorbent and information on the equilibrium loading of the adsorbate on the adsorbent (the isotherm), the rate of transport of the adsorbate to the surface during adsorption and away from the surface during regeneration, and equilibrium loading of the adsorbate under regeneration conditions. As part of the process for selection of the adsorbent, the following information is necessary on the characteristics of the adsorbent: (1) the equilibrium capacity of the adsorbent, (2) the selectivity of the adsorbent, (3) physical and chemical characteristics of the adsorbent and (4) the regeneration characteristic of the adsorbent.

Generally the adsorption of weak electrolytes from aqueous solutions occurs through the association of the undissociated molecule with the hydrophobic surface. At conditions where the $\mathrm{pH}$ is lower than the $\mathrm{pKa}$ of the ionizable solute, the equilibrium loading will be greater than that expected at conditions where the $\mathrm{pH}$ is higher than the $\mathrm{pKa}$. Acetic acid in the stream of interest will be largely associated, not ionized, at the expected $\mathrm{pH}$, due to the presence of nitric acid, and the pKa of the acetic acid (see Table 2-1) thereby favors the adsorption of acetic acid in the conditions of this study.

Equilibrium adsorption capacity for acetic acid on various carbons and polymeric adsorbents is widely reported in the literature. In a study utilizing activated carbon with a surface area of $1080 \mathrm{~m}^{2} / \mathrm{g}$, it was determined that the equilibrium capacity for a 0.0333 molar aqueous acetic acid to be 0.081 grams acetic acid per gram of carbon. [11] In a similar study of commercially-available activated carbons with specific surface areas ranging from 390 to 2350 
$\mathrm{m}^{2} / \mathrm{g}$ found capacities for 1 weight percent aqueous solutions of acetic acid to be 0.05 to $0.18 \mathrm{~g}$ acetic acid per g carbon. [12] Several different mathematical forms for expressing the equilibrium data have been used such as the Langmuir isotherm, [13] Frueundlich-type isotherms, [14] and the Radke/Praunsnitz type isotherm. [15]

The term "polymeric adsorbents" is used in this report for synthetic organic adsorbents without functional groups. Adsorption onto polymeric adsorbents without functional groups is a surface-based phenomena similar to that of activated carbon; surface areas of 400 to $1000 \mathrm{~m}^{2} / \mathrm{g}$ are common. The uptake mechanism shifts from surface based phenomena to that of bulk uptake at surface areas of about $500 \mathrm{~m}^{2} / \mathrm{g}$. Kuo et al. (1987) found equilibrium capacities for $1 \mathrm{wt} \%$ acetic acid of up to $0.12 \mathrm{~g}$ acetic acid per $\mathrm{g}$ of adsorbent for several commercial nonfunctionalized adsorbents; this is somewhat lower than similar equilibrium capacities of activated carbon but are comparable when expressed on the basis of specific surface area.

Nitric acid is not likely to be adsorbed to a significant extent on activated carbon or other non-functionalized adsorbents. The possibility of nitration reactions of nitric acid with polymeric adsorbents deserves careful investigation. For activated carbon adsorbents, nitration of carbon could occur after many cycles and possibly lead to the formation of compounds which not only would be unfavorable but could also be a safety concern. The low nitric acid concentration is a favorable factor in reducing the likelihood of significant nitration. Nevertheless, it is likely to be desirable to restrict the useful life of any carbon or polymer based adsorbent to reduce the potential for accumulation of excessive nitration products. 
The usual methods of adsorbent regeneration include stripping at a higher temperature (usually with steam), desorption with a reactive solution (such as an aqueous base), or leaching with an appropriate solvent (such as acetone, various acetates, and methanol). The commercial non-functionalized adsorbents are generally more easily regenerated by solvents than activate carbon. [16] Since acetic acid will be adsorbed as the un-ionized molecule, it may be possible in this case to strip with either a dilute basic (caustic) solution that would ionize the acetic acid or an organic solvent that has favorable acetic acid solubility. 


\subsection{Solvent Extraction}

The removal of a solute from a liquid solution using another immiscible liquid is referred to as liquid-liquid or solvent extraction. Most investigations of solvent extraction express that the degree to which a solute is extracted in terms of the distribution coefficient of the solute between the two liquids. The distribution coefficient is defined as

$$
K_{D}=\frac{[\text { Solute }]_{\text {Organic }}}{[\text { Solute }]_{\text {Aqueous }}}
$$

where $[\text { Solute }]_{\text {Organic }}$ and $[\text { Solute }]_{\text {Aqueous }}$ are equilibrium concentrations of solute in the organic and aqueous phases. Coefficients of this type are a strong function of the degree of ionization of the solute. Non-ionized solutes tend to produce higher distribution coefficients into solvents with non-ionized extractants (non-ion exchange extractants) than into ionizing solutes since the extractant removes the neutral solute molecule rather than an individual ion, as in ion exchange.

A great deal of research has been done into the extraction of dilute acetic acid from aqueous waste or product streams. In industrial processes, the preferred method of removal for dilute concentrations of acetic acid is solvent extraction. [9] In many cases, the aqueous waste or product streams are contacted with an organic diluent to extract acetic acid. In some cases an extractant must be added to the diluent to aid in the extraction. These extractants form weak complexes with the solute to be extracted that are insoluble in water but are soluble in the organic phase. [17] Wardell and King (1978) determined the distribution coefficients displayed in Tables 2-2 and 2-3 for several types of organic solvents. Distribution coefficients were 
measured for acetic acid-water solutions only (no nitric acid present). Wardell and King (1978) state the equilibrium distribution coefficients of phosphoryl compounds as extractants in diluents follow the following trend phosphates $<$ phosphonate $<$ phosphine oxide. Data presented by Wardell and King (1978) examines the relationship between basicity, extractant concentration, and molecular weight for the distribution of carboxylic acids in various solvent systems. Some of the data indicate acetic acid distribution coefficients for trioctylamines as high as 9.9 while the distribution coefficient of tributyl phosphate (TBP) was found to be typically 2.3 at $100 \%$ TBP with no diluent. [18]

The degree of ionization for aqueous acetic acid solutions can be estimated as follows:

$$
K c=1.77 \mathrm{E}-5=10^{\left(-p K_{a}\right)}=\frac{\left[H^{+}\right]\left[A c^{-}\right]}{[H A c]}
$$

Or

$$
\frac{\left[A c^{-}\right]}{[H A C]}=\frac{1.77 \mathrm{E}-5}{\left[H^{+}\right]}
$$


Table 2-2: Ranges of Equilibrium Distribution Coefficients for Dilute Acetic Acid between Classes of Organic Solvents and Water. [16]

\begin{tabular}{|c|c|}
\hline Solvents & $\begin{array}{c}\text { Range of Distribution } \\
\text { Coefficients }\end{array}$ \\
\hline ethers $\left(\mathrm{C}_{4}-\mathrm{C}_{8}\right)$ & $0.63-0.14$ \\
\hline acetates $\left(\mathrm{C}_{4}-\mathrm{C}_{10}\right)$ & $0.89-0.17$ \\
\hline ketones $\left(\mathrm{C}_{4}-\mathrm{C}_{10}\right)$ & $1.20-0.61$ \\
\hline alcohols $\left(\mathrm{C}_{4}-\mathrm{C}_{8}\right)$ & $1.68-0.64$ \\
\hline
\end{tabular}

Table 2-3: Equilibrium Distribution Coefficients of Acetic Acid between Diluents and Water. [16] ${ }^{\text {a }}$ Chevron Solvent 25 is mostly C-8 and C-9 alkylated aromatics [17]

\begin{tabular}{|c|c|}
\hline Diluents & $\begin{array}{c}\text { Distribution } \\
\text { Coefficient }\end{array}$ \\
\hline Chevron Solvent 25 ${ }^{\mathrm{a}}$ & 0.009 \\
\hline Chloroform & 0.028 \\
\hline n-Hexanol & 0.88 \\
\hline n-Heptanol/n-Hexanol $(2: 1)$ & 0.30 \\
\hline Nitrobenzene & 0.06 \\
\hline n-Heptane & 0.02 \\
\hline
\end{tabular}


Thus, using a concentration of approximately $0.05 \mathrm{M}$ acetic acid, the percent ionized (degree of ionization multiplied by 100 ) is $1.86 \%$ in a solution of only water and acetic acid. With the addition of $0.5 \mathrm{M}$ nitric acid, the percent ionized decreased further. This also relates to the distribution coefficient since with nitric acid present, most acetic acid will be molecular and therefore more easily extracted into the organic phase. This does not mean that the increase in the distribution coefficient to the organic will be directly related to the fraction of acetic acid to the total acetic acid concentration, but it does give some insight into the distribution of acetic acid in aqueous solutions of interest. A possible contradicting effect is the presence of a mineral acid, if strong enough, could protonate the acetic acid and causes it to be in an ionic form, therefore suppressing extraction. [20]

In discussing potential solvent extraction options for this application, solvent options will be divided into two main groups, solvents that are partially miscible in aqueous solutions and solvents that are essentially immiscible in water. The first group includes the De Dietrich process which uses solvents such as ethyl acetate or methyl isobutyl ketone (MIBK) to remove acetic acid during manufacture of pharmaceutical products or cellulose acetate. Although this is a relatively mature technology, it is not likely to be attractive for removing acetic acid from nuclear spent fuel reprocessing streams due to the miscibility of the solvent that would leave some residual solvent after the acetate is removed. Thus, an additional process step is likely to be required to remove the remaining solvent. With a relatively volatile solvent, the additional removal step may be a reasonable choice, but in general, the use of miscible solvents makes solvent extraction much less advantageous. 
Immiscible solvents usually include a diluent and an active extractant, much like the systems used in fuel processing to remove actinides, fission products, and other metals. The diluent is likely to be a hydrocarbon such as kerosene or dodecane, and a variety of extractants can be used. The extractant, as well as the diluent, should be essentially insoluble in the aqueous phase. At least three extractants have been tested for removing acetic acid from aqueous phase, trioctylphosphine oxide (TOPO), tri-n-octylamine (TnOA), and tributylphosphate (TBP). The diluent only case was also considered.

The distribution coefficient using TBP is of particular interest for this investigation because TBP is already used as a solvent during the UREX, NPEX, and TRUEX segments of the UREX+ process. This makes it a leading candidate for use as an extractant with an appropriate organic diluent. TBP is a highly polar compound with its phosphoryl group acting as a Lewis base. This allows TBP to create an acid-base complex with acetic acid and gives a high equilibrium distribution coefficient. [20] The system used in the UREX+ process is a 30\% TBPdodecane organic solvent with acetic acid-nitric acid-water. Data has already been measured for $30 \%$ TBP-dodecane in a 1:1 organic to aqueous ratio with acetic acid-water. This data is shown in Figure 2-5 which shows as the initial concentration of the acid increases, the distribution coefficient decreases. [21] When a strong mineral acid is added to an acetic acid-water-TBP system, like nitric acid in this case, the acetic acid may be protonated and may be in an ionic state that will be difficult to extract to the organic stream. [20]

Much like TBP, tri-n-octyl amine (TnOA) will also have the same protonation result with the addition of a strong mineral acid to an acetic acid-water mixture. The distribution coefficient 
may also decrease because of competition of the strong mineral acid for TnOA since it has a higher affinity for the TnOA than its weaker acetic acid counterpart.

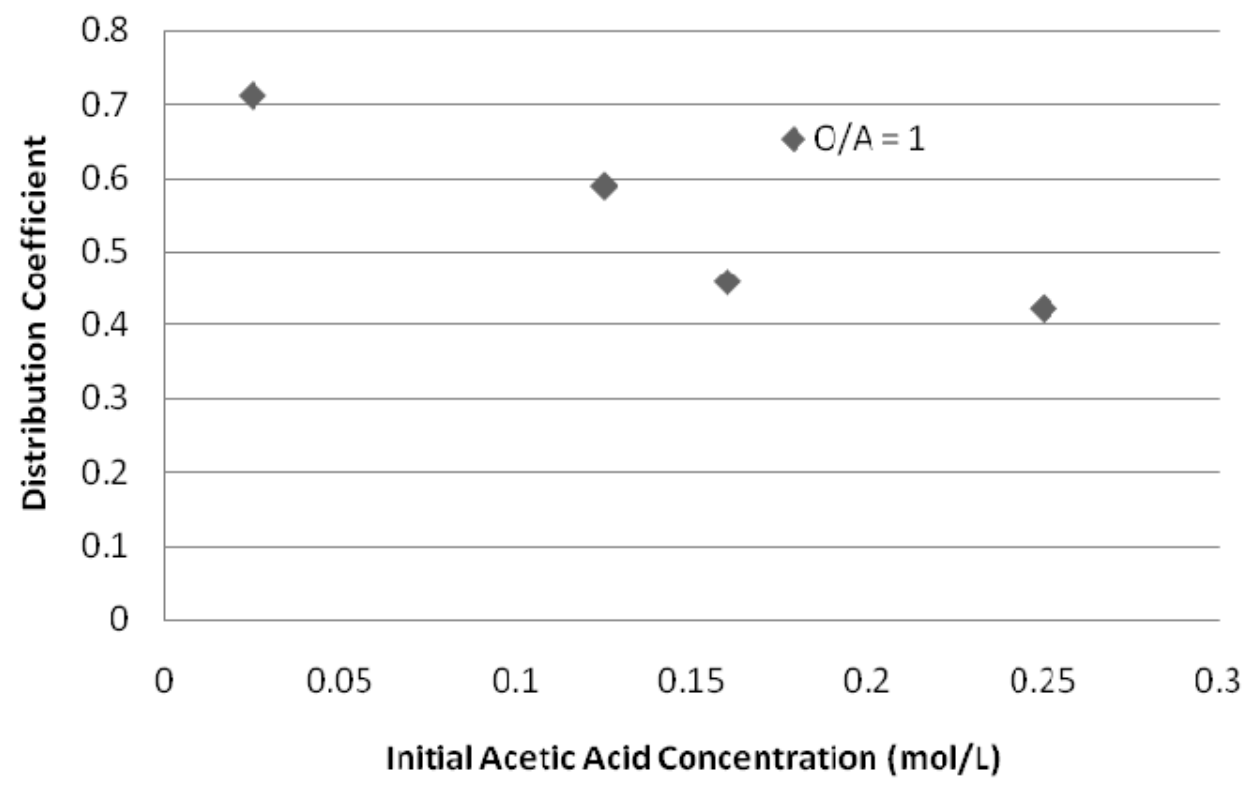

Figure 2-5. $K_{D}$ as a function of Acetic Acid Concentration using 30\% TBP-Dodecane Solvent with an Organic to Aqueous Ratio of 1. [21] 
Trioctylphosphine Oxide (TOPO) can be used in the extraction of acetic acid. TOPO's ability to extract acetic acid at various concentrations is shown in Figure 2-6. TOPO has been proposed as a possible extractant to be used in nuclear processes, however, the UREX+ processes do not include this solvent. Also, TOPO is a very expensive extractant when compared to TBP or TnOA. Therefore, TOPO will be used only if these other extractants prove ineffective. Another option that was considered was the diluent-only case. That is, the use of the diluent used throughout the fuel process system without the use of an extractant. Although something of a long-shot, this option can be evaluated experimentally very quickly. If one can use the same diluent as elsewhere in the fuel processing system, there would be minimal risk of undesirable effects from entrainment of diluent through the nitric acid product into other process steps. Also the diluent would not be likely to extract any metal ions (fission products, actinides, etc.), so the acetic acid should be relatively free of radioactivity. The principal path for radioactivity to reach the acetic acid probably would be via entrainment. Although, Table 2-3 shows that Chevron Solvent 25 does not appear to extract acetic acid alone, it possibly will extract some additional acetic acid in highly acidic solutions where a substantial portion of the acetic acid will not be ionized. There is also the rather remote possibility that other diluents such as dodecane will give somewhat higher distribution coefficients. However, the diluent-only option will be attractive only if the distribution coefficient for dodecane or a similar satisfactory diluent gives much higher distribution coefficients than that reported for Chevron Solvent 25. There is no hard limit for the value of the distribution coefficient that would be necessary for the diluent-only option to be viable, but we would like for the distribution coefficient to be 0.1 or larger. Smaller distribution coefficients require increasingly larger solvent flow rates and make operation of 


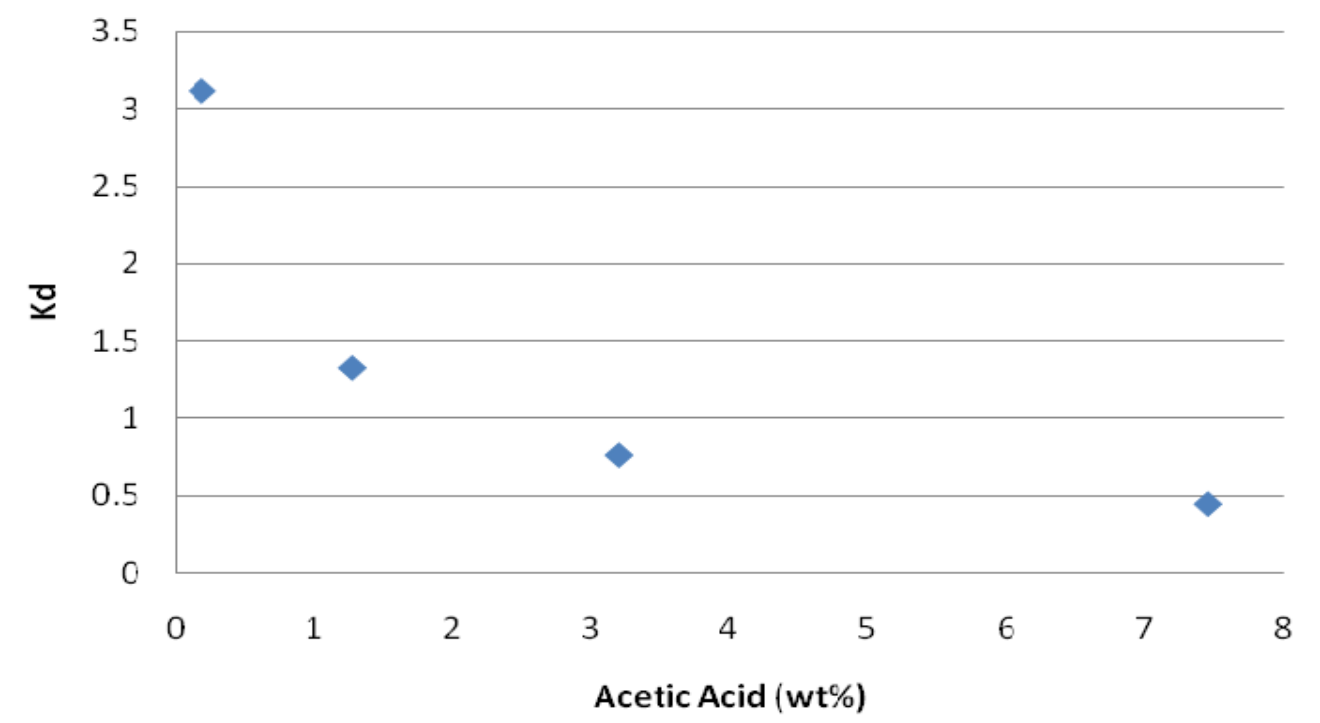

Figure 2-6. $K_{D}$ as a function of Acetic Acid wt\% using TOPO (22 wt \%) in Chevron Solvent

25. [17]

some liquid-liquid contactors difficult. Even if extraction could be practical with diluent-only, it will also be necessary to strip the acetic acid. The preferential approach would be to strip with water alone since the $\mathrm{pH}$ of the strip could be close to neutral, with hydrogen ion concentrations approaching values as low as $10^{-7}$ molar. However, if one wanted to improve the strip and/or concentrate the acetate, a slightly alkaline strip could be used. The best option for solvent extraction would be to use an immiscible solvent. If the diluent only case can yield sufficient extraction, it would be the most attractive approach. If not, the addition of TBP to any diluent would be second choice. The use of a TBP-dodecane system would be most favorable for this choice because of its expected use in UREX + processes. For any choice, the stripping of the acetic acid once it has been extracted into the organic will be needed. 


\subsection{Destruction}

This study assumed that there are limitations on the nature of reagents that can be added to the UREX+ process. So, there are limitations on how the acetic acid in the raffinate can be destroyed. Some methods thought to be potentially acceptable are Supercritical Water Oxidation, Wet-Air Oxidation, using a Corona Discharge Reactor, and using Hydrogen Peroxide. Other oxidation methods require adding a salt or other reagent that leaves a residue in the solution, and those residues may complicate downstream process steps and hinder reuse of the nitric acid, and/or could increase the amount of mass in the waste streams.

Using Supercritical Water Oxidation, under high temperature and pressure, the water molecule will become a non-polar solvent. This makes it a very good environment for oxidizing organic compounds. The process requires oxygen or hydrogen peroxide at temperatures higher than $647.3 \mathrm{~K}$ and pressures higher than $22.12 \mathrm{MPa}$ to maintain supercritical conditions. Either of these two oxidizing agents can completely breakdown the acetic acid into carbon dioxide and water. This process occurs in four main steps: (1) raising the pressure of the oxidizing agents, (2) the reaction, (3) separating the salt, and (4) depressurization and heat recovery. [22] While this method is expected to efficiently destroy acetic acid, the required high temperature and pressure conditions seem too extreme for a unit operation processing radioactive materials as part of the UREX+ process.

Consideration of wet-air oxidation to destroy acetic acid results in similar concerns as supercritical oxidation. A high temperature and a high pressure - though not as high as supercritical conditions - are required. This is so that the oxygen molecules are sufficiently reactive to interact with small carboxylic acids like acetic acid. The small size of the acetic acid 
molecule is unfavorable for oxidation under normal conditions. Also wet air oxidation is less efficient than supercritical oxidation at destroying acetic acid. A favorable approach to oxidize small acetic acid molecules may be to add a metal catalyst. Even so, there is no guarantee for complete oxidation of acetic acid unless the temperature is raised to $200^{\circ} \mathrm{C}$ or higher. [23]

Hydrogen Peroxide is a strong oxidant, and a mixture of ozone and hydrogen peroxide makes even stronger hydroxyl radicals. Hydroxyl radicals create acetate radicals from acetic acid. These radicals will be very active and will oxidize effectively to carbon dioxide and water. However, high temperatures - unfavorable to the UREX+ process - and a low $\mathrm{pH}$ are needed for this process to be able to operate optimally. [24]

Another possible destruction method is a corona discharge reactor. With this method, oxygen radicals can be produced from three different electron energy levels. Using this process with oxygen radicals and raising the $\mathrm{pH}$ will degrade the acetic acid to $75 \mathrm{ppm}$ from $100 \mathrm{ppm}$ at $\mathrm{pH} 1$ with the largest effect at $\mathrm{pH} 14$ where it is degraded down to $20 \mathrm{ppm}$. [25] A base would have to be added to raise the $\mathrm{pH}$ to 14 . Obviously a higher $\mathrm{pH}$ would require adding sodium ions or other neutralizing reagent, affect downstream processing, and makes nitric acid reuse impractical. The higher $\mathrm{pH}$ is also undesirable because plutonium is reduced at higher $\mathrm{pH}$ 's and may inappropriately precipitate from solution. Therefore, the corona discharge reactor is a nonviable option for acetic acid destruction in the UREX+ process.

Upon considering each of these destruction technologies, three constraints are considered: (1) nitric acid not being destroyed, (2) no new chemicals being added, and (3) no extreme equipment specifications for radioactive removal. It is desirable to recover and recycle 
nitric acid, so technology which destroys nitric acid or does not selectively destroy acetic acid over nitric acid is rejected. Likewise, no new chemicals are to be added without careful consideration. Also, the size of the equipment and pressure requirements of supercritical and wet air oxidation tend to cause these options to be severely discounted. No appropriate destruction technology is identified.

\section{RESULTS}

Each of the technologies discussed have some potential for the UREX+ process, but some prove to be more favorable than others. The potential advantages and disadvantages for these technologies are summarized in Table 2-4. Crystallization is a simple process but requires a preliminary concentration step to create conditions where the acetic acid will crystallize from solution; this technology would be considered if one needed to remove acetic acid from the recovered and concentrated nitric acid solution prior to its recycle. Distillation does not seem to 
Table 2-4. Technology Comparisons

\begin{tabular}{|l|l|l|}
\hline Technology & Pros & Cons \\
\hline Crystallization & $\begin{array}{l}\bullet \text { Used with distillation } \\
\text { Feasible } \\
\text { Favors acetic acid over } \\
\text { nitric acid }\end{array}$ & $\begin{array}{l}\bullet \text { Requires concentration } \\
\text { Some mechanical operation } \\
\text { needed }\end{array}$ \\
\hline Distillation & $\begin{array}{l}\text { Feasible } \\
\text { Used with Crystallization }\end{array}$ & $\begin{array}{l}\bullet \text { Removes water first which } \\
\text { concentrates nitric acid } \\
\text { Multiple towers required to } \\
\text { remove acetic acid }\end{array}$ \\
\hline Adsorption/Ion Exchange & $\begin{array}{l}\bullet \text { Uncertain Feasibility } \\
\text { Solvent Extraction }\end{array}$ & $\begin{array}{l}\bullet \text { No highly attractive } \\
\text { adsorbent found }\end{array}$ \\
\hline $\begin{array}{l}\text { Similar to other UREX+ } \\
\text { operations } \\
\text { diluent already in process } \\
\text { Data exist on similar } \\
\text { systems }\end{array}$ & $\begin{array}{l}\bullet \text { Restricted to solvents in } \\
\text { UREX+ } \\
\text { Strip step required } \\
\text { Possibly wash and/or solvent } \\
\text { scrub step required }\end{array}$ \\
\hline Destruction & $\begin{array}{l}\bullet \text { Could possibly eliminate } \\
\text { acetic acid } \\
\text { Creation of hydroxyl } \\
\text { radicals will completely } \\
\text { breakdown acetic acid }\end{array}$ & $\begin{array}{l}\bullet \text { Mainly need high } \\
\text { temperature and pressure } \\
\text { High pH needed for corona } \\
\text { discharge }\end{array}$ \\
\hline
\end{tabular}


offer any advantage for acetic acid removal due to the dilute concentration used unless coupled with crystallization or another unit operation. Distillation would require removal of most of the water before acetic acid is removed. Adsorption is a technically feasible option with similar concerns for additional process complexity as those for solvent extraction. Solvent extraction appears to be technically feasible, is a similar technology to that used elsewhere in the UREX+ system, and is a preferred method of removal for dilute acetic acid-water systems in industry; regeneration of the solvent and removal of the acetic acid adds complexity to the process however. No destruction technology for acetic acid in the UREX+ process was identified.

Overall, solvent extraction, probably using TBP extractant in dodecane (materials already used in $\mathrm{UREX}+$ ), appears to be the most promising approach to acetic acid removal. Distillation was not considered superior to solvent extraction except for higher concentrations of acetic acid than those expected in UREX+. However, if acetic acid removal can be delayed until the end of the UREX+ process when the nitric acid may be concentrated for recycle, distillation may remain an option, though not necessarily a better option than solvent extraction. Distillation to remove acetic acid selectively from nitric acid would be more complex than distillation simply to concentrate the nitric acid. As noted earlier, this would be a ternary distillation system that is expected to involve an acetic acid-nitric acid azeotrope. 


\section{CONCLUSIONS}

The primary method that seems the most feasible is solvent extraction, and the next efforts should focus on solvent extraction options. With no assurance that a practical solvent extraction technology can be found, other options should be considered if in the future solvent extraction proves to be less attractive. These alternate options are distillation, a combined distillation and crystallization, or carbon or polymer-based adsorption.

By careful selection of a solvent, acetic acid may be preferentially extracted from the aqueous phase. With the selection of the most promising technologies, further investigation through literature research and experimentation will lead to the most appropriate technology for the UREX+ process. The studies will need to cover the behavior of selected metal ions (metals used to simulate the fission products and actinides in the UREX raffinate) as well as acetic acid.

\section{ACKNOWLEDGEMENTS}

This work was supported by the U.S. Department of Energy, NERI program, under DOE Contract No. DE-PS07-05ID14713. 


\section{REFERENCES}

1. George F. Vandegrift, Monica C. Regalbuto, Scott B. Aase, Hassan A. Arafat, Allen J. Bakel, Delbert L. Bowers, James P. Byrnes, Mark A. Clark, Jeffery W. Emery, John R. Falkenberg, Artem V. Gelis, Lohman D. Hafenrichter, Ralph A. Leonard, Candido Pereira, Kevin J. Quigley, Yifen Tsai, Mark H. Vander Pol, and James J. Laidler. 227th ACS National Meeting, Anaheim, CA, 2004.

2. C. L. Riddle, J. D. Baker, J. D. Law, C. A. McGrath, D. H. Meikrantz,.Sol. Extr. \& Ion Exch.23, 449 (2005).

3. George F. Vandegrift (private communication) April 2007.

4. E.D. Collins, D.E. Benker, L.K. Felker, R.D. Taylor, G.D. Del Cul, B.B. Spencer, W.D. Bond,and D.O. Campbell presented at Proceedings of Global 2005 International Conference, American Nuclear Society 2005 (unpublished)

5. R.S. Barr and D.M.T. Newsham. Chem. Eng. J., 33, 791986.

6. W.F. Linke and A. Seidell. Solubilities: Inorganic and Organic Compounds. $4^{\text {th }}$ ed. (Washington D.C., 1965) p.1128,1132.

7. K. Nagahama and T.X. Jiang. Fluid Phase Equil. 53, 261 (1989)

8. D. J. Shah and K. K. Tiwari. J. Sep. Proc. Tech. 2 , 1 (1981)

9. H. Cheung, R. S. Tanke, and G. P. Torrence Ullmann's Encyclopedia of Industrial Chemistry (Wiley-VCH, 2003) $6^{\text {th }}$ ed. Vol.1 p. 149-178.

10. Zhigang Lei, Chengyue Li, Yingxia Li, and Biaohua Chen. Sep. \& Pur. Tech. $\underline{36}$, 2004 (2004). 
11. S. K. Ganguly and A. N. Goswami. Sep. Sci. \& Tech. $\underline{31,} 1267$ (1996)

12. Yue Kuo, C.L. Munson, W.G. Rixey, A.A. Garcia, Mathew Frierman, and C.J. King. Sep. \& Pur. Meth. $\underline{16}, 31$ (1987)

13. Ikuo Abe, Katsuni Hayashi, Mutsuo Kitagawa, and Toshihiro Urahata. Bul. Chem. Soc. Jap. $\underline{52}, 1899(1979)$

14. Motoyuki Suzuki and Takao Fujii. AIChE J. 28, 380 (1982).

15. Shie W. Wang, Anthony L. Hines, and Emanual Pedram. Ind. Eng. Chem. Fund. 20, 350 (1981)

16. Mathew Frierman, Yue Kuo, Dilip Joshi, A. A. Garcia, and C. J. King. Sep. \& Pur. Meth. 16 91 (1987).

17. G. Maurer and A. Schunk. Fluid Phase Equil. 239, 223 (2006)

18. James M. Wardell and C. Judson King. J. Chem. Eng. Data 23, 144 (1978)

19. N.L. Ricker, J.N. Michaels, and C.J. King. J. Sep. Proc. Tech. 1 , 36 (1979)

20. M.N. Ingale, and V.V. Mahajani. Sep. Tech. $\underline{6}, 1$ (1996)

21. B.C. Roy, M.J. Alam, M. Goto. J. Applied Sci. $\underline{6}, 1$ (2006)

22. M. D. Bermejo, and M. J. Cocero. AIChE J. $\underline{52}, 3933$ (2006)

23. Vedprakash S. Mishra, Vijaykumar V. Mahajani, and Jyeshtharaj B. Joshi. Ind. \& Eng. Chem. Res. 34, 2 (1995).

24. E. Neyens and J. Baeyens. J. Haz. Mat. $\underline{98}, 33$ (2003).

25. Noraki Sano, Tatsuya Fujimoto, Toru Kawashima, and Tatsuo Kanki. Proceedings of the Asia-Pacific Conference p. 89 (2005). 


\section{CHAPTER III: Extracting Acetic Acid from Acidic Solutions}

Chapter III is a slightly revised version of an article by the same name originally published in the journal Separation Science and Technology, 43 (9-10), pp 2537-2547 (2008) in 2008 by Jessica Mitchell, Jared Johnson, Robert M. Counce, Jack S. Watson, Barry B. Spencer, and G.D. Del Cul: 


\begin{abstract}
In the UREX+ process, acetic acid must be removed from the raffinate stream to avoid interference with the recovery and recycle of nitric acid solutions. Solvent extraction was selected to be the most promising approach to accomplish this cleanup. Acetic acid partitioning into pure diluents used in the UREX+ process were found to be too low for an effective separation. Of the solvents tested, the most promising solvents for the extraction of acetic acid were found to be TBP in dodecane and TBP in FS-13.
\end{abstract}

\title{
1. INTRODUCTION/BACKGROUND
}

The UREX+ process is a proposed new approach to separating the components of spent nuclear fuels. It has 5 steps all utilizing solvents to extract various components of the spent fuel. The current steps considered for the UREX+ process are shown in Figure 3-1. The first step is called the UREX step and uses tributylphosphate (TBP) with dodecane and acetohydroxamic acid to selectively extract uranium and technetium. The purpose of the acetohydroxamic acid is to prevent the extraction of plutonium in the UREX step. The next step uses polyethylene glycol (PEG) with phenyltrifluoromethyl sulfone, FS-13, and cobalt dicarbollide to extract both strontium and cesium. An alternative to CCD-PEG step is the FPEX step uses a BOB Calix solvent in order to extract Sr and Cs. The NPEX step is next and uses the same TBP-dodecane solvent system as UREX without the acetohydroxamic acid so that plutonium and neptunium are co-extracted with any remaining uranium. During the TRUEX segment of the process, americium, curium, the rare earth elements(lanthanides), and any remaining plutonium and 
neptunium are extracted by a solvent containing octyl(phenyl)-N, N-di-isobutyl

carbamoylmethyl phosphine oxide (CMPO) and TBP with $n$-dodecane. The final step is the

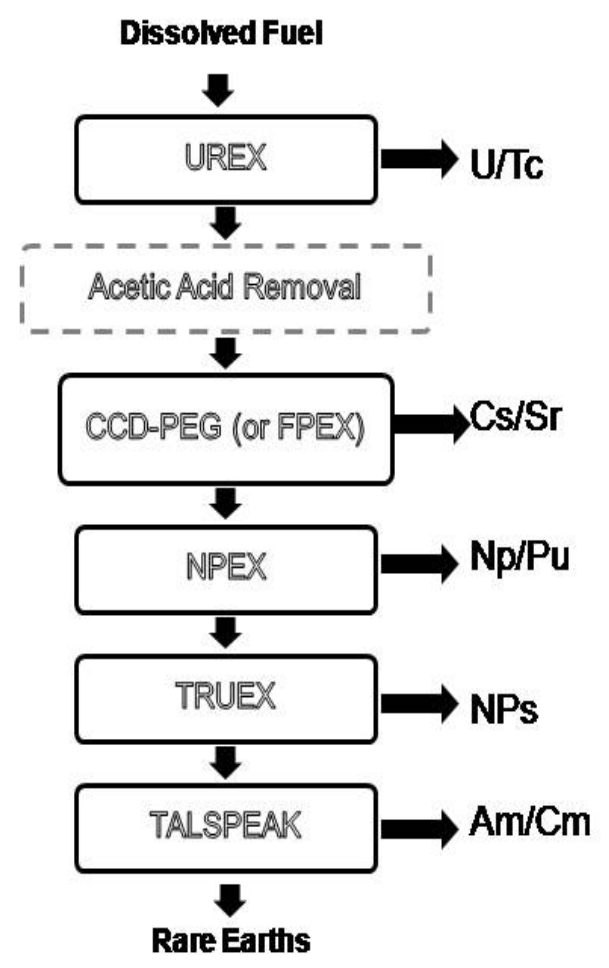

Figure 3-1. UREX+ Flowsheet with Proposed Acetic Acid Removal Step 
TALSPEAK step that extracts the lanthanides from the TRUEX product using diethylene triamine pentaacetic acid (DTPA) and a complexing agent (e.g. citric or lactic acid) to hold the actinides (e.g. americium and curium) in the aqueous solution.[1,2].

In the first step of the UREX+ process, acetohydroxamic acid is added to suppress extraction of plutonium when TBP-dodecane extracts uranium and technetium. Plutonium forms a complex with acetohydroxamic acid so that TBP is unable to extract it from aqueous solution. After uranium and technetium are extracted, this complexing agent hydrolyzes to form acetic acid and hydroxylamine. While the hydroxylamine will break down in strong acid solutions and/or at elevated temperatures, the acetic acid degradation product needs to be removed or destroyed so it will not interfere in the recycling of nitric acid [2]. The acetic acid removal is shown in Figure 3-1 occurring just after the UREX step. The optimum location of the acetic acid removal step is not established, but it should be used after the acetohydroxamic acid has decomposed. The extraction of acetic acid is also needed for many non-nuclear manufacturing processes, and the results from this study could also be useful to those processes.

\subsection{Alternate Separation Methods Considered}

Many technologies were examined as possible acetate removal and/or destruction methods, but some proved more favorable than others for the UREX+ process. Technologies such as membrane separation and ion exchange were eliminated early in the literature review. No membrane was found that was sufficiently selective to remove acetic acid effectively in a single pass, and multistage membrane operations did not appear attractive. Ion exchange was eliminated because no ion exchange material was identified with sufficient selectivity for acetate 
ions over higher concentrations of nitrate ions in a mixed stream. Crystallization is a simple process but requires a preliminary concentration step to create conditions where the acetic acid could crystallize from solution [5-7]. Distillation does not seem to offer any advantage for acetic acid removal unless coupled with crystallization or another unit operation [6]. Adsorption was considered, but no adsorbent was found with sufficient selectivity for acetic acid [3,4]. Destruction methods could completely destroy the acetic acid. However, the extreme conditions required to destroy acetic acid are likely to destroy all or most of the nitric acid as well [8-10].

It was decided that solvent extraction would be the most promising method to pursue further. Solvent extraction appears to be technically feasible and is a similar technology to that used elsewhere in the UREX+ system. A few solvent mixtures (diluent and extractant) have been shown to remove acetic acid from nitric acid [11,12], however, these mixtures are also capable of extracting some of the radioactive components in spent fuel solutions and add unwanted complexity to the process being developed. In addition, there appeared to be further opportunities for considering alternative solvents. The focus of this study is to identify an agent capable of extracting acetic acid without co-extraction of other radioactive components, or with

minimal extraction of radioactive components. Ideally, the solvent selected would be commonly used in the UREX+ process.

\subsection{Solvent Selection}

The three solvents/diluents discussed, dodecane, dichloroethane, and FS-13 were chosen because dodecane is already present in the UREX+ process and dichloroethane is comparable in some ways to phenyltrifluoromethyl sulfone, also known as FS-13 solvent, utilized in the PEGCCD step [13]. Dodecane is used in the UREX, NPEX, and TRUEX steps of the process, and 
FS-13 solvent is used during the PEG/CCD step. It was decided that the most probable place to extract the excess acetic acid was after the UREX step and before the PEG/CCD step (Figure 31). At this point, the acetohydroxamic acid is hydrolyzed into acetic acid and hydroxylamine nitrate (HAN). Since FS-13 was not readily available early in this study, dichloroethane was used initially as an analogue for FS-13 solvent [13]. Dichloroethane is not a suitable solvent for the UREX+ process.A limited quantify of FS-13 became available during the experimental trials and was also tested. These solvents were also used in the experiment with tributyl phosphate (TBP) at a $2.5 \mathrm{M}$ concentration.

\section{EXPERIMENT}

The distribution coefficients for a number of solvents and conditions were measured to determine the amount of acid transferred into the organic phase from solutions similar to those found in the UREX + process. Solutions of various acidic concentrations were prepared ranging from $0.01 \mathrm{M}$ to $1 \mathrm{M}$ of both nitric and acetic acids to serve as the reference aqueous solutions for this study. Organic solvents (diluents) dichloroethane, dodecane, and FS-13 were the first solvents tested. Dodecane 99\% was obtained from ACROS Chemicals, ACS grade dichloroethane was obtained from Fisher Scientific, and FS-13 was obtained from Marshallton Reasearch Laboratories. Using a $250 \mathrm{~mL}$ separatory funnel, $10 \mathrm{~mL}$ of aqueous and $10 \mathrm{~mL}$ of organic solvent were added. The funnel was hand-shaken for approximately one minute and settling was allowed for full separation of the layers. The aqueous layer was drawn off into a small beaker and analyzed using a Mettler-Toledo SevenEasy pH meter and a Brinkmann 765 Dosimat set on dose mode containing $1 \mathrm{M}$ caustic solution. Using the amount of caustic required 
to obtain the equivalence point of the aqueous solution, the concentration of acid in the aqueous phase was determined by equation 1 .

$$
[A Q]=\frac{[\text { Base }] \times V_{\text {Base }}}{\left(V_{\text {Sample }}\right)}
$$

[Base] represents the standardized concentration of the solution used to titrate, $V_{\text {Base }}$ is the amount of base needed to reach the equivalence point, $V_{\text {Sample }}$ is the sample size, and [AQ] is the calculated concentration of the aqueous phase after mixing with the organic phase. The amount of acid in the organic phase is then found by mass balance.

$$
\left[A Q_{\text {Original }}\right] V_{\text {Original }}-[A Q] V_{\text {sample }}=[O R G] V_{\text {ORG }}
$$

[AQ Original] represents the initial concentration of the aqueous phase when put into the separatory funnel. $V_{\text {Original }}$ is the volume put into the separatory funnel. [ORG] is the concentration of acid in the organic phase calculated by the difference of the initial aqueous acid concentration and aqueous concentration after equilibration, and $\mathrm{V}_{\mathrm{ORG}}$ is the volume of organic initially in the separatory funnel. The distribution coefficient was then calculated using equation 3.

$$
K_{D}=\frac{[O R G]}{[A Q]}
$$

When using a mixture of nitric and acetic acids with equal volumes, the amount of caustic required to reach equivalence must be read from the titration plot with two equivalence points and calculated as shown in equations 4 and 5 and Figure 3-2.

$$
V_{2}-V_{1}=V_{\text {Base-Acetic }}
$$




$$
V_{1}=V_{\text {Base-Nitric }}
$$

$V_{2}$ is the second equivalence point and $V_{1}$ is the first equivalence point. The calculations result in the volume of base required to reach the end point for equal volume acetic and nitric acids, respectively.

\section{RESULTS AND DISCUSSION}

\subsection{Titration Curve Analysis}

For the single acid case, the procedure is simple. The steepest point of the curve where the concavity changes is the equivalence point reading (Figure 3-2). The volume of base required is then simply read from the $\mathrm{x}$-axis. Using this value, the above calculations can be completed.

For the mixed acid case, the procedure becomes slightly more complicated. The titration curve is similar to that of a diprotic acid analysis with two equivalence points. In the case of nitric and acetic acids, the first curve is completion of nitric acid neutralization while the difference between the endpoints of the first curve and the second curve is the completion of acetic acid neutralization (Figure 3-3) [14,15]. Using equations 4 and 5, the volume of base needed to neutralize each acid can be found and then inserted into equations 1-3 to obtain a distribution coefficient for each acid.

\subsection{Acetic Acid Extraction from Water-Acetic Acid Solutions}

Tests were run to determine the distribution coefficient for acetic acid between aqueous and diluent phases (Table 3-1). The distribution coefficients found in this study for dodecane was 
small, but generally comparable to those reported by Judd King using several diluents with acetic acid (Table 3-2). The distribution for the other two solvents, dichloroethane and FS-13 were also small. A plausible explanation of the significant negative numbers for FS-13 is that there may 


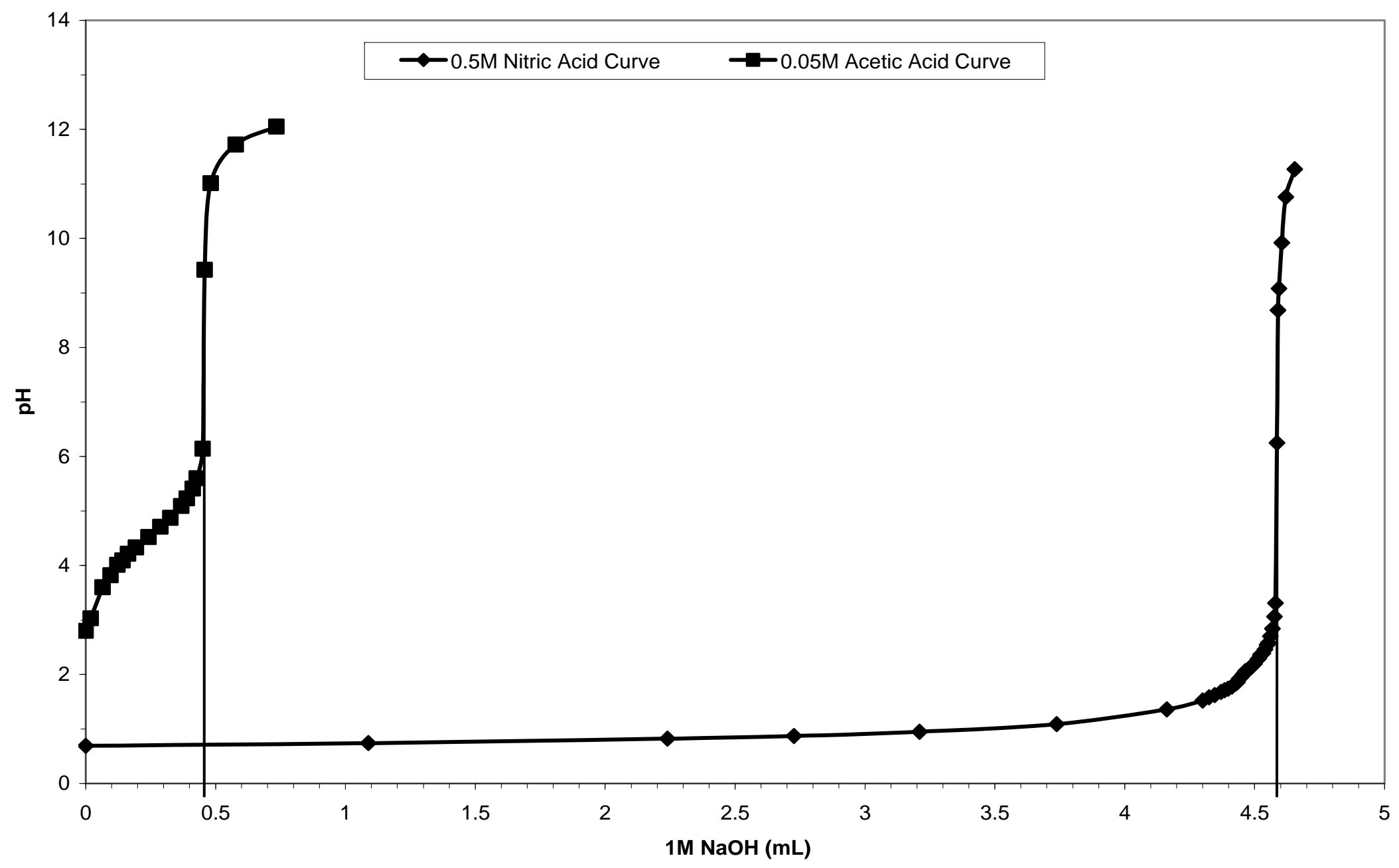

Figure 3-2. Single Acid Titration Curves (Nitric Acid and Acetic Acid) 


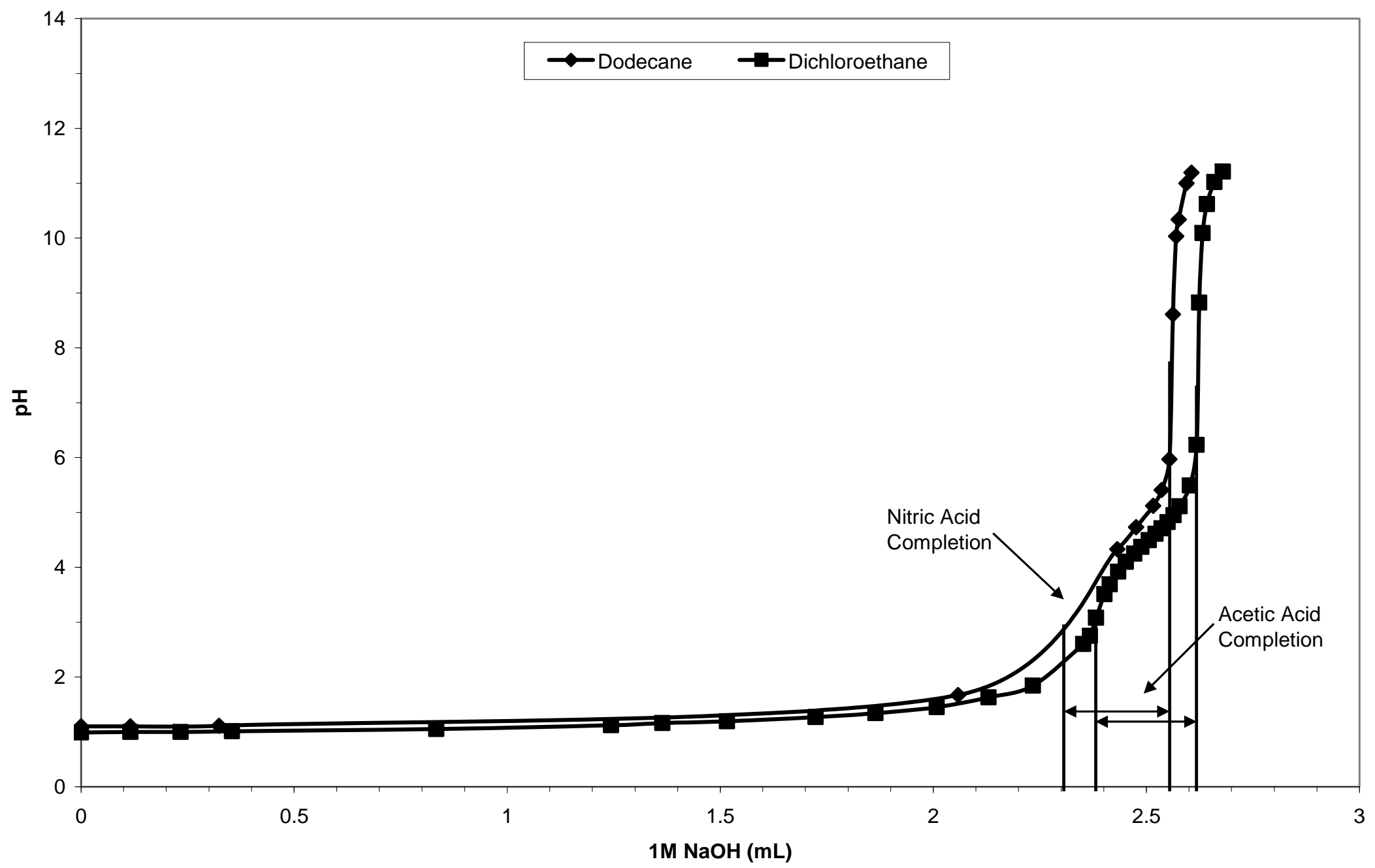

Figure 3-3. Mixed Acid Titration Curves (Both Nitric and Acetic Acid Together). 
Table 3-1. Extraction of Acetic Acid in Experimental Organic Diluents.

\begin{tabular}{|c|c|c|}
\hline $\begin{array}{l}\text { Acetic Acid } \\
\text { Concentration }\end{array}$ & $\mathrm{K}_{\mathrm{D}}$ & $\begin{array}{c}\text { Standard } \\
\text { Deviation }\end{array}$ \\
\hline \multicolumn{3}{|c|}{ Dodecane } \\
\hline $0.01 \mathrm{M}$ & -0.00397 & 0.022258 \\
\hline $0.05 \mathrm{M}$ & 0.006039 & 0.003788 \\
\hline $0.5 \mathrm{M}$ & 0.050085 & 0.01119 \\
\hline $1 \mathrm{M}$ & 0.010973 & 0.034771 \\
\hline \multicolumn{3}{|c|}{ Dichloroethane } \\
\hline $0.01 \mathrm{M}$ & 0.041742 & 0.020909 \\
\hline $0.05 \mathrm{M}$ & 0.01898 & 0.008563 \\
\hline $0.5 \mathrm{M}$ & 0.030064 & 0.024639 \\
\hline $1 \mathrm{M}$ & 0.050004 & 0.01795 \\
\hline \multicolumn{3}{|c|}{ FS-13 } \\
\hline $0.01 \mathrm{M}$ & -0.83036 & 0.00089 \\
\hline $0.05 \mathrm{M}$ & -0.25364 & 0.2257 \\
\hline $0.5 \mathrm{M}$ & -0.0696 & 0.04424 \\
\hline $1 \mathrm{M}$ & -0.00995 & 0.023845 \\
\hline
\end{tabular}


Table 3-2. Extraction of Acetic Acid in Pure Organic Diluents.

\begin{tabular}{lcc}
\hline Diluent & $\mathrm{K}_{\mathrm{D}}$ & Reference \\
\hline Chevron Solvent 25 & 0.009 & {$[3]$} \\
n-Hexanol & 0.88 & {$[3]$} \\
Nitrobenzene & 0.06 & {$[3]$} \\
Chloroform & 0.028 & {$[3]$} \\
n-Heptane & 0.02 & {$[3]$} \\
\hline
\end{tabular}

have been some transfer of an acidic contaminant from the organic phase to the aqueous phase.

\subsection{Nitric Acid Extraction from Water-Nitric Acid Solutions}

The next tests involved determining the distribution coefficient for nitric acid between aqueous and diluent phases. When contacting aqueous nitric acid with the three diluents tested, a low distribution coefficient was obtained similar to those measured with acetic acid solutions (Table 3-3). This was expected since nitric acid almost completely dissociates in water.

\subsection{Nitric and Acetic Acid Extraction from Water-Acetic Acid-Nitric Acid Solutions}

A two equivalence point titration curve was produced using both nitric and acetic acids in the aqueous layer. (Figure 3-3) The distribution coefficients of acetic acid and nitric acid separately both yield little extraction. When combined, the distribution coefficients of acetic and nitric acid are still very small (See Table 3-4). As shown in Table 3-4, n-dodecane extracts nitric acid at a higher ratio than acetic acid. With dichloroethane, acetic extracts more strongly than nitric acid. The negative extraction values probably mean the very small extent of extraction is within the experimental error. This case is represented by the larger negative values in the FS-13 results. In 
either case, all the distribution coefficients show that there is a need for another approach to the extraction of acetic acid.

\subsection{5M Tributyl Phosphate Mixed With Organic Diluents}

The addition of tributyl phosphate (TBP) to the system can be used to enhance the extraction of either acid when using Chevron Solvent 25 [11]. Addition of tributyl phosphate to these diluents also improves the extraction of both acetic and nitric acids as shown in Table 3-5. Tests were made with aqueous solutions of $0.05 \mathrm{M}$ acetic acid only and $0.5 \mathrm{M}$ nitric acid only. Then an equalvolume mixture of $0.05 \mathrm{M}$ acetic acid and $0.5 \mathrm{M}$ nitric acid was tested. While the TBP in 
Table 3-3. Extraction of Nitric Acid in Pure Organic Diluents.

\begin{tabular}{|c|c|c|}
\hline $\begin{array}{c}\text { Nitric Acid } \\
\text { Concentration }\end{array}$ & $\mathrm{K}_{\mathrm{D}}$ & $\begin{array}{c}\text { Standard } \\
\text { Deviation }\end{array}$ \\
\hline \multicolumn{3}{|c|}{ Dodecane } \\
\hline $0.01 \mathrm{M}$ & 0.050017 & 0.022206 \\
\hline $0.05 \mathrm{M}$ & 0.061638 & 0.050059 \\
\hline $0.5 \mathrm{M}$ & 0.027958 & 0.00172 \\
\hline $1 \mathrm{M}$ & -0.00474 & 0.009551 \\
\hline \multicolumn{3}{|c|}{ Dichloroethane } \\
\hline $0.01 \mathrm{M}$ & 0.020709 & 0.032938 \\
\hline $0.05 \mathrm{M}$ & 0.020515 & 0.016487 \\
\hline $0.5 \mathrm{M}$ & -0.02579 & 0.030955 \\
\hline $1 \mathrm{M}$ & 0.015247 & 0.002282 \\
\hline \multicolumn{3}{|c|}{ FS-13 } \\
\hline $0.01 \mathrm{M}$ & -0.83136 & 0.00016 \\
\hline $0.05 \mathrm{M}$ & -0.51616 & 0.021645 \\
\hline $0.5 \mathrm{M}$ & -0.10366 & 0.018025 \\
\hline $1 \mathrm{M}$ & -0.03877 & 0.048026 \\
\hline
\end{tabular}


Table 3-4. Extraction of Acetic and Nitric Acids in Organic Diluents.

\begin{tabular}{|c|c|c|c|c|}
\hline $\begin{array}{c}\text { Ratio of } \\
\text { Concentrations }\end{array}$ & \multicolumn{2}{|c|}{ Nitric Acid } & \multicolumn{2}{|c|}{ Acetic Acid } \\
\hline (Acetic:Nitric) & $\mathrm{K}_{\mathrm{D}}$ & $\begin{array}{l}\text { Standard } \\
\text { Deviation }\end{array}$ & $\mathrm{K}_{\mathrm{D}}$ & $\begin{array}{l}\text { Standard } \\
\text { Deviation }\end{array}$ \\
\hline \multicolumn{5}{|c|}{ Dodecane } \\
\hline $0.5: 0.025$ & 0.030 & 0.012 & -0.015 & 0.003 \\
\hline $0.5: 0.25$ & 0.026 & 0.024 & -0.001 & 0.001 \\
\hline $0.5: 0.5$ & 0.008 & 0.010 & -0.022 & 0.003 \\
\hline $0.25: 0.5$ & -0.003 & 0.001 & -0.014 & 0.005 \\
\hline $0.025: 0.5$ & -0.011 & 0.000 & -0.081 & 0.004 \\
\hline \multicolumn{5}{|c|}{ Dichloroethane } \\
\hline $0.5: 0.025$ & 0.058 & 0.098 & 0.096 & 0.036 \\
\hline $0.5: 0.25$ & 0.024 & 0.017 & 0.048 & 0.012 \\
\hline $0.5: 0.5$ & 0.021 & 0.002 & 0.096 & 0.029 \\
\hline $0.25: 0.5$ & 0.013 & 0.012 & 0.078 & 0.029 \\
\hline $0.025: 0.5$ & 0.037 & 0.007 & 0.061 & 0.006 \\
\hline \multicolumn{5}{|c|}{ FS-13 } \\
\hline $0.5: 0.025$ & -0.588 & 0.010 & -0.010 & 0.002 \\
\hline $0.5: 0.25$ & -0.200 & 0.079 & 0.048 & 0.047 \\
\hline $0.5: 0.5$ & -0.048 & 0.022 & -0.038 & 0.016 \\
\hline $0.25: 0.5$ & -0.065 & 0.024 & -0.014 & 0.001 \\
\hline $0.025: 0.5$ & 0.060 & 0.010 & -0.306 & 0.006 \\
\hline
\end{tabular}


Table 3-5. Extraction of Acids in 2.5M TBP Mixtures

\begin{tabular}{|c|c|c|c|c|}
\hline \multirow[b]{2}{*}{ Concentration of Acid } & \multicolumn{2}{|c|}{ Nitric Acid } & \multicolumn{2}{|c|}{ Acetic Acid } \\
\hline & $\mathrm{K}$ & $\begin{array}{l}\text { Standard } \\
\text { Deviation }\end{array}$ & $\mathrm{K}$ & $\begin{array}{l}\text { Standard } \\
\text { Deviation }\end{array}$ \\
\hline \multicolumn{5}{|l|}{ Dodecane } \\
\hline 0.05M Acetic Acid & --- & --- & 1.408 & 0.021 \\
\hline 0.5M Nitric Acid & 0.404 & 0.073 & --- & --- \\
\hline 0.025M:0.25M Mixture & 0.308 & 0.081 & 1.481 & 0.161 \\
\hline \multicolumn{5}{|l|}{ Dichloroethane } \\
\hline 0.05M Acetic Acid & --- & --- & 1.255 & 0.010 \\
\hline 0.5M Nitric Acid & 0.193 & 0.021 & --- & --- \\
\hline 0.025M:0.25M Mixture & 0.134 & 0.012 & 1.350 & 0.124 \\
\hline \multicolumn{5}{|l|}{ FS-13 } \\
\hline 0.05M Acetic Acid & --- & --- & 0.530 & 0.063 \\
\hline 0.5M Nitric Acid & 0.259 & 0.014 & --- & --- \\
\hline 0.025M:0.25M Mixture & 0.133 & 0.012 & 1.083 & 0.044 \\
\hline
\end{tabular}


dodecane has the largest distribution coefficient for acetic acid out of the three diluents, the solvent with dichloroethane has the largest separation factor. The separation factor is the ratio of acetic acid to nitric acid distribution coefficients in the mixtures. FS-13 also shows a high separation factor and might be suitable in the UREX+ process since, as noted earlier, dichloroethane is unsuitable. FS-13 also shows the highest jump between the distribution coefficients of the single acids and the mixture. This is favorable for the UREX+ process and will be explored in further research as a method to extract acetic acid from the waste stream.

\section{CONCLUSIONS}

The diluent results confirm the results by King and coworkers [3] that neither acetic acid nor nitric acid is extracted effectively by simple hydrocarbon solvents. All of the solvents and extractants used by King were partially soluble in water or able to extract salts, including radioactive compounds present in the UREX+ process. FS-13 is not soluble in water and is already used in one proposed step of the UREX + process. The most promising solvents for the extraction of acetic acid are TBP in dodecane and TBP in FS-13. Each solvent system exhibits a distribution coefficient greater than one with a sufficiently large separation factor.

\section{ACKNOWLEDGEMENTS}

The authors are grateful to Dean Peterman, Joseph Birdwell, George Vandegrift, Catherine Mattus, and Matthäus Siebenhofer for their support and advice during the course of 
this work. This work was supported by the U.S. Department of Energy, NERI program, under DOE Contract No. DE-PS07-05ID14713.

\section{REFERENCES}

1. George F. Vandegrift, Monica C. Regalbuto, Scott B. Aase, Allen J. Bakel, Terry J. Battisti, Delbert Bowers, James P. Byrnes, Mark A. Clark, Jeff W. Emery, John R. Falkenberg, Artem V. Gelis, Candido Pereira, Lohman Hafenrichter, Yifen Tsai, Kevin J. Quigley, and Mark H. Vanderpol. Proceedings of ATLANTE 2004, Nimes, France, 2004.

2. George F. Vandegrift. Email Communication. April 2007.

3. Y. Kuo, C. L. Munson, W. G. Rixey, A. A. Garcia, M. Frierman and C. J. King, Sep. \& Pur. Meth. 16, 31 (1987)

4. M. Frierman, Y. Kuo, D. Joshi, A. A. Garcia and C. J. King, Sep. \& Pur. Meth., 18, 91 (1987)

5. W. F. Linke, A. Seidell. Solubilities, Inorganic and Metal-Organic Compounds, Vol II, $4^{\text {th }}$ edn.; American Chemical Society; Washington D.C., 1965.

6. K. Nagahama and T. X Jiang, Fluid Phase Equil., 53, 261 (1989).

7. A.S. Narayana, S. C. Nalk, and P. Rath, J. Chem. Eng. Data, 30, 483 (1985).

8. M.D. Bermejo and M.J. Cocero, AIChE J., 52, 3933 (2006). 
9. Noriaki Sano, Tatsuya Fujimoto, Toru Kawashima, and Tatsuo Kanki. Sustainable Energy and Environmental Technologies, Proceedings of the Asia-Pacific Conference 89 (2001).

10. Vedprakash S. Mishra, Vijaykumar V. Mahajani, and Jyeshtharji B. Joshi. Ind. \& Chem. Eng. Res. $\underline{34} 2$ (1995).

11. N.L. Ricker, J.N. Michaels, C.J. King. Sep. Proc. Tech. 136 (1979).

12. James M. Wardell, C.J. King, J. Chem. Eng. Data. 23, 144(1978).

13. E.S. Stoyanov, I.V. Smirnov, V.A. Babain, N.G. Antonov, D. Peterman, R.S. Herbst, T.A. Todd, and T.A. Luther Radiochem. 46, 587 (2004).

14. Catherine Mattus, Email Communication, June 2007.

15. Douglas A. Skoog, Donald M. West, F. James Holler, and Stanley R. Crouch, Analytical Chemistry: An Introduction, $7^{\text {th }}$ Ed.; Thomson Learning, Inc.:U.S.A. (2000). 


\section{CHAPTER IV: PLACING THE ACETIC ACID STEP INTO THE UREX+ PROCESS}

Chapter IV of this report is taken from the Master's Thesis of Jessica A. Mitchel, Removing Acetic Acid from the UREX+ Process, University of Tennessee (2008) 


\begin{abstract}
In the UREX+ process, acetohydroxamic acid is used to suppress plutonium extraction in the first process step (known as the UREX step). However, acetohydroxamic acid hydrolyzes during the UREX+ process into acetic acid and hydroxyl amine nitrate. Solvent extraction was determined to be the most suitable method for removing acetic acid from the UREX+ process streams. Experimental data have shown sufficient extraction values for acetic acid into the solvent system of tributyl phosphate and dodecane and favorable separation factors between acetic acid and nitric acid in this solvent system. Using this experimental data, worst-case estimates of acetic acid concentration, and literature information on each of the steps of the UREX+ process, it was determined acetic acid will not cause a problem in the UREX+ process. A process flow sheet is proposed for the selective removal of acetic acid.
\end{abstract}

\title{
1. Introduction
}

Acetic acid is a degradation product of acetohydroxamic acid (AHA) that is used in the UREX process to suppress plutonium extraction. The acetic acid must eventually be removed from the UREX+ process stream to purge acetic acid and recycle nitric acid. Previous work done by these authors has shown that solvent extraction using a TBP-dodecane organic solvent system is suitable to remove this acid from an aqueous nitric acid stream. [1] Since similar solvent mixtures are used throughout the $\mathrm{UREX}^{+}$process, some acetic acid is expected to be extracted as long as acetic acid is present in the aqueous stream. The purpose of this paper is to 
estimate any likely deleterious effects of acetic acid on the process steps of the UREX+ process and suggest the most appropriate location of the acetic acid removal step. Each process step within the process will be examined, and data obtained in this activity on acetic acid extraction will be used to estimate the concentrations of acetic acid in each raffinate and extractant stream. This allows determination of the fate of the acetic acid if it is introduced to each process step via the aqueous stream and the potential for problems from acetic acid presence. This extraction provides insight into the potential that acetic acid extraction could affect the extraction of desired components in each step of the UREX+ process. The easiest placement of the acetic acid removal step would be at the end of the process steps just before the nitric acid is concentrated for recycle. That would be after the more valuable components have been removed. In that case, there would be fewer components present with the acetic acid removed to complicate its disposal. This paper investigates the affect that acetic acid has on the extraction of desired components in any of the UREX+ process steps. This work is necessary to determine if acetic acid requires earlier removal, before any affected process step.

\section{Background}

\subsection{UREX+ Process}

The UREX+ process used in this study is shown in figure 4-1. The UREX+ process has been under development for many years, and throughout those years, many flow sheets have developed. For this study, the flow sheet presented in figure 4-1 is being used, a slightly modified UREX +3 flow sheet to include two replacement possibilities - TALSPEAK in place of Cyanex-301 and FPEX as a possible alternative to CCD-PEG. 
During the UREX step, uranium and technetium are extracted and purified using a TBPdodecane solvent system. Plutonium extraction is suppressed in this step by adding AHA to the aqueous feed. AHA is hydrolyzed slowly under the acid conditions of the aqueous phase. CCDPEG performance is not affected significantly by AHA. [2] However, if the second step is FPEX,

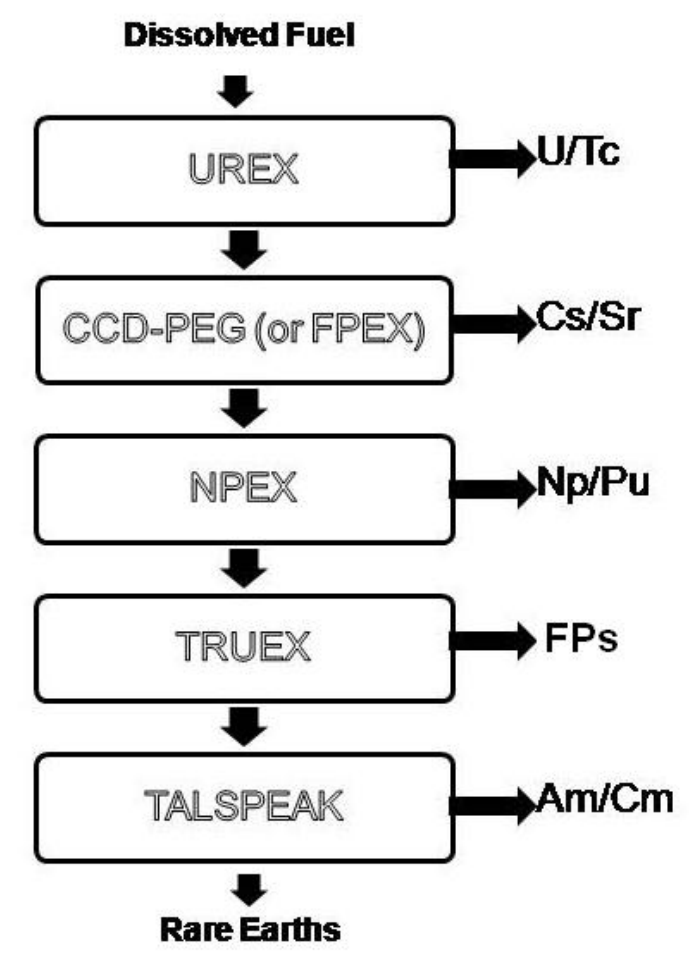

Figure 4-1. The UREX+ Process Used for this Study. [3] 
all AHA must be hydrolyzed prior to the FPEX step. This hydrolysis can be achieved by heating the stream containing AHA to increase the hydrolysis rate. Both the FPEX and CCD-PEG steps remove cesium and strontium but use different solvent systems to achieve the same result. FPEX is said to be more efficient in removing both cesium and strontium simultaneously, thereby requiring fewer stages and less equipment. By the NPEX step, all AHA must be hydrolyzed to allow plutonium to be extracted. NPEX uses the same solvent system (TBP-dodecane) as UREX. TRUEX follows NPEX and recovers anthanides and lanthanides rejecting the fission products using a CMPO-TBP-n-dodecane solvent. [2] The last step of the process is TALSPEAK, replacing Cyanex-301; it removes rare earth metals leaving the americium and curium and a nitric acid waste stream as product streams. In the most likely form of TALSPEAK, the organic stream from TRUEX will be stripped into aqueous, then treated with $0.8 \mathrm{M}$ di-(2-ethylhexy) phosphoric acid (HDEHP) dissolved in diisopropylbenzene (DIPB) that is capable of removing both actinides and lanthanides. This new organic stream will then be treated with diethylene triamine pentaacetic (DTPA) in aqueous solutions of either lactic or citric acid to remove actinides from the lanthanides. [4]

\subsection{Acetic Acid Removal Step}

Previous work by the authors was done to study the removal of acetic acid from a solution of acetic acid, nitric acid, and water-important components in the UREX+ process waste stream. [1] In an earlier study, solvent extraction was determined to be the best method to explore further. Tributyl phosphate-dodecane solvent was selected as a solvent system which provided both sufficient distribution coefficients and separation factors with an aqueous system 
of nitric acid at $0.5 \mathrm{M}$ and acetic acid at $0.05 \mathrm{M}$. [1] In addition, more tests were run at various TBP concentrations in dodecane as well as adjustments to the aqueous phase of $1 \mathrm{M}$ nitric acid with $0.1 \mathrm{M}$ acetic acid to prepare samples for analysis. A summary of all results for the selected solvent system of TBP-dodecane are shown in Table 4-1 and figure 4-2. This figure shows that distribution coefficients are directly related to the concentration of TBP in the solvent. The higher the concentration of the TBP, the higher the distribution coefficient and the higher the separation factor between acetic and nitric acids. Typically, TBP is a very viscous liquid nonideal for a centrifugal contactor used for solvent extraction steps in the UREX+ process.

However, dilute mixtures of TBP in dodecane can be used in the contactors since the presence of dodecane creates a much less viscous phase. A flow sheet of a proposed acetic acid removal step can be seen in figure 4-3.

\subsection{Extraction Factors}

Extraction factors are a way to compare distribution coefficients of two separate solutes to determine which is extracted more effectively. Since no testing has been done on UREX+ process demonstrations to determine the amount of acetic acid being extracted in each of the 
Table 4-1. Distribution Coefficients for the TBP-dodecane Solvent System[1]

\begin{tabular}{|c|c|c|c|c|}
\hline $\begin{array}{c}\text { AQUEOUS } \\
\text { SYSTEM }\end{array}$ & ORGANIC SYSTEM & $\begin{array}{c}\mathrm{K}_{\mathrm{D}} \text { Nitric } \\
\text { Acid }\end{array}$ & $\begin{array}{c}\mathrm{K}_{\mathrm{D}} \text { Acetic } \\
\text { Acid }\end{array}$ & $\begin{array}{c}\text { Separation } \\
\text { Factor }\end{array}$ \\
\hline & Dodecane & 0.022 & -0.021 & N/A \\
$0.5 \mathrm{M}$ Nitric Acid & 1M TBP in dodecane & 0.049 & 0.183 & 3.73 \\
0.05M Acetic & $1.5 \mathrm{M}$ TBP-dodecane & 0.098 & 0.878 & 8.96 \\
Acid Water & 2.5M TBP-dodecane & 0.308 & 1.481 & 4.81 \\
& TBP & 0.62 & 2.573 & 4.15 \\
\hline & Dodecane & -0.003 & -0.043 & N/A \\
1M Nitric Acid & 1M TBP-dodecane & 0.078 & 0.342 & 4.38 \\
$0.1 \mathrm{M}$ Acetic Acid & $1.5 \mathrm{M}$ TBP-dodecane & 0.15 & 0.525 & 3.50 \\
Water & $2.5 \mathrm{M}$ TBP-dodecane & 0.299 & 1.038 & 3.47 \\
& TBP & 0.66 & 1.751 & 2.65 \\
\hline
\end{tabular}

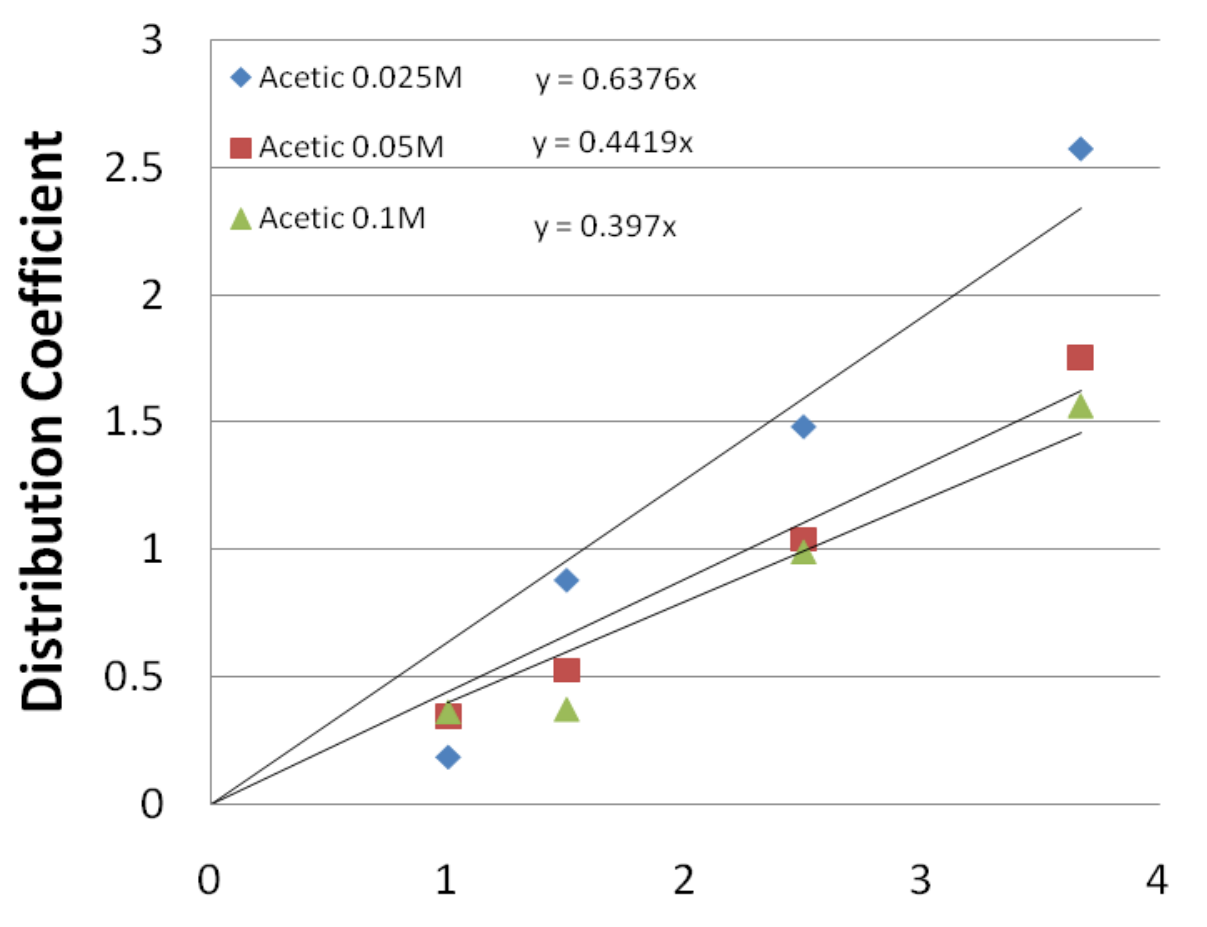

\section{TBP Concentration in Dodecane (Mol/L)}

Figure 4-2. Distribution Coefficients for Acetic Acid in Acetic Acid and Nitric Acid Mixtures vs. TBP Concentration. 


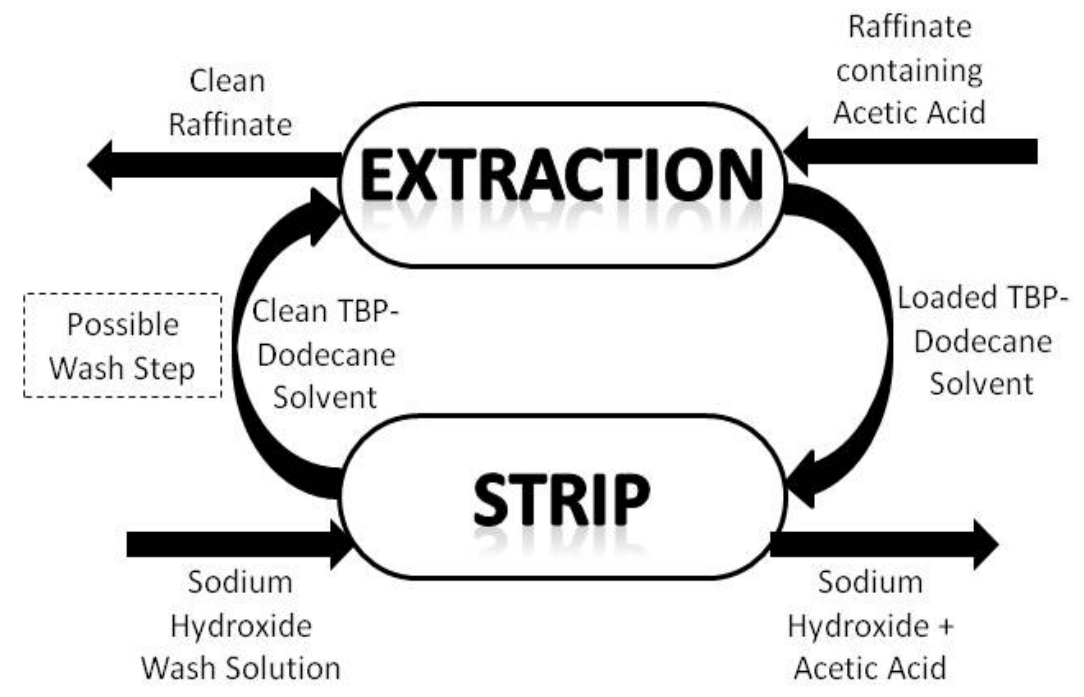

Figure 4-3. Acetic Acid Removal Flow sheet Step.

process steps, equation 1 will be used to compare the extraction of acetic acid in experimental tests including nitric acid and water to extraction of radioactive metals being extracted in each step of the UREX+ process.

$$
E=K_{D} \bullet \frac{O R G}{A Q}
$$

In this equation, $\mathrm{K}_{\mathrm{D}}$ is the distribution coefficient, relating the equilibrium concentration (mol/L) of the extracted component (solute) in the organic phase to the concentration $(\mathrm{mol} / \mathrm{L})$ of the solute in the aqueous phase. The $\mathrm{ORG} / \mathrm{AQ}$ is the organic to aqueous ratio of the volumetric flow rates. Therefore, the extraction factor (E) is a measure of moles of acid in the organic divided by moles of acid in the aqueous feed. The extraction factor multiplied by the concentration in the aqueous feed is simply the concentration of acetic acid in the organic phase. For example, in the NPEX step, plutonium and neptunium are the main components extracted. To evaluate the quantities of acetic acid extraction, a distribution coefficient will be needed, along with an organic to aqueous phase ratio. The product of the two values gives the extraction factor. If the 
organic to aqueous ratio to be used in $\mathrm{UREX}+$ is not given in literature, an extraction factor of 1 will be assumed to calculate the organic to aqueous ratio. The actual values used are expected to be only slightly more than this, but not a large multiple of this. Thus an estimate of the extraction can be obtained, even if it may be a little low. Using the same organic to aqueous ratio for extraction of the metals, an extraction factor can be found for acetic acid using the distribution coefficients found for acetic acid at the same concentration of TBP. The extraction factor for acetic acid is the fraction of acid extracted if the organic coming from the first stage is in equilibrium with the acetic acid in the feed. Therefore, for these calculations, it is assumed a large number of stages are used (i.e. infinite) thus giving generous values as maximum amounts that acetic acid will interfere with each step of the UREX+ process. Using the distribution coefficients found from figure 4-2 and the organic to aqueous ratios from the metal extraction calculations, these extraction factors will determine how much acetic acid is expected to be extracted during each metal extraction step and, thus, an estimate of whether acetic acid will extract sufficiently to cause interference in the extraction of these metals.

\section{Acetic Acid Interference Estimates}

Each step in the UREX+ process is examined closely to determine if any problems are expected to arise with acetic acid being present in that step. Since no data have been obtained for the acetic acid content in actual test runs of the UREX+ process, the authors rely on related work to estimate the acetic acid extraction and see if that amount of acetic acid is likely to consume (or bind to) a significant fraction of the extractant. 


\subsection{UREX}

The two main factors in this step of the process to be examined are AHA concentration and uranium extraction. Acetohydroxamic acid is added in two places within the UREX step. First, it is added in the feed to suppress extraction of plutonium. Additional AHA is then added to a scrub step, so AHA from both additions will be present for the feed in the CCD-PEG step. AHA is not expected to hydrolyze significantly in this step since most will be bound to plutonium, however small amounts of hydrolyzation could occur. Acetohydroxamic acid forms acetic acid and hydroxylamine nitrate, and has the rate equation shown in equation 2.

$$
-\frac{d[A H A]}{d t}=k[A H A]\left[H^{+}\right]
$$

This equation has been tested at $25^{\circ} \mathrm{C}$ with various nitric acid concentrations ( 0 to $\left.4 \mathrm{M}\right)$ and shows increased hydrolysis as the nitric acid concentration increases. The rate equation has a $\mathrm{k}$ $=0.0015 \mathrm{~mol} / \mathrm{L} * \min$ and an activation energy of $81.4 \mathrm{~kJ} / \mathrm{mol}$. [5]

The uranium extraction values versus the concentration of the feed are shown in figure 44. These values are for a system of $0.726 \mathrm{M}$ TBP-kerosene and $0.5 \mathrm{M}$ nitric acid with $0.126 \mathrm{M}$ uranium initially. Kerosene is very similar to dodecane and is assumed to behave similarly. If we use equation 1 and assume an extraction factor of 1.0 and a $K_{D}$ of 1.33 , then the organic to aqueous feed ratio will be from 0.752 . Using this same equation, the data for acetic acid distribution in $0.5 \mathrm{M}$ nitric acid, and the organic to aqueous feed ratios, the extraction factor for acetic acid from figure $4-2$ is 0.241 . That is, only approximately $24 \%$ of the small concentration of acetic acid in the UREX process stream will be removed. Since the acetic acid concentration is expected to be much smaller than the uranium concentration, the acetic acid is not likely to "tie- up" a significant fraction of the TBP and hinders the extraction of uranium. If the uranium 
is purified further by other steps, the role of acetic acid may be eliminated completely. Note also that much of the small quantity of acetic acid extracted during UREX will be washed out in the scrub step; so the impact of the traces of acetic acid should be even less than indicated above.

\subsection{CCD-PEG (or FPEX)}

Two options are available for the removal of cesium and strontium-CCD-PEG and FPEX. The CCD-PEG step uses a $0.08 \mathrm{M}$ chlorinated cobalt dicarbollide (CCD) to remove cesium, and $0.016 \mathrm{M}$ polyethylene glycol (PEG-400) to remove strontium. These extractants are in a phenyltrifluoromethyl sulfone (FS-13) diluent. Distribution coefficients for both Cs and $\mathrm{Sr}$ are extremely high and favorable for an aqueous system of nitric acid concentrations ranging

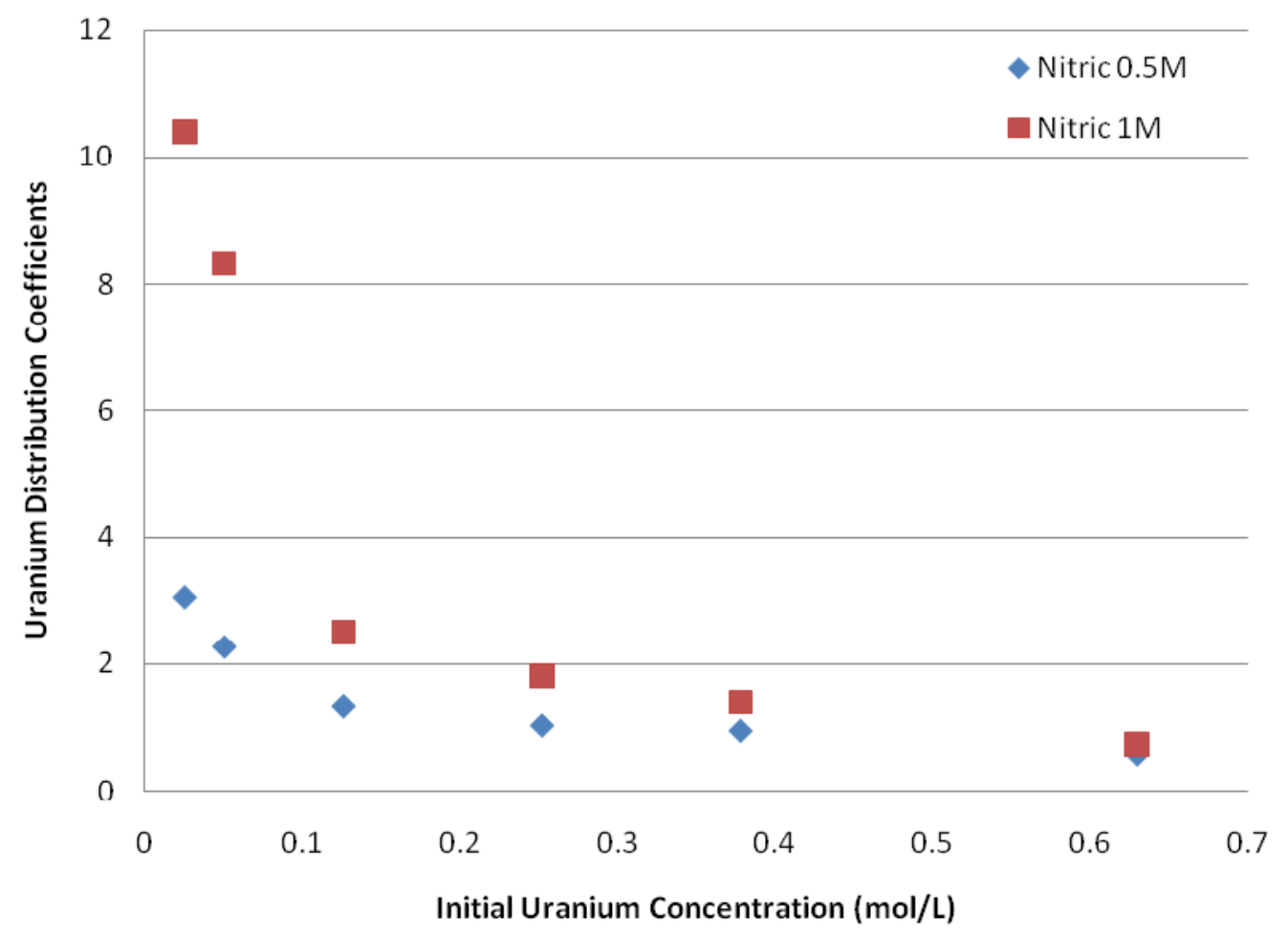

Figure 4-4. Uranium Distribution Coefficients vs. Initial Uranium Concentrations at different Nitric Acid Feed Concentrations. [6] 
from $0.1 \mathrm{M}$ up to about $10 \mathrm{M}$. However, the distribution coefficients are inversely proportional to the nitric acid concentration. As nitric acid concentration increases, the distribution coefficient decreases over the range of two orders of magnitude. The effect of AHA on this system is explored in the paper by Law, et. al. [7] Since AHA is added to the feed and scrub step in the UREX for the feed to the subsequent CCD-PEG step, this analysis is necessary to identify any problems in the extraction of cesium or strontium. Both fresh AHA and decomposed AHA-a mixture of AHA and hydrolysis components of acetic acid and hydroxyl amine nitrate - were tested. In the case of fresh AHA, there was little to no difference in cesium or strontium distribution coefficients. However, in the case of decomposed AHA, the cesium distribution coefficients were unchanged while the strontium distribution coefficients decreased significantly. Decomposed AHA could be AHA, acetic acid, or hydroxyl amine nitrate interfering with the strontium extraction. During this study, it could not be determined which of the three was interfering. [7] Therefore, acetic acid can possibly cause interference at this step if acetic acid is responsible for the strontium extraction decrease. Thus, if little decomposition occurs before the CCD-PEG step, there will be little effect on strontium extraction. On the other hand and more likely, if HAN (and not acetic acid) is responsible for the decrease in strontium extraction, it may be worthwhile to decompose the AHA all the way to acetic acid prior to the CCD-PEG step. Determining the component responsible for reducing strontium extraction was beyond the scope of this study. It will be assumed acetic acid does not interfere with this step in further analysis. Another possibility for removal of cesium and strontium is the FPEX step. This step involves a cooperative extraction of strontium and cesium using 4,4',(5')-di-(t-butyldicyclohexano)-18-crown-6 (DtBuCH18C6), calix[4]arene-bis-(tert-octylbenzo-crown-6) 
(BOBCalixC6), 1-(2,2,3,3-tetrafluoropropoxy)-3-(4-sec-butylphenoxy)-2-propanol (Cs-7SB modifier), and Isopar ${ }^{\circledR}$ L solvent. The DtBuCH18C6 extracts strontium while the BOBCalixC6 extracts cesium. The Cs-7SB modifier is the component which makes it possible to extract strontium and cesium in one step rather than two (SREX and CSSX) steps. The BOBCalixC6 has a very high affinity for cesium and, since acetic acid is a much smaller molecule, it is not expected to bind in cesium's place. There was nothing found in literature to suggest acetic acid present in the FPEX process will cause any problems with the extraction of cesium or strontium. Therefore, it will be assumed there is no problem with acetic acid's presence in FPEX. [3]

\subsection{NPEX}

The two components being extracted in this step are neptunium and plutonium. Both neptunium and plutonium distribution coefficients are higher than those for acetic acid in a TBPdodecane solution. In $30 \mathrm{vol} \%$ TBP-dodecane with $1 \mathrm{M}$ nitric acid and a 1:1 organic to aqueous ratio, the $\mathrm{K}_{\mathrm{D}}$ for $\mathrm{Np}(\mathrm{VI})$ is given as 6.4 while $\mathrm{Np}(\mathrm{IV})$ is given as 1.0. At an organic to aqueous ratio of 1 , the extraction factors for these two components are 6.4 and 1.0 respectively. [8] Since it is assumed that nitric and acetic acid has a 10:1 molar ratio, [9] the distribution coefficient for acetic acid at $0.1 \mathrm{M}$ in the presence of $1 \mathrm{M}$ nitric acid is 0.437 based on the correlation in figure 42. Using the organic to aqueous ratio of 1 , the extraction factor of acetic acid is 0.437 . Based on the previous discussion of extraction factors, this extraction factor means about $44 \%$ of acetic acid is removed from the aqueous phase into the organic stream. Since acetic acid binds with TBP in a 1:1 ratio [10], it will only use $4 \%$ of the TBP in the stream. This is not expected to affect the neptunium or plutonium extraction more than slightly.

In several papers [11] [12] [13], the concentration of Pu used in various fuel cycle experiments ranges from $0.02 \mathrm{~g} / \mathrm{L}$ to $34 \mathrm{~g} / \mathrm{L}$. The distribution coefficient most recently obtained 
is from a 2007 flow sheet test. The NPEX step was simulated with a distribution coefficient of 6.5 for Np and approximately 11 for Pu. [14] Since distribution coefficients depend highly on the nitric acid concentration, it can be assumed that the nitric acid concentration is near the same as for the above experiments ran with Neptunium at $1 \mathrm{M}$ nitric acid. With this assumption, acetic acid distribution coefficients can also be compared to the Pu distribution coefficient. Using the same $\mathrm{O} / \mathrm{A}$ ratio as used for neptunium, 1 , the $\mathrm{Pu}$ extraction factor is 11 . Using this same $\mathrm{O} / \mathrm{A}$ ratio for acetic acid, the extraction factor for acetic acid is 0.4367 . This means $44 \%$ of acetic acid present at this point of the process will be extracted. The amount of TBP used by acetic acid is estimated to be $4 \%$ of TBP. Even though this small amount of TBP could be used by acetic acid, acetic acid is not expected to cause a substantiating effect on plutonium extraction.

\subsection{TRUEX}

The purpose of this step is to remove essentially all transuranic elements from the spent nuclear fuel. The main elements removed in this step are americium and curium. However, for these studies, only americium will be considered since curium is more easily extracted. These two components are removed by the TRUEX solvent made up of $0.2 \mathrm{M}$ CMPO-1.4M TBPdodecane solution. We know that the TBP extracts acetic acid, but the effects of CMPO on the extraction were not known; so acetic acid distribution coefficient measurements were made with the TRUEX solvent. The model aqueous solution used consisted of $0.5 \mathrm{M}$ nitric acid and $0.05 \mathrm{M}$ acetic acid. Higher concentrations were not tested due to a possible third phase formation. The results of these tests are shown in Table 4-2. In the literature, americium extraction is tested in a system of $0.2 \mathrm{M}$ CMPO-1.4M TBP-Conoco (C12-C14). Data at $30^{\circ} \mathrm{C}$ and about $0.5 \mathrm{M}$ nitric acid give a distribution coefficient of 8.9 for americium. [15] Assuming an extraction factor of 1 and 


\section{Table 4-2. Acid Distribution in TRUEX Solvent.}

\begin{tabular}{|c|c|}
\hline Acid & $\mathbf{K}_{\mathbf{D}}$ \\
\hline Acetic Acid $0.05 \mathrm{M}$ & 0.952 \\
Nitric Acid $0.5 \mathrm{M}$ & 0.328 \\
\hline
\end{tabular}

using equation 2 for calculation of the flow ratios, the organic to aqueous ratio can be determined. The $\mathrm{O} / \mathrm{A}$ ratio is found to be 0.112 . Utilizing this ratio and the distribution coefficient correlated for acetic acid using a comparable diluent dodecane from figure 4-2, the extraction factor for acetic acid is 0.107 . This corresponds to $10.7 \%$ of acetic acid extracted and only a maximum of $0.38 \%$ of TBP consumed in acetic acid extraction reaction. With this small value, the extraction of americium should not be affected by acetic acid to any substantial amount.

\subsection{TALSPEAK}

TALSPEAK separates the lanthanides from the actinides that are extracted by the TRUEX process. The process is done in a two steps. The organic solvent system is made up of $0.8 \mathrm{M}$ di(2-ethylhexy) phosphoric acid (HDEHP) dissolved in diisopropylbenzene (DIPB). This extracts both lanthanides and actinides. The aqueous system of diethylene triamine pentaacetic acid mixed with either lactic or citric acid is added to strip the actinides from the organic, thereby sufficiently separating the actinides from the lanthanides. [4] The organic stream from the TRUEX step is treated to strip actinides and lanthanides into the aqueous phase. Then lanthanides and actinides are both extracted into the organic stream by the HDEHP solvent system. DTPA then extracts the actinides from the organic phase. This process can also be accomplished by treating the aqueous stream (treated TRUEX organic stream) with the DTPA 
solution to hold the actinides in the aqueous stream and then extracting lanthanides by HDEHPDIPB. To determine if acetic acid would interfere if it were to be at all present, acetic acid extraction tests were done for the system of 0.8M HDEHP in DIPB and for 0.3M HDEHP-0.2M TBP-dodecane. For both tests, no extraction of acetic acid or nitric acid occurred. Therefore, there is no expected interference with acetic acid being in the streams during the TALSPEAK process if any were present in this step.

\section{Analysis/Discussion}

A flow sheet is proposed from the results of the literature review. (figure 4-5) The literature review and experimental data on acetic acid extraction in the process solvents suggests no likely interference of acetic acid in the process. The flow sheet reflects this finding with the acetic acid removal placed at the end of the process when nitric acid is concentrated for the recycle.

While ideally one would like the option to place the acetic acid removal step anywhere in the process with no interference on its part, there are complications with using TBP-dodecane solvent for extracting acetic acid at some positions in the UREX+ process because the solvent would extract metals (radioactivity) as well as acetic acid if the acetic acid removal step were placed prior to the step in the UREX+ process where the metals are to be removed. For example, placing the TBP-dodecane base acetic acid removal step just after the UREX step would result in extraction of plutonium and neptunium as well as acetic acid. As shown in the literature review, these metals are favored for extraction by TBP over acetic acid so they will be the first to be 
removed. Therefore, placing this step before neptunium and plutonium extraction (NPEX) would not be an efficient option. Placing the removal step after NPEX may be suitable because

Dissolved Fuel

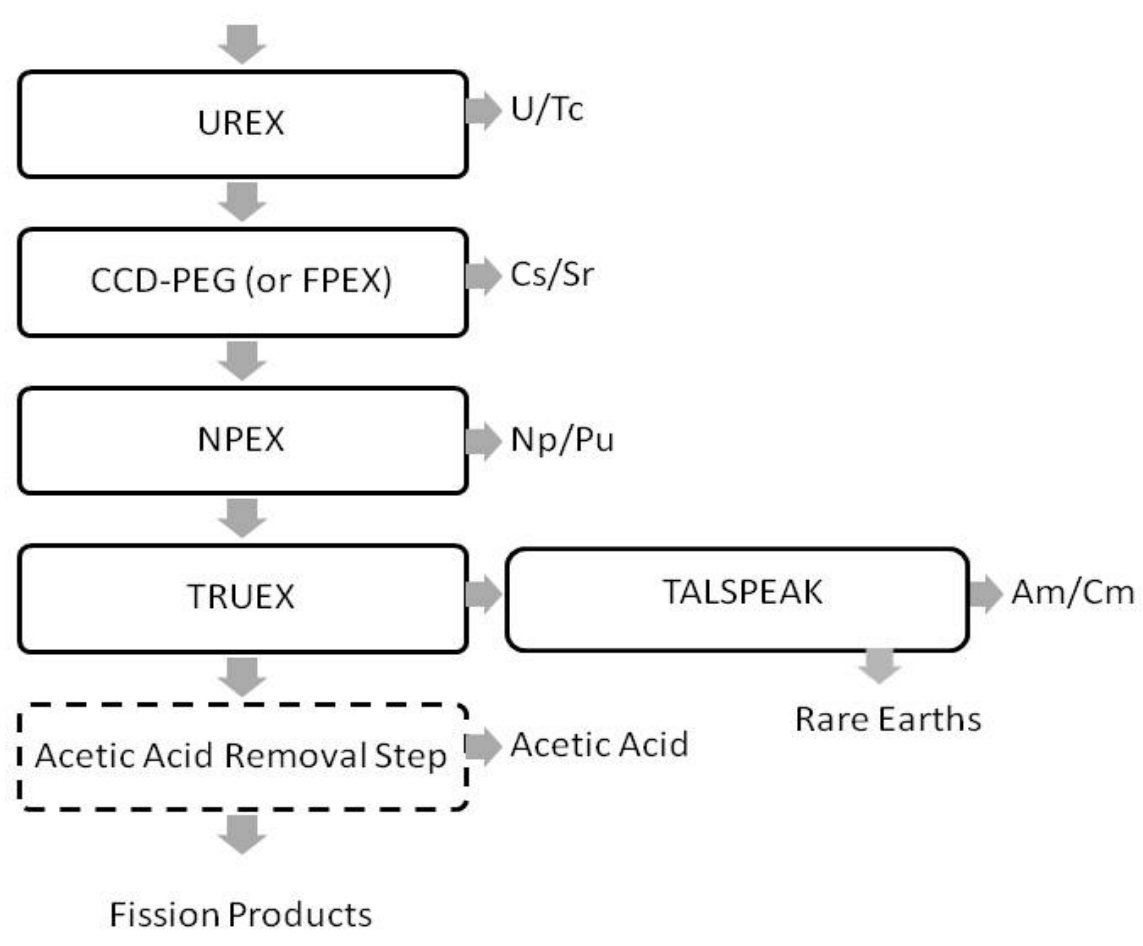

Figure 4-5. Flowsheet Proposal.

americium and curium are mainly extracted by CMPO and not the TBP, other fission products will be extracted with the acetic acid. Placing the removal step between TRUEX and TALSPEAK would be a possible method since the aqueous stream is fission products plus acetic acid and nitric acid. The fission products were not extracted by CMPO-TBP-dodecane system, therefore, they will most likely not be affected by the TBP-dodecane solvent used to treat acetic acid. Therefore, the simplest model is the flow sheet proposed in figure 5 which shows the acetic 
acid removal step after all metal extraction steps have taken place to eliminate concern for extraction of metals in the same process.

\section{Conclusion}

After literature review of each process step and analysis of acetic acid extraction compared with radioactive metal extraction, it is determined that acetic acid should not pose a problem for any step of the UREX + process. Thus, acetic acid is principally a problem for recycle of the nitric acid. Hypothetically, the acetic acid solvent extraction removal step can be placed anywhere in the process. However, there is cause for concern that metals will extract with the removal step if placed before these metals are removed. Therefore, due to simplicity, the authors feel the removal step should go after TRUEX where distillation may be a viable removal option in the combination of re-concentrating nitric acid for recycle.

\section{Acknowledgements}

This work was supported by the U.S. Department of Energy, NERI program, under DOE Contract No. DE-PS07-05ID14713. 


\section{REFERENCES}

1. George F. Vandegrift, Monica C. Regalbuto, Scott B. Aase, Hassan A. Arafat, Allen J. Bakel, Delbert L. Bowers, James P. Byrnes, Mark A. Clark, Jeffrey W. Emory, John R. Falkenberg, Artem V. Felix, Lohman D. Hafenrichter, Ralph A. Leonard, Candido Pereira, Kevin J. Quigley, Yifen Tsai, Mark H. Vander Pol, and James J. Laidler. $227^{\text {th }}$ ACS National Meeting. Anaheim, CA. 2004.

2. C. L. Riddle, J. D. Baker, J. D. Law, C. A. McGrath, D. H. Meikrantz,.Sol. Extr. \& Ion Exch.23, 449 (2005).

3. G. D. Del Cul, L.M. Toth, W.D. Bond, G.D. Davis, S. Dai.Sep. Sci. Tech., $\underline{1-4}, 431$. (1997)

4. Jessica Mitchell, Jared Johnson, Robert M. Counce, Jack S. Watson, Barry B. Spencer, G.D. Del Cul. Sep.Sci.\&Tech. $\underline{43}$, 2537. (2008)

5. Dong Yong Chung and Eli Hee Lee. J. Ind. Eng. \& Chem., 12, 962.(2005)

6. Jamal Stas, Ajaj Dahdouh, and Habib Shlewit. Per. Poly. Ser. Chem. Eng.,ㅆ99, 3. (2005)

7. Jack D. Law, R. Scott Herbst, Dean R. Peterman, Rich D. Tillotson, and Terry A. Todd, Nucl.Tech.,147, 284. (2004)

6. Dong Yong Chung and Eli Hee Lee. J. Alloys \& Cpds, $\underline{451}$, 440. (2008)

7. L. Nunez and G.F. Vandegrift. ANL-00/35. (2001)

8. A.S. Kertes and P.J. Lloyd. Journal of the Chemical Society, May,3477. (1965)

9. B. Goldschmidt, P. Regnaut, and I. Prevot. Commissariat a l'Energie Atomique. s.1. : Rapport CEA-R - France, p.492. (1955)

10. G.F. Best, H.A.C. McKay, and P.R. Woodgate. J. Inorg. Nuc. Chem., Vol. 4, pp. 315. (1957)

11. V.E. Vereshchagin, and E.V. Renard., Atomnaya Energiya, 45, 45. (1978) 
12. Artem V. Gelis, Delbert M. Bowers, Andrew S. Hebden, Candido Pereira, Monica Regalbuto, and George F. Vandegrift. Park City, UT : s.n., 32nd Actinides Separation Conference. (2008)

13. E.P. Horwitz, D.C. Kalina, H. Diamond, G.F. Vandegrift, and W.W. Shultz. Sol. Ext. \& Ion Exch. $\underline{3}, 75 .(1985)$ 


\title{
Chapter V: Water Solubility of Organic Solvents and Their Relationship to the Extraction of Nitric and Acetic Acid from UREX+ Streams
}

\author{
Russell T.H., Counce R.M., and Watson J.S. \\ University Of Tennessee Knoxville, Knoxville, TN, US \\ Spencer B.B. and Del Cul G.D. \\ Oak Ridge National Laboratory, Oak Ridge, TN, US
}




\section{Introduction}

This study investigates the solubility of water in various solvents and solvent-mixtures being considered for the counter-current solvent extraction of acetic acid from UREX+ process solutions and seeks to determine if there is any correlation between equilibrium water solubility and equilibrium solvent extraction behavior.

In the UREX+ process for treating spent nuclear fuel, acetic acid appears in some of the process streams as a degradation product of acetohydroxamic acid (AHA). AHA is used in the UREX+ process to suppress the co-extraction of plutonium and uranium in the UREX step. To recycle the nitric acid solution and to reduce problems in downstream process steps, the removal of acetic from process streams is being studied.

The solubility of water in the organic phase (solvent) is the concentration of water in the organic phase after equilibrium has been established between the aqueous phase (aq) and organic phase (o) and can be defined by the following equations where $\mathrm{C}_{\mathrm{w}}$ is the concentration of water, $a_{w}$ is the activity of water, and $\gamma_{w}$ is the activity coefficient of water.

$$
\begin{aligned}
& \mathrm{a}_{\mathrm{w}, \mathrm{o}}=\gamma_{\mathrm{w}, \mathrm{o}} \mathrm{C}_{\mathrm{w}, \mathrm{o}} \\
& \mathrm{a}_{\mathrm{w}, \mathrm{aq}}=\gamma_{\mathrm{w}, \mathrm{aq}} \mathrm{C}_{\mathrm{w}, \mathrm{ag}}
\end{aligned}
$$

At equilibrium, the activities of water in the organic and aqueous phases are equal, and therefore,

$$
\begin{aligned}
& \mathrm{a}_{\mathrm{w}, \mathrm{o}}=\mathrm{a}_{\mathrm{w}, \mathrm{aq}} \\
& \gamma_{\mathrm{w}, \mathrm{o}} \mathrm{C}_{\mathrm{w}, \mathrm{o}}=\gamma_{\mathrm{w}, \mathrm{aq}} \mathrm{C}_{\mathrm{w}, \mathrm{aq}}
\end{aligned}
$$




$$
\begin{gathered}
\mathrm{C}_{\mathrm{w}, \mathrm{o}}=\mathrm{C}_{\mathrm{w}, \mathrm{aq}}\left(\gamma_{\mathrm{w}, \mathrm{aq}} / \gamma_{\mathrm{w}, \mathrm{o}}\right) \\
\text { Or } \\
\mathrm{C}_{\mathrm{w}, \mathrm{o}}=\left(\mathrm{a}_{\mathrm{w}, \mathrm{aq}} / \gamma_{\mathrm{w}, \mathrm{o}}\right)
\end{gathered}
$$

The water content in this paper is defined as moles of water per liter of dry organic solution. An estimate of mole fractions can be made by assuming the low water solubility in the organic phase do not greatly affect the density of the pure organic solutions.

Throughout the study of acetic acid removal from UREX+ streams, a variety of different solvents were considered, however, priority was given to organic solvents already utilized in the UREX + process, thereby simplifying process design. The solvents studied included n-Dodecane (nDD), 1, 2 Dichloroethane (DCE), and Phenyltrifluoromethyl Sulfone (FS-13) and mixtures of these solvents with Tributyl Phosphate (TBP). DCE is used as a comparative solvent and is not considered for possible use in the UREX+ process because of its vapor pressure, flammability, and toxicity. The other solvents are likely to be used along with TBP in future nuclear fuel reprocessing technologies as well as the UREX+ process $^{(1)}$. The water solubility information in the presence of acetic acid is of particular interest if it could be related to solvent performance in extracting acetic acid. The objective of this work is to determine water content and analyze any possible relationships between water content and acetic acid extraction by comparing equilibrium water content with equilibrium acetic acid content.

\section{Literature Review}

The continued use of the PUREX process for treating spent nuclear fuel has led to numerous studies focused on the competitive equilibrium between TBP, nitric acid, and water, 
and various models have been presented to account for the complexity of the extraction interactions with varying TBP concentrations. These studies include multiple conclusions about the nature of the $\mathrm{TBP} / \mathrm{diluent} / \mathrm{H}_{2} \mathrm{O}$ and $\mathrm{TBP} /$ diluent $/ \mathrm{HNO}_{3} / \mathrm{H}_{2} \mathrm{O}$ complexes that may form with some work postulating that the dimmer, $\mathrm{TBP}_{2}$, and possibly the trimmer, $\mathrm{TBP}_{3}$, play a role in the equilibrium with water and nitric acid ${ }^{(2,3,4,5)}$.

Previous work has also focused on the behavior of water in TBP-diluent mixtures, including kerosene and $\mathrm{n}$-dodecane ${ }^{(6,7,8)}$. Friehmelt et. al. examined the addition of $\mathrm{n}$-dodecane into the organic phase and determined through HNMR spectroscopy that water is expelled from the organic phase with the addition of a hydrocarbon diluent. The work of Roddy with n-octane TBP mixtures confirms a drop in water content with the addition of an organic diluent. Friehmelt et. al. also concluded that while the overall water extraction decreased, the strength of the bonds between water and the organic phase increase with an increase in organic diluent concentration.

As far as the effects of acid co-extraction on water content, the work of Pushlenkov et. al. with equilibrium water content in the presence of nitric acid concluded that the presence of nitric acid in the aqueous phase does not reduce the equilibrium water content ${ }^{(9)}$.

Previous work on acetic acid extraction from nitric acid solutions in UREX+ by Mitchell et. al. focused on a dilute mixture of nitric acid and acetic acid in a 10:1 ratio consisting of approximately $0.25 \mathrm{M}$ nitric acid and $0.025 \mathrm{M}$ acetic acid. They concluded that a $1.5 \mathrm{M}$ TBP in nDodecane solution provided the best extraction results when comparing mixtures of TBP/DCE and TBP/FS-13.

The work presented here was done in order to further examine the effects of water and mixtures of TBP and three different organic diluents (n-Dodecane, 1,2 Dichloroethane, and FS- 
13) on the water content of the organic phase. Multiple TBP concentrations where used with more emphasis placed on TBP/n-Dodecane because of its success in practical acid extraction application. Also, studies focused on the water content of the organic phase after contact with pure water and an aqueous phase consisting of an approximately $0.25 \mathrm{M}$ nitric acid and $0.025 \mathrm{M}$ acetic acid, in accordance with the extraction research, to provide experimental water content data for the duel acid mixture.

The Karl Fisher method of water content analysis was employed for data acquisition after literature research concluded that it was the most popular and accepted method used in earlier work $^{(10)}$.

\section{Experimental Equipment and Procedures}

After reviewing the equipment options for Karl Fischer titration, the Hydranol Moisture TestKit ${ }^{\circledR}$ produced by Sigma-Aldrich was chosen. The kit consists of two glass titration vessels, each with an airtight septum, syringes with needles, Hydronal Solvent-E, used to create a consistent testing environment by mixing with the substance being tested, Hyronal Titrant which contains iodine and sulfur dioxide, and Hydranol Standard 5.00 for standardizing the Titrant. It utilizes the oxidation of sulfur dioxide by iodine with consumption of water and uses ethanol based reagents. The Hydronal Solvent-E is placed in the titration vessel and is titrated to dryness using Hydranol Titrant Compounent of known concentration. The end point is indicated by a sharp change from approximately clear to opaque. The sample is then introduced into the titration vessel through the septum by means of a syringe, and the mixture is titrated again to determine the water content. All samples of new materials (those with no known literature value 
to compare) were titrated in triplicate or greater, and the concentration of Hydranol Titrant Component was tested before each different sample. The equations for determining the water content $\left(\mathrm{C}_{\mathrm{w}, \mathrm{o}}\right)$ from Titrant usage and for determining the Titrant concentration $\left(\mathrm{T}_{[\mathrm{C}]}\right)$ are given below.

$\mathrm{C}_{\mathrm{w}, \mathrm{o}}=\left[\right.$ Consumption of Titrant $[\mathrm{ml}] *$ Titre $\left.\left[\mathrm{mg} \mathrm{H}_{2} 0 / \mathrm{ml}\right]\right] /[$ sample volume $[\mathrm{ml}]] /\left[18\left[\mathrm{~g} / \mathrm{mol} \mathrm{H}_{2} 0\right](7)\right.$

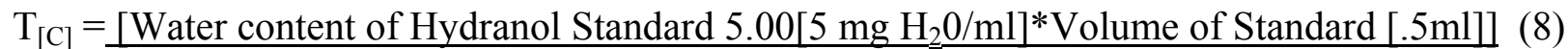
[Consumption of Titre [ml]]

The 99\% dodecane and TBP were obtained from ACROS; 99\% 1,2 dichloroethane was obtained from Fischer Scientific, and FS-13 was obtained from Marshallton Labs. All samples were used as received and were contacted with the aqueous phase in the same fashion. The aqueous/organic mixtures were placed on a stir plate set for vigorous mixing for two hours. They then received light mixing for an additional 4-6 hours, and finally, were taken off the stir plate and left overnight to allow separation of the aqueous and organic phases. All mixing and measurements were done at room temperature. Room temperature ranged from 24-26 degrees Celsius.

The approximately $0.25 \mathrm{M}$ nitric acid and $0.025 \mathrm{M}$ acetic acid combination was created by mixing equal volumes of $0.5 \mathrm{M}$ nitric acid and $0.05 \mathrm{M}$ acetic acid.

All plots and regressions were done in Microsoft Office Excel. 


\section{Results and Discussion}

\subsection{Water Solubility $\left(C_{w, 0}\right)$ Results from Contact Between De-ionized Water and TBP- Organic Diluent Mixtures}

Molarities (M) of TBP are given in mol/L of "dry" organic, organic before equilibrium contact with water. These units will be used throughout the results for consistency and comparison purposes. A complete listing of results is given in Appendix A.

The solubility of water in all TBP/diluent mixtures is given in Figure 5-1 where the $\mathrm{x}$ axis is the molarity of the "dry" TBP solution and the y-axis is the average water solubility of the TBP/diluent mixture in mol/L. As seen from Figure 5-1, the water solubility increases dramatically with increasing TBP concentration, reaching a maximum at the composition of pure TBP, 3.67M TBP. It is worth noting that the pure diluent (0 M TBP) has a small water solubility.

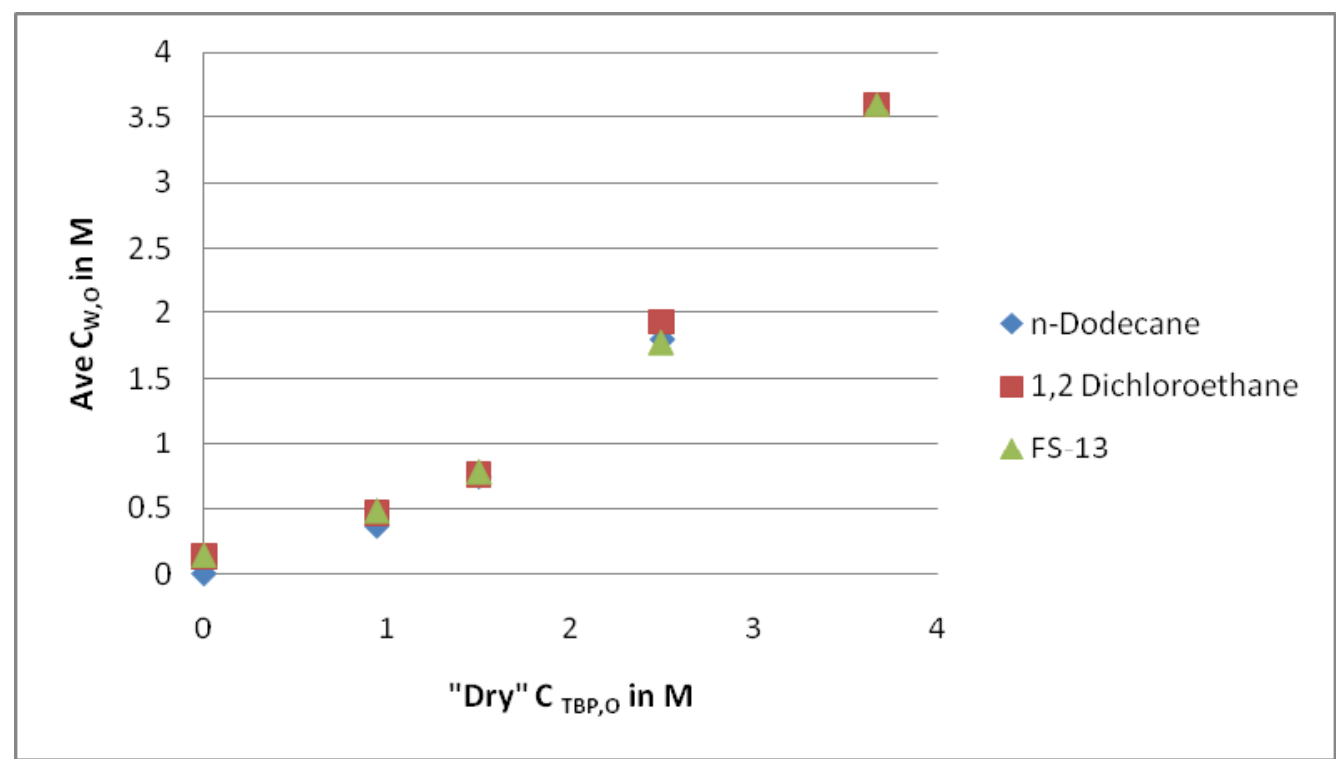

Figure 5-1. Effects of "Dry" $C_{\mathrm{TBP}, \mathrm{o}}$ on $\mathrm{C}_{\mathrm{W}, \mathrm{o}}$ for TBP/diluent mixtures 
The water solubility of the all of the TBP/diluent solutions seems to be in agreement with each other. The plots for n-Dodecane, 1,2 Dichloroethane, and FS-13 in Figure 5-1 seem to differ only slightly and display the same trend toward increased water content with increased TBP concentration.

All three diluents exhibit similar results in the water solubility tests. This suggests that the presence of TBP is the primary factor in water extraction. To gain further perspective into the types of interactions taking place between TBP and water in the equilibrium mixture, the log of both the $\mathrm{M}$ of TBP and $\mathrm{C}_{\mathrm{w}, \mathrm{o}}$ of the TBP/diluent mixtures were plotted in Figure 5-2. Obviously the zero TBP values were eliminated from the data. Therefore, only four data points were plotted for each TBP/diluent mixture. The slopes of the log-log plots for all three mixtures display similar numbers ranging from 1.6876 for $\mathrm{n}$-Dodecane to 1.5488 for 1, 2 Dichloroethane and 1.7427 for FS-13, and in fact, the data are so similar that the second data points for n-Dodecane and 1, 2 Dichloroethane are laying on top of each other. 


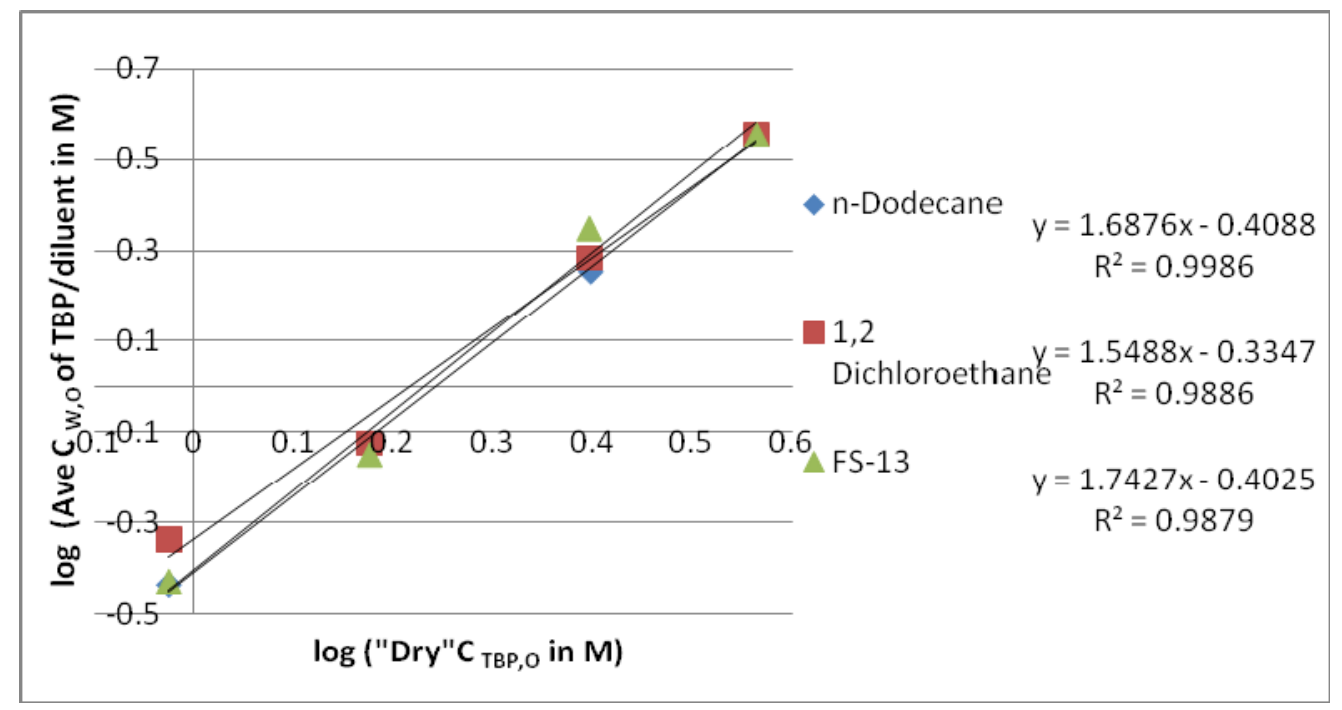

Figure 5-2. Log-log plot of average $C_{w, o}$ of TBP/diluent solutions

The range of slopes between 1.5 and 2 for all three TBP/diluent mixtures supports the conclusion that there exists a combination of TBP/water interactions ranging from $[\mathrm{TBP}]_{2}\left[\mathrm{H}_{2} 0\right]_{3}$ to $[\mathrm{TBP}]_{1}\left[\mathrm{H}_{2} \mathrm{O}\right]_{2}$ hydration complexes which would provide a slope between 1.5 and 2 for the log $-\log$ plots based on the equation

$$
\gamma_{\mathrm{w}, \mathrm{o}} \mathrm{C}_{\mathrm{w}, \mathrm{o}}=\mathrm{a}_{\mathrm{w}, \mathrm{a}} \gamma_{\mathrm{o}} \mathrm{k}\left(\mathrm{C}_{\mathrm{TBP}, \mathrm{o}}\right)^{\mathrm{N}} \text {. (9) }
$$

With the activity coefficients assumed to be one and the activity of water close to one at the acid concentrations in use, equation 9 simplifies down to

$$
\begin{gathered}
\mathrm{C}_{\mathrm{w}, \mathrm{o}}=\mathrm{k}\left(\mathrm{C}_{\mathrm{TBP}, \mathrm{o}}\right)^{\mathrm{N}}(10) \\
\text { and } \\
\log \mathrm{C}_{\mathrm{w}, \mathrm{o}}=\log (\mathrm{k})+\mathrm{N} \log \mathrm{C}_{\mathrm{TBP}, \mathrm{o}} \\
\text { where } \mathrm{N}=\text { the slope of the } \log -\log \operatorname{plot}^{(11)}
\end{gathered}
$$




\subsection{Water Solubility $\left(\mathrm{C}_{\mathrm{w}, 0}\right)$ Results after Contact with De-ionized Water containing approximately 0.25M Nitric Acid and 0.025M Acetic Acid with Various Concentrations of TBP in Organic Diluents}

Molarities (M) of TBP are given in mol/L of "dry" organic, organic before equilibrium contact with water. These units will be used throughout the results for consistency and comparison purposes. A complete listing of results is given in Appendix B.

After testing the effects of diluent selection on water solubility, experiments were performed to examine what affect the addition of approximately $0.25 \mathrm{M}$ nitric acid and $0.025 \mathrm{M}$ acetic acid might have on the water content of the organic phase at equilibrium. FS-13 was left out of this series of tests because of its expense and similar behavior to the two other diluents in the previous tests.

Much like the pure water aqueous tests, Figure 5-3 illustrates that the water content appears to be independent of the diluent selected. Comparing the values of $\mathrm{C}_{\mathrm{w}, \mathrm{o}}$ in the presence of the dual acid system to those in the pure water tests, the presence of nitric and acetic acid (at these concentrations) seems to have a very limited effect on the water content, and again both n- 
Dodecane and 1,2 Dichloroethane display similar results across the TBP concentrations tested.

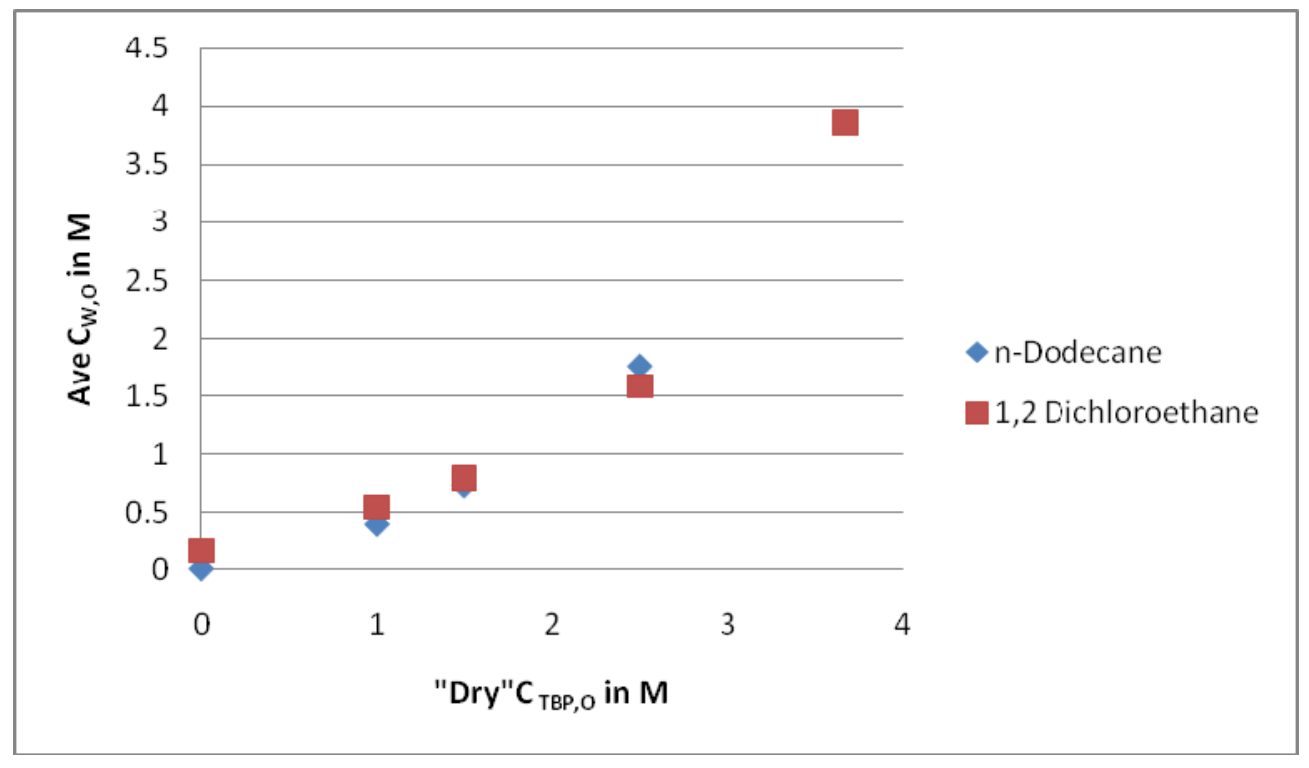

Figure 5-3. Average $\mathrm{C}_{\mathrm{W}, \mathrm{O}}$ of TBP/diluent solutions in the pressence of duel acid system

To confirm that the water content remained unaffected by the addition of the approximately $0.025 \mathrm{M}$ acetic acid and $0.25 \mathrm{M}$ nitric acid, the log-log plot of the water content versus TBP concentration was again created. As is seen in Figure 5-4, the slopes of the linear regressions are very similar to those in Figure 5-2. N-Dodecane has a slope of 1.7298 in Figure 5-4 and a slope of 1.6876 in Figure 5-2, and 1,2 Dichloroethane exhibits similar behavior going to 1.4882 in Figure 5-4 from 1.5488 in Figure 5-2. The proximity of the slopes for both diluents with pure water and the duel acid system seems to substantiate the conclusion that the introduction of the acids does not affect equilibrium water content significantly. 


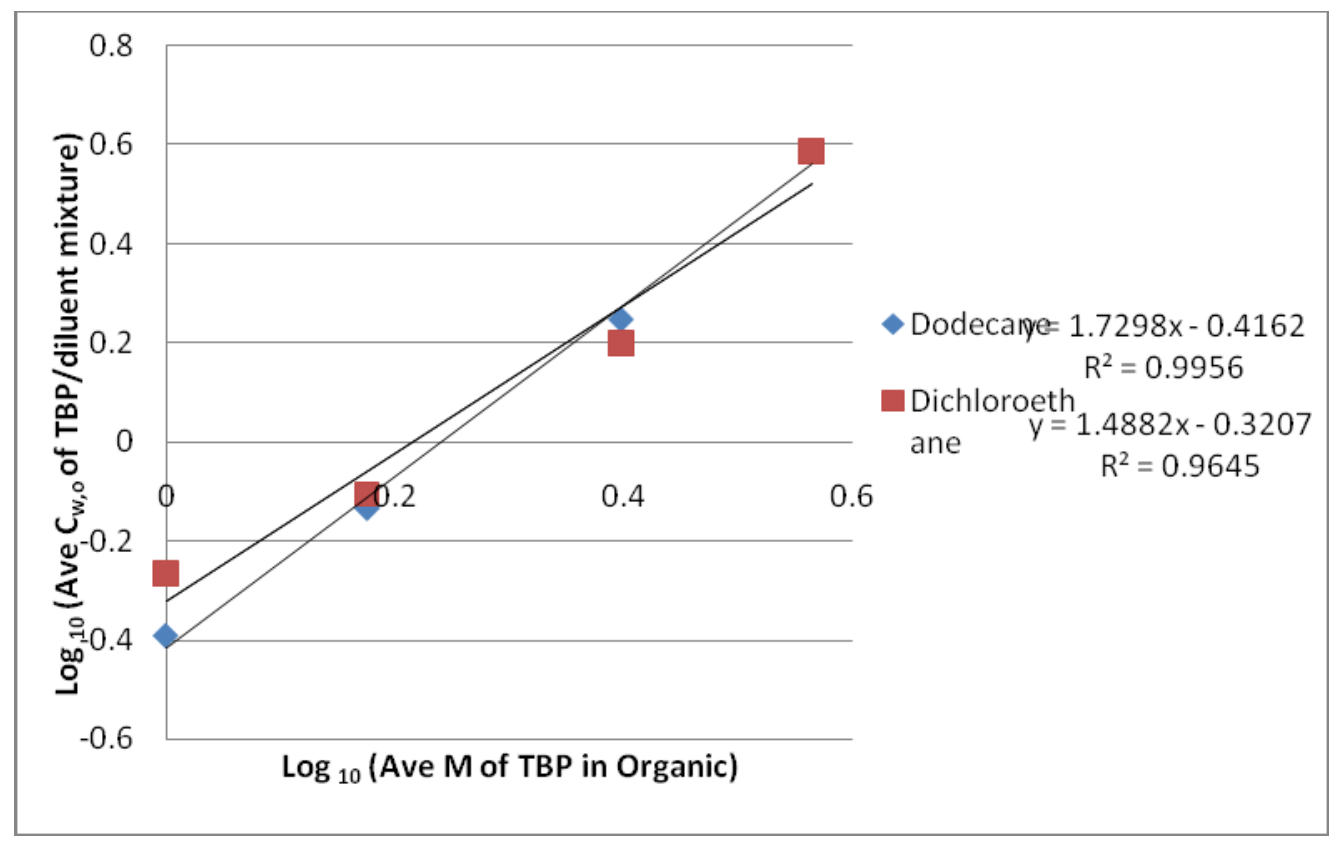

Figure 5-4. Average CW,O of TBP/diluent solutions in the pressence of duel acid system

To understand any connections between water content in the duel acid system and the extraction of acetic acid and nitric acid, a comparison was performed for the slopes of the $\log -$ $\log$ plots for the water content with n-Dodecane to the equilibrium acid extraction data given in Table 5-1 and Figure 5-5 for the approximately $0.25 \mathrm{M}$ nitric acid and $0.025 \mathrm{M}$ acetic acid mixture. Equilibrium acid extraction data is taken from Mitchell et al. $\mathrm{K}_{\mathrm{D}}$ is defined as the $\mathrm{C}_{\text {acid,organic }} / \mathrm{C}_{\text {acid,aqueous. }}$

Table 5-1. Equilibrium distribution data for acetic and nitric acid in varying concentrations of TBP in n-Dodecane

\begin{tabular}{|c|c|c|c|c|}
\hline AQUEOUS SYSTEM & ORGANIC SYSTEM & $\mathrm{K}_{\mathrm{D}}$ Nitric Acid & $\mathrm{K}_{\mathrm{D}}$ Acetic Acid & Separation Factor \\
\hline Approximately & $\mathrm{n}$-Dodecane & 0.022 & -0.021 & $\mathrm{~N} / \mathrm{A}$ \\
\hline
\end{tabular}




\begin{tabular}{|c|c|c|c|c|}
\hline 0.25M Nitric Acid \\
$0.025 \mathrm{M}$ Acetic Acid \\
$\begin{array}{c}\text { and } \\
\text { Water }\end{array}$ & $1 \mathrm{M}$ TBP in dodecane & 0.049 & 0.183 & 3.735 \\
\cline { 2 - 5 } & 1.5M TBP-dodecane & 0.098 & 0.878 & 8.959 \\
\cline { 2 - 5 } & $2.5 \mathrm{M}$ TBP-dodecane & 0.308 & 1.481 & 4.808 \\
\cline { 2 - 5 } & TBP & 0.620 & 2.573 & 4.150 \\
\hline
\end{tabular}

It is important to remember that the acetic acid is at $1 / 10$ the concentration of the nitric acid. Even though, the $K_{D}$ values for acetic acid are higher than those for nitric acid, the overall amount of nitric acid extracted may be greater than the amount of acetic acid extracted.

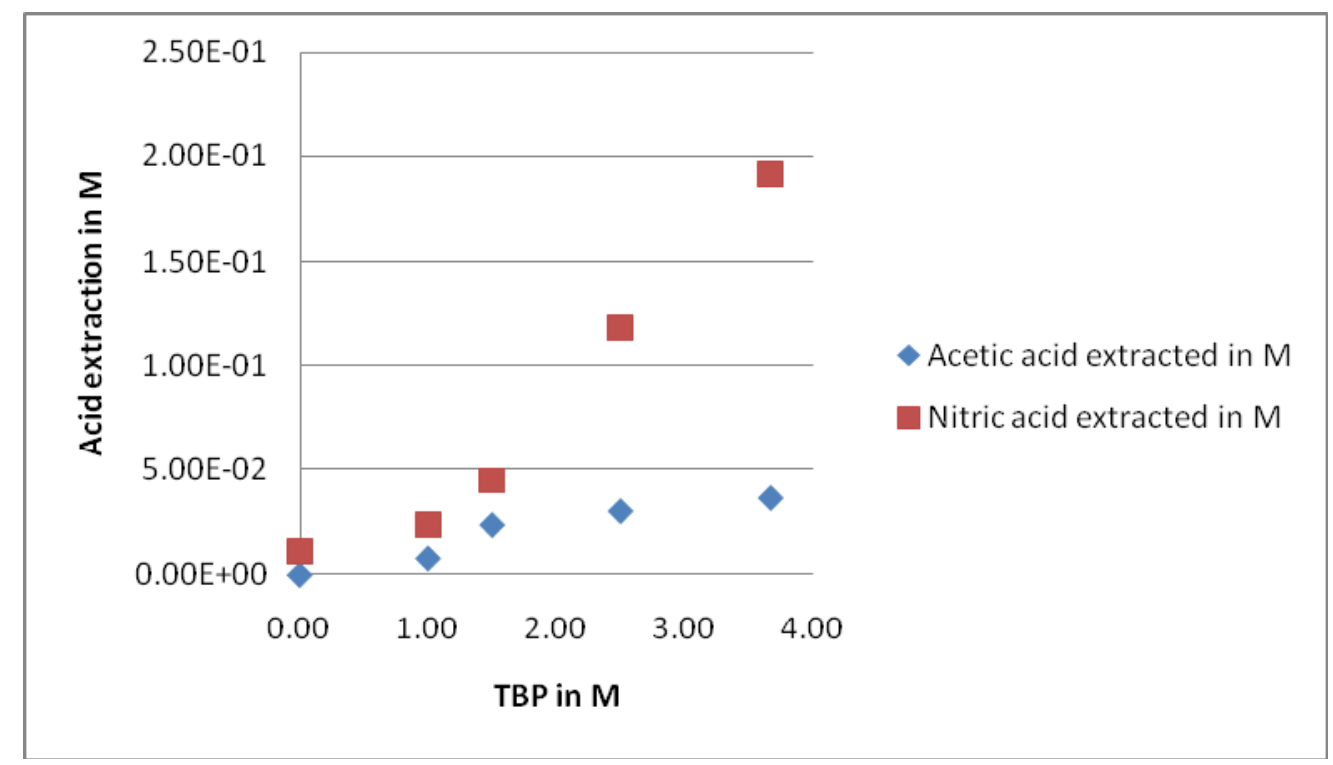

Figure 5-5. Acid extraction data for acetic acid and nitric acid into a TBP/n-Dodecane solution 


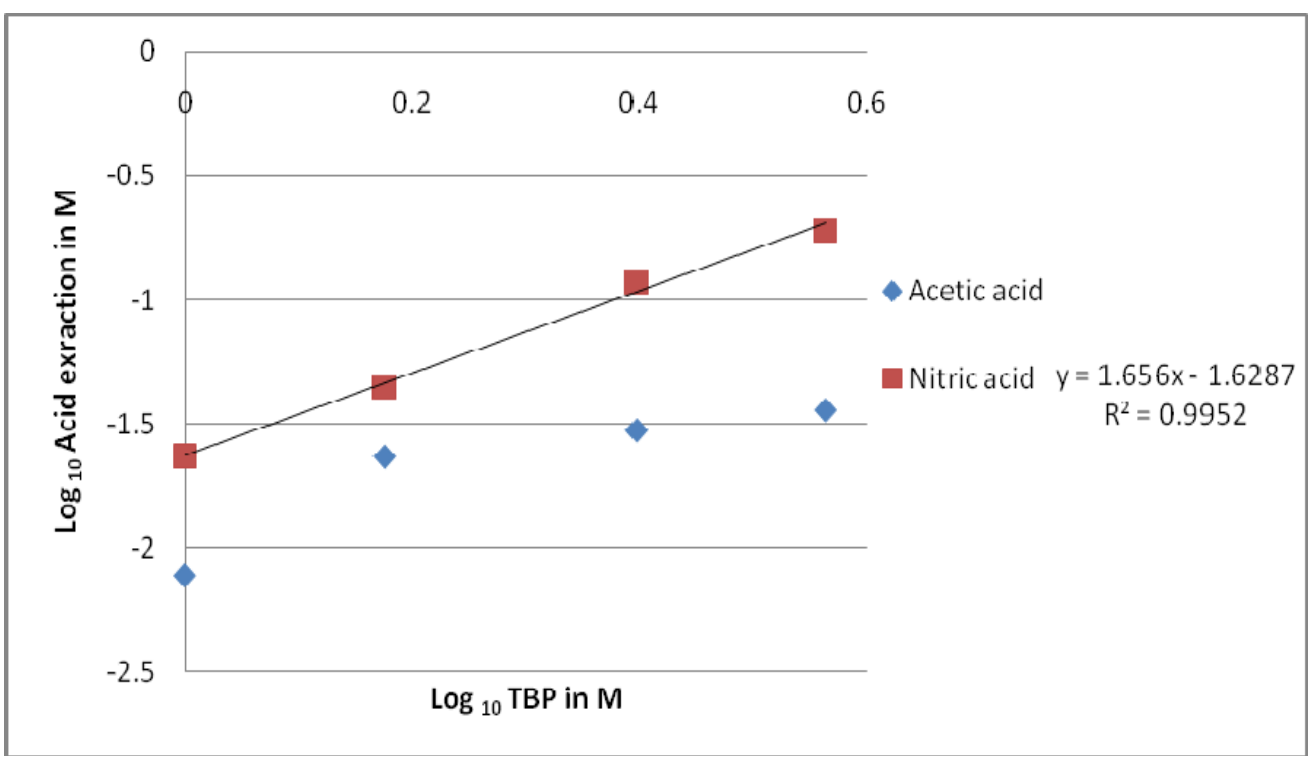

Figure 5-6. Log-log plot of acid extraction data for acetic acid and nitric acid into TBP/nDodecane solution

After studying the log-log plot in Figure 5-6, it seems that, while the nitric acid is not extracted to the same extent as water, the nitric acid extraction into the TBP/ n-Dodecane mixture follows a similar mechanism to that of water absorption from the duel acid mixture in Figure 5-3. The log-log slope for the nitric acid extraction in Figure 5-6 is 1.656 which is close to the 1.4882 reported for the water content of the TBP/n-Dodecane mixture with an acid based aqueous phase in Figure 5-4.

Analysis of the acetic acid extraction data in Figure 5-6 appears to show a limit to acetic acid extraction just under 0.05 . 


\section{Conclusions}

The data suggests that the water content of the organic mixture after contact is largely independent of the diluent used; all three diluents seem to provide similar results. The addition of equal volumes of, $0.5 \mathrm{M}$ nitric acid and $0.05 \mathrm{M}$ acetic acid resulting in an aqueous phase of approximately $0.25 \mathrm{M}$ nitric acid and $0.025 \mathrm{M}$ acetic acid also seems to have minimal affect on the amount of water absorbed at equilibrium; the slopes of the log-log plots compare favorably between the pure water and acid based aqueous solutions. This consistency in water absorption seems to point to an independence from any acid co-extraction, and the range of slopes between 1.5 and 2 for all samples points to a combination of $[\mathrm{TBP}]_{2}\left[\mathrm{H}_{2} \mathrm{O}\right]_{3}$ to $[\mathrm{TBP}]_{1}\left[\mathrm{H}_{2} \mathrm{O}\right]_{2}$ hydration complexes.

Comparison of the acid extraction to water content in the TBP/n-Dodecane system tends to point to a similar extraction behavior for water and nitric acid. While the nitric acid is not extracted in the same quantity as water, comparison of the log-log plots for water content and nitric acid extraction points to the similarity of slopes for their linear regressions; nitric acid has a $\log$-log slope of 1.656 and water has a log-log slope of 1.4882 . This seems to suggest that there might be a similar mechanism for the equilibrium acid extraction and water content.

\section{Acknowledgement}

The authors thank Joseph Birdwell, Jessica Mitchell, Christina Karni, and Jon Garrison for their support, advice and hard work during the course of this study. This research was supported by the U.S. Department of Energy, NERI program, under DOE Contract No. DEPS07-05ID14713. 


\section{Literature Cited}

(1) Mitchell, J.; Watson, J.S.; Johnson, J; Spencer, B.B.; Counce, R.M.; Del Cul, G.D. Extracting Acetic Acid from Acidic Solutions. Separation Science and Technology. 2008. 43(9). 2537.

(2) Damiani, L.; Fattore, V. Nitric acid, water and tributylphosphate equilibrium system. energia nucleare. 1959. 6(12). 793.

(3) Davis Jr. W.; Mrohek, J.; Hardy, C.J. The System: Tri-n-butyl phosphate

(TBP)-nitric acid-water-I Activities of TBP in equilibrium with aqueous nitric acid and partial molar volumes of the three components in the TBP phase. Journal of Inorganic and Nuclear Chemistry.1966. 28. 2001.

(4) Alcock, K.; Grimley, S.S.; Healy, T.V.; Kennedy, J.; McKay, H.A.C. The extraction of nitrates by tri-n-butyl phosphate; part I.-the system TBP + diluent $+\mathrm{H}_{2} \mathrm{O}+\mathrm{HNO}_{3}$. Trans. Faraday Society. 1956. 52. 39.

(5) Blaylock, C.R.; Tedder, D.W. Competitive Equilibria in the System: Water, Nitric Acid, Trin-Butyl Phosphate, and Amsco 125-82. Solvent Extraction in Ion Exchange. 1988. 7(2). 249.

(6) Hardy, C.J.; Fairhurst, D.; McKay H.A.C.; Willson, A.M. Extraction of Water by Tri-n-butyl Phosphate. Transactions of the Faraday Society. 1964. 60(501). 1626.

(7) Friehmelt, V.; He, A.; Yang, Z.; Marx, G. Transport behavior of water and nitric acid in tributyl phosphate/n-dodecane mixtures. Analytica Chimica Acta (1987), 201. 135.

(8) Roddy, J.W. Interactions in the Tri-n-butyl phosphate-water-diluent system. Journal of Inorganic and Nuclear Chemistry. 1978. 40(10). 1787. 
(9) Pushlenkov, M.F.; Komarov, E.V.; Shurenkova M.E.; Intermolecular interaction in the system; tri-n-buytl-phosphate-nitric acid-water. Journal of Structural Chemistry. 1961. 2(6). 1573.

(10) Shatzberg, Paul. Solubilities of Water in Several Normal Alkanes From $\mathrm{C}_{7}$ to $\mathrm{C}_{16}$. Journal of Physical Chemisty. 1963. 67. 776-779.

(11) Spencer, Barry B. Thermodynamics of the Extraction of Uranium from Strong Aqeuous Electrolyte Solutions. Doctoral Dissertation, University of Tennessee. 1994. 68. 


\section{Appendix A}

Water Solubility $\left(\mathrm{C}_{\mathrm{w}, \mathrm{o}}\right)$ Results from Contact Between Deionized Water and TBP-Organic

Diluent Mixtures

Table 5-2. Water solubility of TBP/n-Dodecane mixture

\begin{tabular}{|c|c|c|}
\hline TBP in $M$ & $\mathrm{C}_{\mathrm{w}, 0}$ of Dodecane mixture in $\mathrm{L}_{\text {water }} / \mathrm{L}_{\text {organic }}$ & $\mathrm{C}_{\mathrm{w}, \mathrm{o}}$ in $\mathrm{M}_{\text {water }}$ \\
\hline 0.00 & $5.00 \mathrm{E}-05$ & $2.78 \mathrm{E}-03$ \\
\hline 0.00 & $4.50 \mathrm{E}-05$ & $2.50 \mathrm{E}-03$ \\
\hline 0.94 & $6.67 \mathrm{E}-03$ & $3.71 \mathrm{E}-01$ \\
\hline 0.94 & $6.48 \mathrm{E}-03$ & $3.60 \mathrm{E}-01$ \\
\hline 0.94 & $6.57 \mathrm{E}-03$ & $3.65 \mathrm{E}-01$ \\
\hline 1.50 & $1.36 \mathrm{E}-02$ & $7.55 \mathrm{E}-01$ \\
\hline 1.50 & $1.31 \mathrm{E}-02$ & $7.29 \mathrm{E}-01$ \\
\hline 1.50 & $1.33 \mathrm{E}-02$ & $7.38 \mathrm{E}-01$ \\
\hline 2.50 & $3.22 \mathrm{E}-02$ & $1.79 \mathrm{E}+00$ \\
\hline 2.50 & $3.25 \mathrm{E}-02$ & $1.81 \mathrm{E}+00$ \\
\hline 2.50 & $3.24 \mathrm{E}-02$ & $1.80 \mathrm{E}+00$ \\
\hline 2.50 & $3.22 \mathrm{E}-02$ & $1.79 \mathrm{E}+00$ \\
\hline 3.67 & $6.48 \mathrm{E}-02$ & $3.60 \mathrm{E}+00$ \\
\hline 3.67 & $6.48 \mathrm{E}-02$ & $3.60 \mathrm{E}+00$ \\
\hline 3.67 & $6.48 \mathrm{E}-02$ & $3.60 \mathrm{E}+00$ \\
\hline
\end{tabular}

Table 5-3. Water solubility of TBP/1,2 Dichloroethane mixture

\begin{tabular}{|c|c|c|}
\hline TBP in $\mathrm{M}$ & $\mathrm{C}_{\mathrm{w}, \mathrm{o}}$ of 1,2 Dichloroethane mixture in $\mathrm{L}_{\text {water }} / \mathrm{L}_{\text {organic }}$ & $\mathrm{C}_{\mathrm{w}, \mathrm{o}}$ in $\mathrm{M}_{\text {water }}$ \\
\hline 0.00 & $2.37 \mathrm{E}-03$ & $1.31 \mathrm{E}-01$ \\
\hline 0.00 & $2.33 \mathrm{E}-03$ & $1.30 \mathrm{E}-01$ \\
\hline 0.94 & $8.37 \mathrm{E}-03$ & $4.65 \mathrm{E}-01$ \\
\hline 0.94 & $8.27 \mathrm{E}-03$ & $4.60 \mathrm{E}-01$ \\
\hline 0.94 & $8.27 \mathrm{E}-03$ & $4.60 \mathrm{E}-01$ \\
\hline 1.50 & $1.35 \mathrm{E}-02$ & $7.51 \mathrm{E}-01$ \\
\hline 1.50 & $1.35 \mathrm{E}-02$ & $7.51 \mathrm{E}-01$ \\
\hline 1.50 & $1.37 \mathrm{E}-02$ & $7.60 \mathrm{E}-01$ \\
\hline 2.49 & $3.46 \mathrm{E}-02$ & $1.92 \mathrm{E}+00$ \\
\hline 2.49 & $3.48 \mathrm{E}-02$ & $1.93 \mathrm{E}+00$ \\
\hline 2.49 & $3.43 \mathrm{E}-02$ & $1.90 \mathrm{E}+00$ \\
\hline 2.49 & $3.51 \mathrm{E}-02$ & $1.95 \mathrm{E}+00$ \\
\hline 3.67 & $6.48 \mathrm{E}-02$ & $3.60 \mathrm{E}+00$ \\
\hline 3.67 & $6.48 \mathrm{E}-02$ & $3.60 \mathrm{E}+00$ \\
\hline 3.67 & $6.48 \mathrm{E}-02$ & $3.60 \mathrm{E}+00$ \\
\hline
\end{tabular}


Table 5-4. Water solubility of TBP/FS-13 mixture

\begin{tabular}{|c|c|c|}
\hline TBP in $M$ & $\mathrm{C}_{\mathrm{w}, 0}$ of FS-13 mixture in $\mathrm{L}_{\text {water }} / \mathrm{L}_{\text {organic }}$ & $\mathrm{C}_{\mathrm{w}, \mathrm{O}}$ in $\mathrm{M}_{\text {water }}$ \\
\hline 0.00 & $2.78 \mathrm{E}-03$ & $1.54 \mathrm{E}-01$ \\
\hline 0.00 & $2.59 \mathrm{E}-03$ & $1.44 \mathrm{E}-01$ \\
\hline 0.00 & $2.69 \mathrm{E}-03$ & $1.49 \mathrm{E}-01$ \\
\hline 0.93 & $8.75 \mathrm{E}-03$ & $4.86 \mathrm{E}-01$ \\
\hline 0.93 & $8.66 \mathrm{E}-03$ & $4.81 \mathrm{E}-01$ \\
\hline 0.93 & $8.85 \mathrm{E}-03$ & $4.92 \mathrm{E}-01$ \\
\hline 1.48 & $1.41 \mathrm{E}-02$ & $7.81 \mathrm{E}-01$ \\
\hline 1.48 & $1.41 \mathrm{E}-02$ & $7.86 \mathrm{E}-01$ \\
\hline 1.48 & $1.41 \mathrm{E}-02$ & $7.81 \mathrm{E}-01$ \\
\hline 2.41 & $3.23 \mathrm{E}-02$ & $1.79 \mathrm{E}+00$ \\
\hline 2.42 & $3.18 \mathrm{E}-02$ & $1.76 \mathrm{E}+00$ \\
\hline 2.41 & $3.19 \mathrm{E}-02$ & $1.77 \mathrm{E}+00$ \\
\hline 3.45 & $6.48 \mathrm{E}-02$ & $3.60 \mathrm{E}+00$ \\
\hline 3.45 & $6.48 \mathrm{E}-02$ & $3.60 \mathrm{E}+00$ \\
\hline 3.45 & $6.48 \mathrm{E}-02$ & $3.60 \mathrm{E}+00$ \\
\hline
\end{tabular}




\section{Appendix B}

Water Solubility $\left(\mathrm{C}_{\mathrm{w}, \mathrm{o}}\right)$ Results after Contact with De-Ionized Water containing approximately 0.25 M Nitric Acid and 0.025 M Acetic with Various Concentrations of TBP in Organic Diluents

Table 5-5. Water solubility of TBP/n-Dodecane mixture in the presence of the duel acid system

\begin{tabular}{|c|c|c|}
\hline$M$ of TBP & $\mathrm{C}_{\mathrm{w}, \mathrm{O}}$ of Dodecane mixture in $\mathrm{L}_{\text {water }} / \mathrm{L}_{\text {organic }}$ & $\mathrm{C}_{\mathrm{w}, \mathrm{0}}$ in $\mathrm{M}_{\text {water }}$ \\
\hline 0.00 & $2.71 \mathrm{E}-04$ & $1.51 \mathrm{E}-02$ \\
\hline 0.00 & $2.26 \mathrm{E}-04$ & $1.26 \mathrm{E}-02$ \\
\hline 0.00 & $1.36 \mathrm{E}-04$ & $7.54 \mathrm{E}-03$ \\
\hline 1.00 & $7.26 \mathrm{E}-03$ & $4.03 \mathrm{E}-01$ \\
\hline 1.00 & $7.36 \mathrm{E}-03$ & $4.09 \mathrm{E}-01$ \\
\hline 1.00 & $7.26 \mathrm{E}-03$ & $4.03 \mathrm{E}-01$ \\
\hline 1.50 & $1.35 \mathrm{E}-02$ & $7.49 \mathrm{E}-01$ \\
\hline 1.50 & $1.30 \mathrm{E}-02$ & $7.24 \mathrm{E}-01$ \\
\hline 1.50 & $1.32 \mathrm{E}-02$ & $7.34 \mathrm{E}-01$ \\
\hline 1.50 & $1.30 \mathrm{E}-02$ & $7.24 \mathrm{E}-01$ \\
\hline 2.50 & $3.15 \mathrm{E}-02$ & $1.75 \mathrm{E}+00$ \\
\hline 2.50 & $3.20 \mathrm{E}-02$ & $1.78 \mathrm{E}+00$ \\
\hline 2.50 & $3.17 \mathrm{E}-02$ & $1.76 \mathrm{E}+00$ \\
\hline 2.50 & $3.17 \mathrm{E}-02$ & $1.76 \mathrm{E}+00$ \\
\hline 3.67 & $7.05 \mathrm{E}-02$ & $3.92 \mathrm{E}+00$ \\
\hline 3.67 & $6.85 \mathrm{E}-02$ & $3.81 \mathrm{E}+00$ \\
\hline 3.67 & $6.92 \mathrm{E}-02$ & $3.84 \mathrm{E}+00$ \\
\hline
\end{tabular}


Table 5-6. Water solubility of TBP/1,2 Dichloroethane mixture in the presence of the duel acid system

\begin{tabular}{|c|c|c|}
\hline$M$ of TBP & $C_{w, 0}$ of 1,2 Dichloroethane mixture in $L_{\text {water }} / L_{\text {organic }}$ & $C_{w, 0}$ in $M_{\text {water }}$ \\
\hline 0.00 & $3.08 \mathrm{E}-03$ & $1.71 \mathrm{E}-01$ \\
\hline 0.00 & $2.71 \mathrm{E}-03$ & $1.51 \mathrm{E}-01$ \\
\hline 0.00 & $2.71 \mathrm{E}-03$ & $1.51 \mathrm{E}-01$ \\
\hline 1.00 & $9.15 \mathrm{E}-03$ & $5.08 \mathrm{E}-01$ \\
\hline 1.00 & $9.70 \mathrm{E}-03$ & $5.39 \mathrm{E}-01$ \\
\hline 1.00 & $9.97 \mathrm{E}-03$ & $5.54 \mathrm{E}-01$ \\
\hline 1.00 & $9.70 \mathrm{E}-03$ & $5.39 \mathrm{E}-01$ \\
\hline 1.00 & $9.60 \mathrm{E}-03$ & $5.34 \mathrm{E}-01$ \\
\hline 1.50 & $1.41 \mathrm{E}-02$ & $7.85 \mathrm{E}-01$ \\
\hline 1.50 & $1.39 \mathrm{E}-02$ & $7.70 \mathrm{E}-01$ \\
\hline 1.50 & $1.41 \mathrm{E}-02$ & $7.85 \mathrm{E}-01$ \\
\hline 1.50 & $1.43 \mathrm{E}-02$ & $7.96 \mathrm{E}-01$ \\
\hline 2.50 & $2.83 \mathrm{E}-02$ & $1.57 \mathrm{E}+00$ \\
\hline 2.50 & $2.85 \mathrm{E}-02$ & $1.59 \mathrm{E}+00$ \\
\hline 2.50 & $2.84 \mathrm{E}-02$ & $1.58 \mathrm{E}+00$ \\
\hline 3.67 & $7.05 \mathrm{E}-02$ & $3.92 \mathrm{E}+00$ \\
\hline 3.67 & $6.85 \mathrm{E}-02$ & $3.81 \mathrm{E}+00$ \\
\hline 3.67 & $6.92 \mathrm{E}-02$ & $3.84 \mathrm{E}+00$ \\
\hline
\end{tabular}




\section{Chapter VI: Evaluation of Centrifugal Contactors for the Extraction of Acetic Acid from UREX+ Streams}




\section{Abstract}

In the UREX+ process, acetohydroxamic acid (AHA) is utilized to inhibit the extraction of plutonium in the initial UREX step. (AHA) then breaks down to produce acetic acid which needs to be removed from the aqueous stream in order to facilitate the recycle of nitric acid.

This study investigates the use of annular centrifugal contactors for the liquid-liquid extraction of acetic acid into an organic phase consisting of 1.5M TBP in n-Dodecane.

Initial break time tests were performed in order to investigate the mixing/separation viability of the organic/aqueous system, and after determining that centrifugal contactors could be used to perform the liquid-liquid extraction, hydraulic curves were created to establish the combination of rotation rate and flow rates and flow rates which should be used to insure proper separation of the two outlet phases. Finally, extraction efficiency data was collected to examine the system conditions that provided the most efficient removal of acetic acid. 


\section{Introduction}

The objective of this project was to evaluate the mass transfer performance of an annular centrifugal contactor for the extraction of acetic acid from an acid based UREX+ stream. The solvent chosen for the study was $1.5 \mathrm{M}$ TBP in n-Dodecane. Previous work has shown that this solvent showed the ability to effectively extract acetic acid from a mixture of acetic acid and nitric acid. The aqueous phase used to simulate the acid based UREX+ stream was an equal molar combination of $1 \mathrm{M}$ nitric acid and $0.1 \mathrm{M}$ acetic acid. ${ }^{(3)}$

Removal of acetic acid from the UREX+ process is important as the presence of acetic acid is thought to interfere with the recycle of the nitric acid. Acetic acid is formed through the thermo chemical breakdown of AHA, acetohydroxamic acid which is used in the UREX+ process to suppress the co-extraction of plutonium during the initial UREX step.

Determination of mass transfer performance was broken down into three steps. The first was determination of system feasibility in industrial mixer settlers through determination of the

unitless dispersion number. ${ }^{(2)}$ The second step was investigation of the appropriate RPM and total throughput ranges for effective mixing and separation through construction of hydraulic performance curves. Finally, mass transfer efficiencies were calculated through analysis of equilibrium acid distributions to those gathered after one pass through the contactor. 


\section{Equipment}

The contactor used in the study was a Costner Industries version 02 annular centrifugal contactor with some slight adjustments. The curved vein bottom plate and attached separator housing were removed from the original equipment and replaced with a straight vein bottom plate which promoted better mixing. ${ }^{(3)}$

The separator housing does not allow a portion of the liquid to access the mixing vein and therefore drastically reduces mass transfer in the mixing zone.

The straight veined bottom plate was chosen to replace the curved vein plate, evidently, the removal of the separator housing increased overall mass transfer. In addition, the straight vein plate has been shown to promote better mixing than the curved plate. ${ }^{(3)}$

The V-02 from CINC come equipped with a 5-cm rotor, a 0.13-H.P. motor with a max rpm of 6000 and a listed throughput capacity of $1.9 \mathrm{~L} / \mathrm{m}$. The system throughput capacity is not a static number and will vary with system conditions: O/A ratio, viscosity, limits on degree of separation etc... For this reason, no real significance was attributed to the $1.9 \mathrm{~L} / \mathrm{m}$ limit.

Both the aqueous and organic phases were pumped into the contactor with a ColeParmer ${ }^{\circledR}$ Consol Digital Dispensing Drive with Remote Control using Micropump® A-mount Pump Heads, 115 VAC. Collection vessels were two liter Pyrex jars and the lines were polypropylene. 
The acetic and nitric acids were purchased from Fischer Chemicals and were used as is without pretreatment. The n-dodecane and TBP were purchased from both Fischer Chemicals and Acros Organics and were also used as is, without pretreatment. There was no noticeable difference in performance between the Acros and Fischer Chemicals organics. Careful attention was paid to making sure consistency was present across an entire test. If Fischer Chemicals were used in the first part of an individual test, they were used throughout the entire test.

Titrations were performed using a 765 Metrohm Dosimat titration dispenser and a Mettler Toledo Seveneasy $\mathrm{pH}$ meter, and the $\mathrm{NaOH}$ utilized for titration of the acids was standardized with HCL provided from Fischer Chemicals. 


\section{Procedures}

\subsection{Break-time Tests}

The unitless dispersion number has long been used to determine the suitability of an organic/aqueous system for separation in a mixer settler. Here, it has been applied to resolve the suitability of the $1.5 \mathrm{M}$ TBP in n-Dodecane system for separation in an annular centrifugal contactor under the following equation

$$
N_{D_{i}}=\frac{1}{t_{B}} \sqrt{\frac{\Delta z}{g}}
$$

where $t_{B}$ is the time for the dispersion band to break in seconds, $\Delta z$ is the total height of the fluid in $\mathrm{cm}$, and $\mathrm{g}$ is acceleration of gravity in $\mathrm{cm} / \mathrm{s}^{2}$. (2)

Break tests were performed with three different organic to aqueous ratios: 1:1, 1:2 and 2:1 and executed in one hundred $\mathrm{ml}$ graduated cylinders while time was kept with a stop watch. Being careful to avoid premature mixing, the heavier of the two phases, the aqueous phase, was poured into the cylinder first followed by the organic phase, and the cylinder was then capped with a glass stopper. The mixture was then shaken vigorously for ten seconds, held still for ten seconds and then shaken again for another ten second period. Immediately after shaking, the graduated cylinder was placed on a flat surface, and the time for the dispersion band to break was 
recorded. To assist in the determination of the continuous phase, original and final interface positions were also recorded as well as the relative cloudiness of both phases after mixing.

\subsection{Hydraulic Tests}

Hydraulic Tests were done to locate the minimum rpm required to separate the two phase system at a given total throughput and organic to aqueous ratio. Here, acetic acid was neglected and the aqueous phase consisted of only $0.5 \mathrm{M}$ nitric acid while the organic phase was maintained at $1.5 \mathrm{M}$ TBP in $\mathrm{n}$-Dodecane. The heavy phase pump was started first, allowing a wall of the aqueous fluid to build up inside the separation zone of the contactor. Once the heavy phase began to exit the contactor, the light phase pump was started. As soon as the light phase began to exit the light phase outlet, a stop watch was started and a total of at least three minutes was allowed for the contactor to reach steady state. Using plastic disposable centrifuge tubes, $\sim 20 \mathrm{ml}$ samples of both the aqueous and organic outlets were taken. The two samples were marked and then set aside. The rotor rpm were then reduced by a factor of 100 , and the stop watch was reset, allowing another three minutes for the contactor to again reach steady state before the next sample was collected. Samples were taken in this manner for many different flow rates across multiple organic to aqueous ratios. The samples were left overnight and examined the next day to check for cross contamination of the aqueous phase into the organic 
outlet or vice-versa. The aqueous sample was examined for a significant layer of organic lying on top the aqueous phase, and the organic sample was examined for aqueous phase bubble formation at the bottom of the tube. While, aqueous contamination of the organic phase was never discovered, organic phase contamination of the aqueous outlet was prevalent in certain samples. Anything above $1 \%$ volume cross contamination was considered unacceptable, and the procedure for determining the contamination level is outlined below.

The diameter of the aqueous bubble was measured in centimeters. Assuming a spherical bubble, the volume of the bubble was calculated from the diameter, and its percentage of total volume determined. Fail points, points where contamination was greater than $1 \%$, were charted and graphed for organic to aqueous ratios of $1: 1,1.2: 1,1.4: 1,2: 1$ providing hydraulic curves for use in the extraction testing that follows. Operation above the line is considered possible, but operation below the line could lead to cross-contamination and skewed extraction results.

A 1:2 organic to aqueous ratio was also tested. However, the 0.95 in weir did not provide a single non-contaminated point regardless of throughput/rpm combination chosen. Therefore, the weir size was adjusted to 1.0 inch and the hydraulic tests were rerun. While we were able to find ranges where the system would effectively separate the two phases, location of a single fail point was unsuccessful.

To decrease the cost associated with the organic phase chemicals, the 1.5M TBP in nDodecane was recycled. Any amount of nitric acid extracted into the organic phase was 
considered negligible in relation to the overall mass of the organic phase and was assumed to not affect overall hydraulic performance.

\subsection{Extraction Test}

Extraction tests were performed to evaluate the efficiency with which the mixing and holdup time of the contactor allowed for extraction of acetic and nitric acid from the aqueous phase stream by the organic phase stream. Efficiency is defined as follows for acetic acid

$$
\begin{aligned}
& \text { Efficiency }_{\text {Acetic Acid }}=\frac{\mathrm{K}_{\mathrm{D}_{\text {Contactor }}}^{\prime}}{\mathrm{K}_{\mathrm{D}_{\text {Equilibrium }}}} \\
& \text { where } \\
& \mathrm{K}_{\mathrm{D}_{\text {Contactor }}}^{\prime}=\frac{\left[\text { Acetic } \text { Acid }_{\text {Extract }}\right]}{\left[\text { Acetic } \text { Acid }_{\text {Raffinate }}\right]} \\
& \text { and } \\
& \mathrm{K}_{\mathrm{D}_{\text {Equilibrium }}}=\frac{\left[\text { Acetic } \text { Acid }_{\text {Equilibrium Organic }}\right]}{\left[\text { Acetic } \text { Acid }_{\text {Equilibrium Aqueous }}\right]} \text {. }
\end{aligned}
$$

The equations for the efficiency of nitric acid extraction are analogous. 
Initially a solution was prepared of $0.5 \mathrm{M}$ nitric acid and $0.05 \mathrm{M}$ acetic acid, followed by a solution of 1.0 M NaOH. The sodium hydroxide solution was standardized with a known standard of $0.5 \mathrm{M} \mathrm{HCl}$. The standardized $1.0 \mathrm{M} \mathrm{NaOH}$ was then used to titrate the $0.5 \mathrm{M}$ nitric acid and $0.05 \mathrm{M}$ acetic acid solution in order to determine exact concentrations used in the contactor feed and equilibrium control tests. The equilibrium control was prepared in order to test the extraction efficiency of the contactor in comparison to equilibrium as follows: $100 \mathrm{ml}$ of the standardized dual acid solution and $100 \mathrm{ml}$ of $1.5 \mathrm{M}$ TBP in $\mathrm{n}$-dodecane was mixed and vigorously agitated in a $250 \mathrm{ml}$ separatory funnel for approximately five minutes. The funnel was placed in a ring stand were the mixture was allowed to separate and reach equilibrium. After which time, the bottom aqueous phase was drawn off and titrated with the above standardized $1.0 \mathrm{M} \mathrm{NaOH}$.

As nitric acid disassociates to a far greater extent than acetic acid in the dual acid system, the first equilibrium point of the titration curve is taken as the equivalence point for the nitric acid. The difference between the first inflection point and the second inflection point is taken as the volume of $\mathrm{NaOH}$ needed to neutralize acetic acid.

The same TBP in n-dodecane and standardized aqueous used in the equilibration test were fed through the contactor at a chosen organic to aqueous ratio. Both the organic and aqueous solutions were pumped into the contactor at flow rates corresponding to the points chosen for testing from the hydraulic curves. The extraction testing utilized the same pumps, 
weir size and bottom plate as described in the contactor design. The contactor was set at an RPM rate exactly 100 revolutions per minute above the hydraulic curve for the point selected to ensure proper separation of the outlet phases. Samples of the raffinate (aqueous stream outlet) were taken at multiple times until at least one of the inlet tanks had pumped its entire volume through the contactor. The same procedure used to analyze the equilibrium control was used to analyze the samples from the contactor. Once the concentrations of nitric and acetic acid were determined from the raffinate samples, the amounts of nitric and acetic acid extracted into the organic phase were determined by subtracting the average of the raffinate concentrations from the feed concentrations. The series of calculations below describe the derivation of the values for acetic acid which were then used to determine contactor efficiency. Similar calculations were made for nitric acid as well. Efficiencies were tested at two different points along the hydraulic curve for organic to aqueous ratios of both 1:1 and 2:1.

$$
\begin{aligned}
& {\left[\text { Nitric Acid }_{\text {Feed }}\right]=[\mathrm{NaOH}] \frac{\mathrm{V}_{\text {NaOH }_{\text {Nitic equivelane }}}}{\mathrm{V}_{\text {Total Aqueous Sample }}}} \\
& {[\text { Acetic Acid } \text { Feed }]=[\mathrm{NaOH}] \frac{\left(\mathrm{V}_{\mathrm{NaOH}_{\text {Acetic cauivelance }}}-\mathrm{V}_{\mathrm{NaOH}_{\text {Niric equivelance }}}\right)}{\mathrm{V}_{\text {Total Feed Sample }}}} \\
& {[\text { Acetic Acid } \text { Equilibrium Aqueous }]=[\mathrm{NaOH}] \frac{\left(\mathrm{V}_{\mathrm{NaOH}_{\text {Aectic equivelanee }}}-\mathrm{V}_{\text {NaOH }} \mathrm{V}_{\text {Niric equivelanee }}\right)}{\mathrm{V}_{\text {Total Aqueous Equilibrium Sample }}}} \\
& {[\text { Acetic Acid } \text { Equilibrium Organic }]=[\text { Acetic Acid } \text { Feed }]-\left[\text { Acetic Acid }_{\text {Equilibrium Aqueous }}\right]}
\end{aligned}
$$




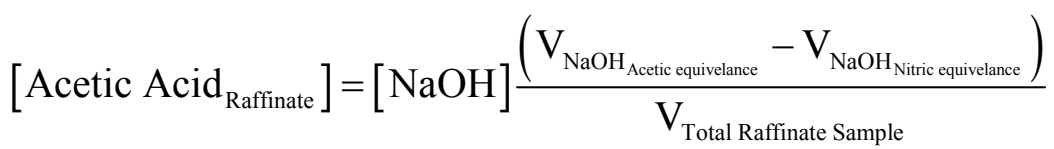

$$
\begin{aligned}
& {\left[\text { Acetic } \operatorname{Acid}_{\text {Extract }}\right]=\left[\text { Acetic Acid }_{\text {Feed }}\right]-\left[\text { Acetic Acid }_{\text {Raffinate }}\right]}
\end{aligned}
$$

Again the 1:2 organic to aqueous ratio was tested with a different weir size than the other ratios. As no exact fail point was ever calculated for the 1:2 ratio, no hydraulic curve was ever generated, and the two rpm and throughput combinations for testing of contactor efficiency were chosen from the ranges that provided proper separation with the 1.00 inch weir.

\section{Results}

\subsection{Break-time Test}

(Table given in Appendix 1)

Break-time tests were performed as described previously and the results are given in Appendix 1, Table 6-1. Each test was performed twice to insure accurate results.

\section{1:1 Organic/Aqueous Ratio}

After shaking the cylinder and starting the timer, it was noticed that bubbles were escaping from the top of the dispersion band as it shrank down the height of the cylinder. No bubbles formed in the bottom, aqueous layer, but entrainment and bubbles were evident in the top, organic, phase. There was also noticeable cloudiness of the organic phase while the aqueous phase remained clear. The dispersion numbers of $1.31 * 10^{-3}$ and $1.33 * 10^{-3}$ dictate that the mixture is suitable for introduction into mixer settlers, and the lack of entrainment or cloudiness in the 
aqueous phase indicates an organic continuous phase. This is consistent with the results reported from Ralph Leonard in his interactions with a similar system. ${ }^{(2)}$

\section{2:1 Organic/Aqueous Ratio}

Much like the 1:1 O/A ratio, the break-time tests indicated a system suitable for introduction into mixer settlers and an organic continuous phase. Experiments indicated again that the organic phase presented with cloudiness, bubbles, and entrainment while the aqueous phase remained clear. Again the dispersion numbers of $1.50 * 10^{-3}$ and $1.42 * 10^{-3}$ indicated that the system would mix and separate adequately in industrial mixer settlers. ${ }^{(2)}$

\section{1:2 Organic/Aqueous Ratio}

The 1:2 O/A presented differently than the 1:1 and 2:1 O/A ratios. In the 1:2 tests, large bubbles were observed falling down through the dispersion band into the aqueous phase. Also, unlike the other tests, the organic phase was not cloudy. No entrainment was seen in the organic phase, and bubbles did not form in the organic phase. These observations led to the conclusion that the 1:2 O/A system was aqueous continuous instead of organic continuous. Again, the dispersion numbers of $1.64 * 10-3$ and $1.47 * 10-3$ indicate a system that can be used in an industrial mixer settler.

\subsection{Hydraulic Test}

\section{1:1 Organic/Aqueous Ratio}

For a 1:1 O/A ratio at RPMs in the range of 2000 to 3000 , no mixing was thought to occur as the TBP/Dodecane outlet stream did not cloud as observed when known mixing was 
occurring. Also, no aqueous phase was observed in any of the light phase outlet samples taken for that range of rotor speeds, regardless of throughput. Mixing and two distinct phases were observed in samples taken from the organic outlet for a $700 \mathrm{ml} / \mathrm{min}$ throughput. For RPMs from 5600 to 4800 the aqueous phase in the organic sample was determined to be less than $1.0 \%$ of the total volume of the sample. At 4700 RPMs samples from the light phase outlet contained a heavy phase or aqueous bubble determined to be $0.92 \%$ of the total volume of the sample. When the RPM was lowered to 4600 RPMs the aqueous phase in the light phase outlet sample jumped to $2.91 \%$ of the total volume, which was considered failure. $4700 \mathrm{RPMs}$ and $700 \mathrm{ml} / \mathrm{min}$ throughput was subsequently marked as the first point, being that it was the lowest RPM at this total throughput where separation failure was still below one percent. The contactor pumps were then set to deliver $800 \mathrm{ml} / \mathrm{min}$ of total throughput at an O/A ratio of 1:1. For RPMs at and above 4900 the light phase samples contained less than one percent contamination of the heavy phase.

At 4800 RPMs the contamination of the heavy phase in the light phase samples was measured as $3.57 \%$ of the total sample volume. The total throughput was then increased to $900 \mathrm{ml} / \mathrm{min}$. For RPMs ranging from 5900 to 5100 the cross contamination of the heavy phase into the light phase was determined to be less than one percent according to samples taken from the light phase outlet at each RPM setting. At an RPM setting of 5000 the aqueous phase contamination into the light phase samples became $1.0 \%$ which was considered the fail point. At $1000 \mathrm{ml} / \mathrm{min}$ total throughput the failure point was reached at 5400 RPMs where the contamination of the heavy phase into the light phase samples became $1.17 \%$. Any throughput larger that $1000 \mathrm{ml} / \mathrm{min}$ could not be separated effectively as the highest RPM available for the contactor (5900 RPM) still had a heavy phase of $9.3 \%$ of the total light phase sample volume at a throughput of $1100 \mathrm{ml} / \mathrm{min}$. 
Samples from the heavy phase outlet where observed to have no significant amount of organic light phase contamination by the absence of any considerable amount of organic film formed on top of the samples.

\section{2:1 Organic/Aqueous Ratio}

As described the same procedures were followed with the difference that the organic to aqueous ratio was set to $2: 1$. With a total throughput of $750 \mathrm{ml} / \mathrm{min}$, RPMs were decreased in increments of 100 as before. The first fail point was at 4700 RPMs where the cross contamination of heavy phase into the light phase was determined $1.82 \%$ based on samples of the light phase outlet. At $810 \mathrm{ml} / \mathrm{min}$ total throughput the fail point was determined to be 4900 RPMs where the heavy phase was $1.82 \%$ of the total volume of the light phase outlet sample. For a total throughput of $930 \mathrm{ml} / \mathrm{min}$ the fail point was determined to be at $5200 \mathrm{RPMs}$ where the percentage of heavy phase in the light phase sample was $1.00 \%$. At $990 \mathrm{ml} / \mathrm{min}$ the fail point was determined to be at 5300 RPMs where the percentage of heavy phase in the light phase sample was $1.41 \%$. Again, all heavy phase samples showed no significant amount of light phase contamination. A no mixing effect described previously was observed for low total throughputs and low RPM's in the ranges not described in the hydraulic curve in Figure A. Again, no separation was possible for total throughputs greater than $1000 \mathrm{ml} / \mathrm{min}$.

\section{1:2 Organic/Aqueous Ratio}

With the 0.95 inch weir used in the previous two hydraulic tests separation failed at all throughputs and RPMs with the organic to aqueous ratio set to 1:2 The weir was removed and 
replaced with a 1.00 inch weir. With the new weir in place, very little heavy phase contamination was observed, $<0.06 \%$, with a total throughput of $750 \mathrm{ml} / \mathrm{min}$ in all RPM ranges. At $810 \mathrm{ml} / \mathrm{min}$ total throughput, a heavy phase contamination of the light phase was observed at 4700 RPMs, but only at $0.28 \%$. All other RPMs either showed no mixing for the RPMs below 3500 or very little heavy phase contamination, $<0.01 \%$, of the light phase samples. Since no phase contamination occurred at or above 4700 RPMs, this point was taken as the first at which separation failure was less than $1.0 \%$. At a total throughput of $900 \mathrm{ml} / \mathrm{min}$, an RPM setting of 5300 produced a light phase sample with a heavy phase contamination of $0.56 \%$ of the total sample volume. At 5200 RPMs the heavy phase contamination in the light phase sample was determined to be $5.22 \%$ of the total sample volume. No significant amount of light phase was observed in the heavy phase outlet collection for the 1:2 O/A ratio. A throughput of $990 \mathrm{ml} / \mathrm{min}$ resulted in complete failure at the highest RPM setting.

Figure 6-1 below shows the results of the hydraulic curve for each of the O/A ratios. The points plotted are those where separation failure was still below 1.0\%, but for any RPM below the curve separation failed to be less than $1.0 \%$. The curve was used in determining the operation parameters of the following extraction tests. Each extraction test was performed at a particular throughput with an RPM setting of 100 RPMs above the curve. 


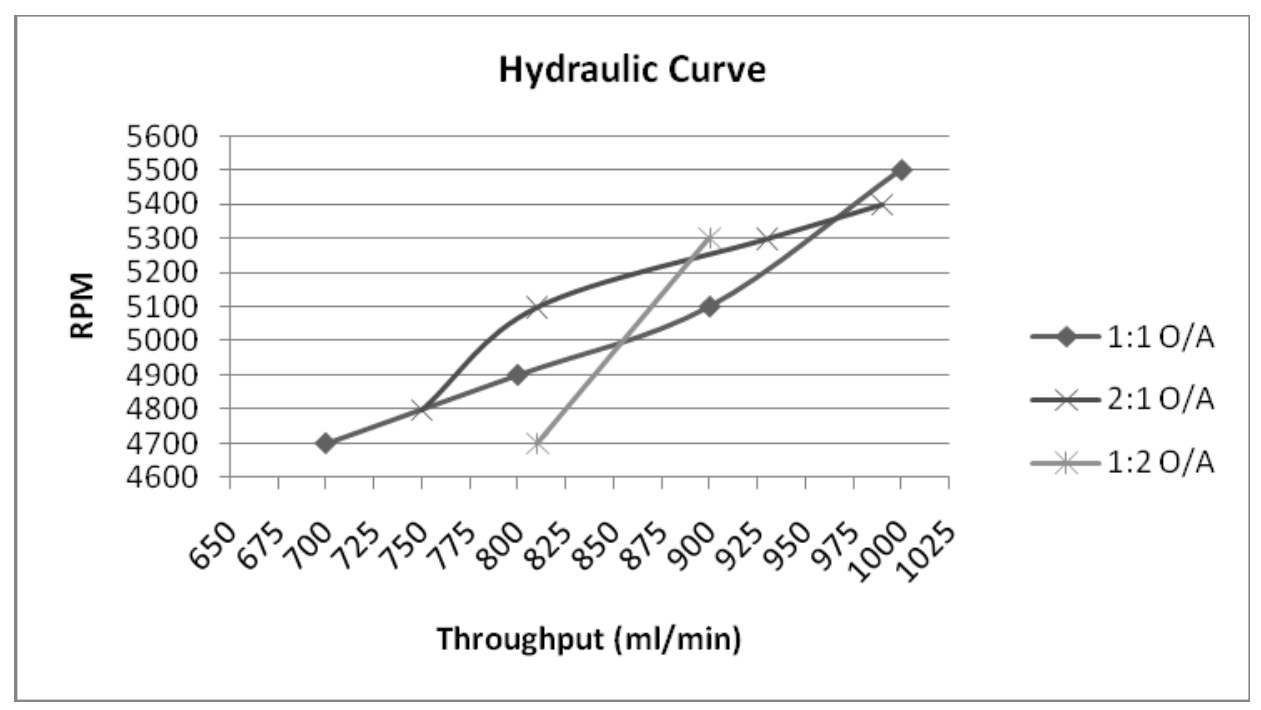

Figure 6-1. Operational Hydraulic Curve for determining points where the contactor fails to separate effectively

\subsection{Extraction Test}

(Tables given in Appendix 2)

Extraction tests were performed at both a "low" and "high" throughput for each O/A ratio. Determination of the exact total throughput values was based on the hydraulic curve.

\section{1:1 O/A (800 ml/min $5000 \mathrm{RPM})$}

The results from the equilibrium tests results used in efficiency calculations for the 800 $\mathrm{ml} / \mathrm{min}$ throughput for an organic ratio of one are shown in Table 6-2. The molarity of acetic acid and nitric acid left in the aqueous phase were determined to be 0.029 and 0.426 moles/liter respectively through the pre-described titration method. Subtraction of the number of moles from the initial acid molarity gave the number of moles extracted by the TBP/Dodecane phase. This allowed for determination of the distribution coefficients for the acids to by dividing the number of moles of acid in the organic TBP/Dodecane phase by the number of moles of acid in the 
aqueous phase at equilibrium. A distribution of 0.703 was determined for acetic acid and 0.223 for nitric acid.

The contactor study results for extraction based on an organic ratio of one at $800 \mathrm{ml} / \mathrm{min}$ with a rotor speed of 5000 rotations per minute are found in Table 6-3. Samples from the aqueous raffinate were taken and put through the same calculations as the equilibrium aqueous phase. The contactor samples produced an acetic acid distribution of 0.743 and nitric acid distribution of 0.223 . When the contactor results were compared to the equilibrium conditions, contactor efficiency for acetic acid removal was determined to be $105.7 \pm 1.9 \%$. The contactor's efficiency for nitric acid removal was determined to be $99.6 \pm .85 \%$. Efficiency results are shown in Table 6-4.

\section{1:1 O/A (1000ml/min $5600 \mathrm{RPM})$}

Upon completion of the lower total throughput for an O/A of 1:1, the same TBP/Dodecane used in the test was stripped and cleaned with $\mathrm{NaOH}$ and deionized water to remove the acetic and nitric acid extracted during the previous tests. Once stripped of acid, the organic solvent was used in another equilibrium test in order to observe if the stripping was effective and in order to use the new distribution as a basis for an extraction test at a higher total throughput with a 1:1 O/A ratio. The observed distribution of acetic acid in the second equilibrium test was 0.716 compared to 0.703 for the first. This determined that the stripping was effective and a distribution of 0.716 would be used to calculate efficiency in the following contactor trial. The nitric acid distribution observed in the second equilibrium test was 0.214 compared to 0.223 for the first. Again, the stripping was determined effective and a distribution 
of 0.214 was used in a contactor trial with a higher total throughput than the previous test. Equilibrium results for this test can be found in Table 6-5.

A total throughput of $1000 \mathrm{ml} / \mathrm{min}$ at a rotor speed of 5600 was used to determine the extent acetic and nitric acid was extracted at said throughput and an $\mathrm{O} / \mathrm{A}$ ratio of 1:1. The distribution of acetic acid was 0.678 . Comparisons to the distribution of 0.716 found in the equilibrium test determined the efficiency of the contactor for removing acetic acid at the given conditions to be $94.7 \pm 1.48 \%$. The nitric acid distribution observed with this flow scheme was 0.221. Again, compared to the equilibrium results of 0.214 , contactor efficiency for removing nitric acid at these conditions was determined to be $103.2 \pm 1.32 \%$. Results for this extraction test can be found in Table 6-6 and Table 6-7.

\section{$2: 1 \mathrm{O} / \mathrm{A}(810 \mathrm{ml} / \mathrm{min} 5000 \mathrm{RPM})$}

The same procedures used in the previous tests were again followed for an organic ratio of two to one. The new equilibrium results were expected to be much different since twice the volume of organic to aqueous was used. Having more tributylphosphate complexes, and therefore more bonding sites, in the system would allow for an increase in the extraction of acid molecules from the aqueous system. This prediction was observed in the equilibrium test as the distribution of acetic was determined to be 1.235 and the distribution of nitric was determined to be 0.352 , almost twice more than what was observed with the equal volume tests. The results for this equilibrium test can be found in Table 6-8. 
Contactor results with a flow rate of organic doubling the flow rate of aqueous produced a distribution of acetic acid at 1.218 and a distribution of nitric acid at 0.357 . Comparing these results to the equilibrium results produced contactor efficiencies at $98.6 \pm 2.35 \%$ and $101.4 \pm 0.41 \%$ for the acetic and nitric respectively at the lower total throughput. Table $6-9$ and Table 6-10 shows the results of the contactor test with an $\mathrm{O} / \mathrm{A}$ of $2: 1$, a throughput of $810 \mathrm{ml} / \mathrm{min}$ and a rotor speed of $5000 \mathrm{RPM}$.

\section{2:1 O/A (990ml/min 5500 RPM)}

Another equilibrium test with twice the organic to aqueous volume produced an acetic distribution of 1.452 and a nitric distribution of 0.385 . These results can be found in Table 6-11.

The contactor trial for the higher total throughput with an $\mathrm{O} / \mathrm{A}$ ratio of 2:1 produced an acetic distribution of 1.489 and a nitric distribution of 0.419 . Contactor efficiency based on the distribution of the equilibrium tests were determined to be $102.6 \pm 2.05 \%$ for acetic acid and 109.0 $0.48 \%$ for nitric acid. Results for this test can be found in Table 6-12 and Table 6-13.

\section{$1: 2 \mathrm{O} / \mathrm{A}(810 \mathrm{ml} / \mathrm{min} 4800 \mathrm{RPM})$}

Table 6-14 shows the results for the equilibrium test performed with an organic volume half of the aqueous volume. An expected lower distribution was observed for each of the acids. The acetic acid distribution was found to be 0.429 and the nitric acid distribution determined to be 0.108 . 
Because the lowered flow rate of the organic solvent eliminated the need for scrubbing the organic between tests, both contactor studies for the lower total throughput and the higher total throughput with a 1:2 O/A ratio were able to be performed on the same day with the higher total throughput test immediately following the lower total throughput test. This allowed for the previous equilibrium test to be used as a basis for both 1:2 O/A contactor efficiency tests. For the lower total throughput an acetic distribution of 0.372 and a nitric distribution of 0.118 were determined. Contactor efficiency based on the equilibrium test was found to $86.6 \pm 1.76 \%$ for acetic acid and $109.2 \pm 0.83 \%$ for nitric acid. The results for the extraction tests performed at an $\mathrm{O} / \mathrm{A}$ ratio of 1:2, a total throughput of $810 \mathrm{ml} / \mathrm{min}$ and a rotor speed of $4800 \mathrm{RPM}$ are found in Table 6-15 and Table 6-16.

\section{1:2 O/A (900ml/min $5400 \mathrm{RPM})$}

The higher total throughput test for an $\mathrm{O} / \mathrm{A}$ of 1:2 produced an acetic distribution of 0.306 and a nitric distribution of 0.118 . Contactor efficiency based on the equilibrium test was found to $71.3 \pm 0.0 \%$ for acetic acid and $109.7 \pm 0.24 \%$ for nitric acid. The results for the extraction tests performed at an $\mathrm{O} / \mathrm{A}$ ratio of $1: 2$, a total throughput of $900 \mathrm{ml} / \mathrm{min}$ and a rotor speed of 5400 RPM are found in Table 6-18 and Table 6-19. 


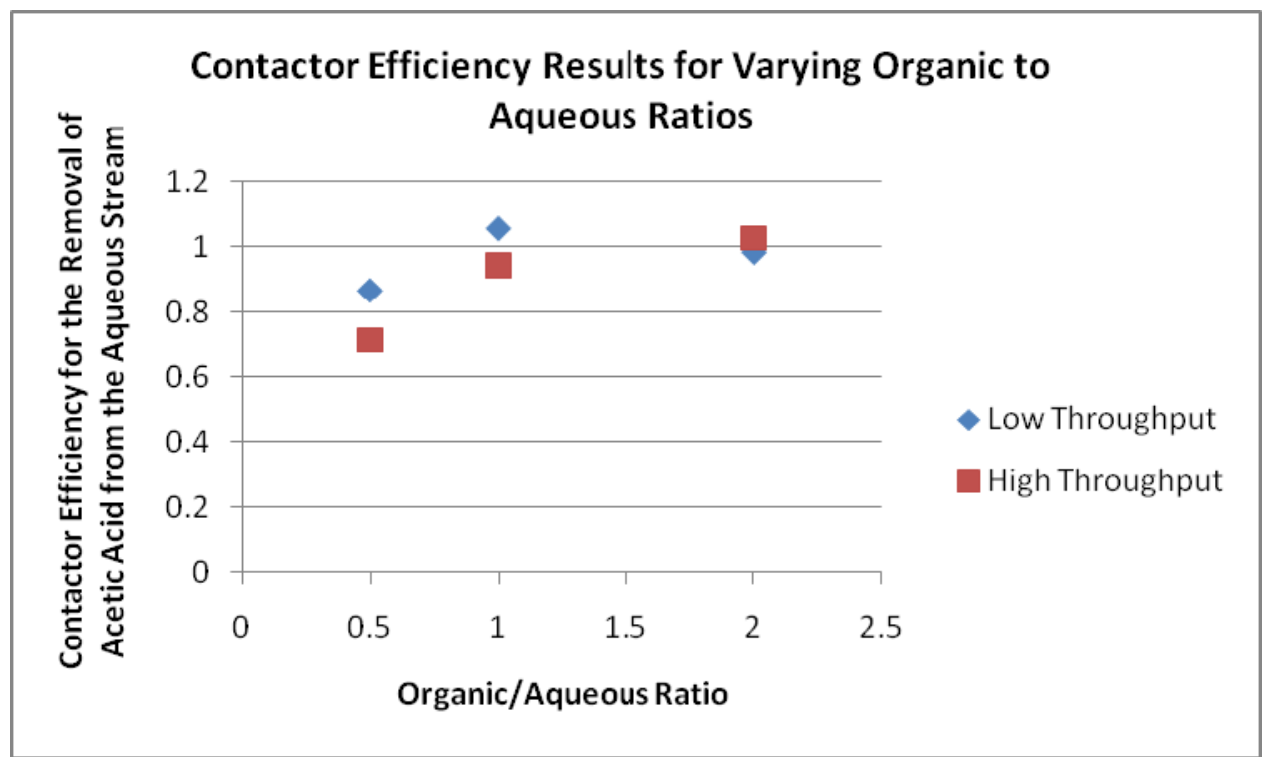

Figure 6-2. Graph showing the results for extraction efficiency tests based on equilibrium conditions

Figure 6-2 depicts the results of each of the efficiency tests as a result of the total throughput. Efficiencies were somewhat similar for organic ratios of 1 and 2 however the distributions were much higher for the 2:1 O/A ratio as can be seen in Figure 6-3. Standard deviations for the samples taken from the contactor raffinate ranged on the order of magnitude from $10^{-2}$ to $10^{-4}$ even though samples were taken at different time intervals and showed no clear distinct trend as a function of time. 


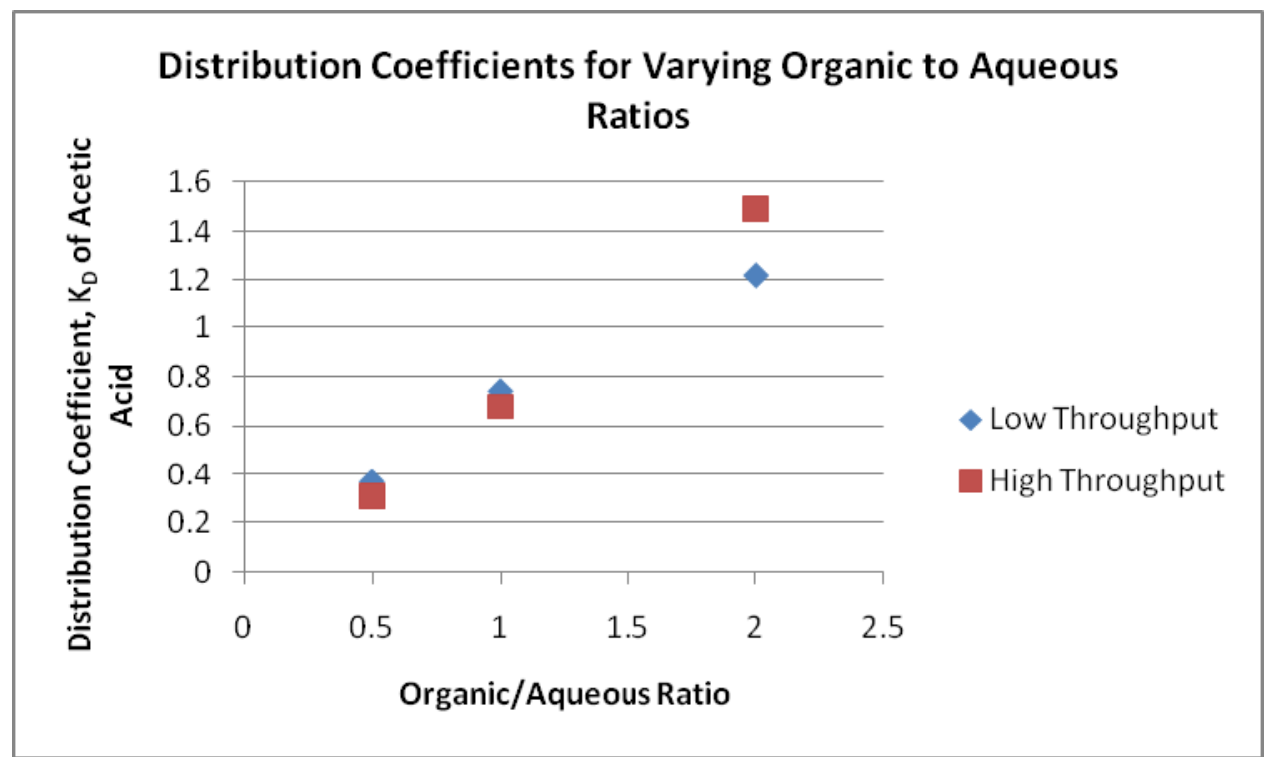

Figure 6-3. Distribution Coefficients taken from contactor extraction test results

\section{Conclusions}

After using the unitless dispersion number to determine that the $1: 1,2: 1$, and $1: 2 \mathrm{O} / \mathrm{A}$ ratios were suitable for use in an industrial mixer settler, the hydraulic tests showed some interesting results. The hydraulic tests indicated that the contactor motor in the system needed to be run at RPMs greater than those normally seen with other annular centrifugal contactors. One possible explanation for the increased RPMs required for this system could be the increased molarity of the TBP/n-dodecane organic phase. In these tests, the molarity was $1.5 \mathrm{M}$ TBP whereas other previous tests have been run at lower molarities near $1.1 \mathrm{M} \mathrm{TBP}{ }^{(3)}$ This TBP concentration increase could lead to an increase in the required RPMs for mixing and separation.

Once the hydraulic tests were concluded, the extraction testing was performed. It appears that in the organic continuous systems, the extraction efficiency is near $100 \%$ or greater for both 
acetic and nitric acid. However, in the aqueous continuous system, 1:2 O/A ratio, the acetic acid efficiency dropped off significantly while the nitric efficiency remained high. This difference in acetic acid extraction efficiency could be a result of the phase continuity shift.

To this point, complete explanation of the greater than $100 \%$ efficiencies has been difficult. There are many possible explanations ranging from plastics in the tubing leaching into the streams to increased temperature of the dispersion inside the mixing zone or possibly some system specific kinetic effects. This problem could be researched more in the future to gain insight into the actual phenomena at work.

\section{Acknowledgement}

The authors thank Joseph Birdwell, Jessica Mitchell, Christina Karni, and Jon Garrison for their support, advice and hard work during the course of this study. This research was supported by the U.S. Department of Energy, NERI program, under DOE Contract No. DEPS07-05ID14713.

\section{References}

(1) Mitchell, J.; Watson, J.S.; Johnson, J; Spencer, B.B.; Counce, R.M.; Del Cul, G.D. Extracting Acetic Acid from Acidic Solutions. Separation Science and Technology. 2008. 43(9). 2537.

(2) Ralph Leonard (dispersion number paper)

(3) Joseph Birdwell. Private Communication. July 2008. 
(4) Terry Todd, Joe Birdwell, (other contactor hydraulic curves) 


\section{Appendix 1}

Table 6-1. Batch-test data for $1: 1,1: 2$, and $2: 1 \mathrm{O} / \mathrm{A}$ ratios

\begin{tabular}{|c|c|c|c|}
\hline \multicolumn{4}{|c|}{ Aqueous 0.05 M Acetic Acid / 0.5 M Nitric Acid } \\
\hline \multicolumn{4}{|c|}{ Organic 1.5 M TBP in Dodecane } \\
\hline \multicolumn{4}{|c|}{ Organic to Aqueous Ratio - 1:1 } \\
\hline Dispersion Band Height & $17.60 \mathrm{~cm}$ & Dispersion Band Height & $17.90 \mathrm{~cm}$ \\
\hline Interface Height & $8.50 \mathrm{~cm}$ & Interface Height & $8.90 \mathrm{~cm}$ \\
\hline Final Interface Height & 8.20 & Final Interface Height & $8.70 \mathrm{~cm}$ \\
\hline Time to Interface & 102.35 & Time to Interface & 101.87 \\
\hline Dispersion Number & $1.31 E-03$ & Dispersion Number & $1.33 \mathrm{E}-03$ \\
\hline \multicolumn{4}{|c|}{ Organic to Aqueous Ratio - 1:2 } \\
\hline Dispersion Band Height & $17.40 \mathrm{~cm}$ & Dispersion Band Height & $17.80 \mathrm{~cm}$ \\
\hline Interface Height & $11.30 \mathrm{~cm}$ & Interface Height & $11.70 \mathrm{~cm}$ \\
\hline Final Interface Height & $11.25 \mathrm{~cm}$ & Final Interface Height & $11.60 \mathrm{~cm}$ \\
\hline Time to Interface & 88.88 & Time to Interface & 94.89 \\
\hline Dispersion Number & $1.50 \mathrm{E}-03$ & Dispersion Number & $1.42 \mathrm{E}-03$ \\
\hline \multicolumn{4}{|c|}{ Organic to Aqueous Ratio - 2:1 } \\
\hline Dispersion Band Height & $17.50 \mathrm{~cm}$ & Dispersion Band Height & $17.60 \mathrm{~cm}$ \\
\hline Interface Height & $5.20 \mathrm{~cm}$ & Interface Height & $5.60 \mathrm{~cm}$ \\
\hline Final Interface Height & $5.60 \mathrm{~cm}$ & Final Interface Height & $5.30 \mathrm{~cm}$ \\
\hline Time to Interface & 81.25 & Time to Interface & 91.09 \\
\hline Dispersion Number & $1.64 \mathrm{E}-03$ & Dispersion Number & $1.47 \mathrm{E}-03$ \\
\hline
\end{tabular}




\section{Appendix 2}

\section{1:1 O/A (800 ml/min 5000 RPM)}

Table 6-2. Equilibrium test results for a 1:1 organic to aqueous ratio used as a basis for the $800 \mathrm{ml} / \mathrm{min}$ total throughput test on the contactor

\begin{tabular}{|c|c|}
\hline \multicolumn{2}{|c|}{ 1:1 O/A Equilibrium Results } \\
\hline Acetic Molarity in Aqueous & 0.029 \\
\hline Nitric Molarity in Aqueous & 0.426 \\
\hline Acetic In Organic & 0.020 \\
\hline Acetic Fraction Extracted & 0.413 \\
\hline Nitric in Organic & 0.095 \\
\hline Nitric Fraction Extracted & 0.183 \\
\hline Acetic Distribution & 0.703 \\
\hline Nitric Distribution & 0.223 \\
\hline
\end{tabular}

Table 6-3. Sample averages results with standard deviations for $800 \mathrm{ml} / \mathrm{min}$ total throughput test

\begin{tabular}{|c|c|c|c|c|}
\hline \multicolumn{2}{|c|}{ Sample Averaqes } & STDev & Lower Bound & Upper Bound \\
\hline Acetic Molarity in Aqueous & 0.028 & $5.716 \mathrm{E}-04$ & 0.028 & 0.028 \\
\hline Nitric Molarity in Aqueous & 0.426 & $1.715 \mathrm{E}-03$ & 0.426 & 0.427 \\
\hline Acetic In Organic & 0.021 & $5.716 \mathrm{E}-04$ & 0.021 & 0.021 \\
\hline Acetic Fraction Extracted & 0.426 & $1.163 \mathrm{E}-02$ & 0.422 & 0.431 \\
\hline Nitric in Organic & 0.095 & $1.715 \mathrm{E}-03$ & 0.094 & 0.096 \\
\hline Nitric Fraction Extracted & 0.182 & $3.291 \mathrm{E}-03$ & 0.181 & 0.183 \\
\hline Acetic Distribution & 0.743 & $3.531 \mathrm{E}-02$ & 0.729 & 0.756 \\
\hline Nitric Distribution & 0.223 & $4.934 \mathrm{E}-03$ & 0.221 & 0.224 \\
\hline
\end{tabular}


Table 6-4. Contactor Efficiency for the removal of acetic acid and nitric acid at $800 \mathrm{ml} / \mathrm{min}$ total throughput

Lower Bound Upper Bound

Acetic Efficiency

$103.7 \%$

$98.8 \%$

$107.5 \%$

Nitric Efficiency

$=\quad 98.8 \%$

$100.5 \%$

\section{1:1 O/A (1000ml/min 5600 RPM)}

Table 6-5. Equilibrium test results for a 1:1 organic to aqueous ratio used as a basis for the $1000 \mathrm{ml} / \mathrm{min}$ total throughput test on the contactor

\begin{tabular}{|c|c|}
\hline \multicolumn{2}{|c|}{ 1:1 O/A Equilibrium Results } \\
\hline Acetic Molarity in Aqueous & 0.028 \\
\hline Nitric Molarity in Aqueous & 0.421 \\
\hline Acetic In Organic & 0.020 \\
\hline Acetic Fraction Extracted & 0.417 \\
\hline Nitric in Organic & 0.090 \\
\hline Nitric Fraction Extracted & 0.176 \\
\hline Acetic Distribution & 0.716 \\
\hline Nitric Distribution & 0.214 \\
\hline
\end{tabular}


Table 6-6. Sample averages results with standard deviations for $1000 \mathrm{ml} / \mathrm{min}$ total throughput test

\begin{tabular}{|c|c|c|c|c|}
\hline \multicolumn{2}{|c|}{ Sample Averages } & STDev & Lower Bound & Upper Bound \\
\hline Acetic Molarity in Aqueous & 0.028 & $4.590 \mathrm{E}-04$ & 0.028 & 0.029 \\
\hline Nitric Molarity in Aqueous & 0.419 & $2.517 \mathrm{E}-03$ & 0.418 & 0.420 \\
\hline Acetic In Organic & 0.019 & $4.590 \mathrm{E}-04$ & 0.019 & 0.019 \\
\hline Acetic Fraction Extracted & 0.404 & $9.649 \mathrm{E}-03$ & 0.400 & 0.408 \\
\hline Nitric in Organic & 0.092 & $2.517 \mathrm{E}-03$ & 0.091 & 0.093 \\
\hline Nitric Fraction Extracted & 0.181 & $4.927 \mathrm{E}-03$ & 0.179 & 0.183 \\
\hline Acetic Distribution & 0.678 & $2.742 \mathrm{E}-02$ & 0.667 & 0.688 \\
\hline Nitric Distribution & 0.221 & $7.319 \mathrm{E}-03$ & 0.218 & 0.223 \\
\hline
\end{tabular}

Table 6-7. Contactor Efficiency for the removal of acetic acid and nitric acid at $1000 \mathrm{ml} / \mathrm{min}$ total throughput

Lower Bound Upper Bound

Acetic Efficiency

$93.2 \%$

$101.9 \%$

$96.2 \%$

Nitric Efficiency

$=$

$104.5 \%$ 


\section{2:1 O/A (810ml/min 5000 RPM)}

Table 6-8. Equilibrium test results for a 2:1 organic to aqueous ratio used as a basis for the $810 \mathrm{ml} / \mathrm{min}$ total throughput test on the contactor

\begin{tabular}{|c|c|}
\hline \multicolumn{2}{|c|}{ 2:1 O/A Equilibrium Results } \\
\hline Acetic Molarity in Aqueous & 0.021 \\
\hline Nitric Molarity in Aqueous & 0.367 \\
\hline Acetic In Organic & 0.026 \\
\hline Acetic Fraction Extracted & 0.553 \\
\hline Nitric in Organic & 0.129 \\
\hline Nitric Fraction Extracted & 0.260 \\
\hline Acetic Distribution & 1.235 \\
\hline Nitric Distribution & 0.352 \\
\hline
\end{tabular}

Table 6-9. Sample averages results with standard deviations for $810 \mathrm{ml} / \mathrm{min}$ total throughput test

\begin{tabular}{|c|c|c|c|c|}
\hline \multicolumn{2}{|c|}{ Sample Averages } & STDev & Lower Bound & Upper Bound \\
\hline Acetic Molarity in Aqueous & 0.021 & $7.249 \mathrm{E}-04$ & 0.021 & 0.022 \\
\hline Nitric Molarity in Aqueous & 0.366 & $9.998 \mathrm{E}-04$ & 0.365 & 0.366 \\
\hline Acetic In Organic & 0.026 & $7.249 \mathrm{E}-04$ & 0.026 & 0.026 \\
\hline Acetic Fraction Extracted & 0.549 & $1.532 \mathrm{E}-02$ & 0.543 & 0.555 \\
\hline Nitric in Organic & 0.130 & $9.998 \mathrm{E}-04$ & 0.130 & 0.131 \\
\hline Nitric Fraction Extracted & 0.263 & $2.014 \mathrm{E}-03$ & 0.262 & 0.264 \\
\hline Acetic Distribution & 1.218 & $7.517 \mathrm{E}-02$ & 1.189 & 1.247 \\
\hline Nitric Distribution & 0.357 & $3.712 \mathrm{E}-03$ & 0.355 & 0.358 \\
\hline
\end{tabular}


Table 6-10. Contactor Efficiency for the removal of acetic acid and nitric acid at $810 \mathrm{ml} / \mathrm{min}$ total throughput

Lower Bound Upper Bound

\begin{tabular}{|cccc|}
\hline Acetic Efficiency & $=$ & $96.3 \%$ & $101.0 \%$ \\
\hline Nitric Efficiency & $=$ & $101.0 \%$ & $101.9 \%$ \\
\hline
\end{tabular}

\section{2:1 O/A (990ml/min 5500 RPM)}

Table 6-11. Equilibrium test results for a 2:1 organic to aqueous ratio used as a basis for the $990 \mathrm{ml} / \mathrm{min}$ total throughput test on the contactor

\begin{tabular}{|c|c|}
\hline \multicolumn{2}{|c|}{ 2:1 O/A Equilibrium Results } \\
\hline Acetic Molarity in Aqueous & 0.021 \\
\hline Nitric Molarity in Aqueous & 0.367 \\
\hline Acetic In Organic & 0.031 \\
\hline Acetic Fraction Extracted & 0.592 \\
\hline Nitric in Organic & 0.141 \\
\hline Nitric Fraction Extracted & 0.278 \\
\hline Acetic Distribution & 1.452 \\
\hline Nitric Distribution & 0.385 \\
\hline
\end{tabular}


Table 6-12. Sample averages results with standard deviations for $990 \mathrm{ml} / \mathrm{min}$ total throughput test

\begin{tabular}{|c|c|c|c|c|}
\hline \multicolumn{2}{|c|}{ Sample Averages } & $\underline{\text { STDev }}$ & Lower Bound & Upper Bound \\
\hline Acetic Molarity in Aqueous & 0.021 & $6.446 \mathrm{E}-04$ & 0.021 & 0.021 \\
\hline Nitric Molarity in Aqueous & 0.358 & $1.213 \mathrm{E}-03$ & 0.358 & 0.358 \\
\hline Acetic In Organic & 0.031 & $6.446 \mathrm{E}-04$ & 0.031 & 0.031 \\
\hline Acetic Fraction Extracted & 0.598 & $1.244 \mathrm{E}-02$ & 0.593 & 0.603 \\
\hline Nitric in Organic & 0.150 & $1.213 \mathrm{E}-03$ & 0.150 & 0.151 \\
\hline Nitric Fraction Extracted & 0.295 & $2.388 \mathrm{E}-03$ & 0.295 & 0.296 \\
\hline Acetic Distribution & 1.489 & $7.705 \mathrm{E}-02$ & 1.460 & 1.519 \\
\hline Nitric Distribution & 0.419 & $4.806 \mathrm{E}-03$ & 0.418 & 0.421 \\
\hline
\end{tabular}

Table 6-13. Contactor Efficiency for the removal of acetic acid and nitric acid at $990 \mathrm{ml} / \mathrm{min}$ total throughput

Lower Bound Upper Bound

\begin{tabular}{|cccc|}
\hline Acetic Efficiency & $=$ & $100.5 \%$ & $104.6 \%$ \\
\hline Nitric Efficiency & $=$ & $108.5 \%$ & $109.5 \%$ \\
\hline
\end{tabular}




\section{1:2 O/A (810ml/min 4800 RPM)}

Table 6-14. Equilibrium test results for a 1:2 organic to aqueous ratio used as a basis for the $810 \mathrm{ml} / \mathrm{min}$ total throughput test on the contactor

\begin{tabular}{|c|c|}
\hline \multicolumn{2}{|c|}{ 1:2 O/A Equilibrium Results } \\
\hline Acetic Molarity in Aqueous & 0.035 \\
\hline Nitric Molarity in Aqueous & 0.446 \\
\hline Acetic In Organic & 0.015 \\
\hline Acetic Fraction Extracted & 0.300 \\
\hline Nitric in Organic & 0.048 \\
\hline Nitric Fraction Extracted & 0.097 \\
\hline Acetic Distribution & 0.429 \\
\hline Nitric Distribution & 0.108 \\
\hline
\end{tabular}

Table 6-15. Sample averages results with standard deviations for $810 \mathrm{ml} / \mathrm{min}$ total throughput, 1:2 O/A test

\begin{tabular}{|c|c|c|c|c|}
\hline \multicolumn{2}{|c|}{ Sample Averages } & STDev & Lower Bound & Upper Bound \\
\hline Acetic Molarity in Aqueous & 0.037 & $5.227 \mathrm{E}-04$ & 0.037 & 0.037 \\
\hline Nitric Molarity in Aqueous & 0.443 & $9.147 \mathrm{E}-04$ & 0.442 & 0.443 \\
\hline Acetic In Organic & 0.014 & $5.227 \mathrm{E}-04$ & 0.013 & 0.014 \\
\hline Acetic Fraction Extracted & 0.271 & $1.036 \mathrm{E}-02$ & 0.267 & 0.275 \\
\hline Nitric in Organic & 0.052 & $9.147 \mathrm{E}-04$ & 0.052 & 0.052 \\
\hline Nitric Fraction Extracted & 0.105 & $1.850 \mathrm{E}-03$ & 0.104 & 0.106 \\
\hline Acetic Distribution & 0.372 & $1.950 \mathrm{E}-02$ & 0.364 & 0.380 \\
\hline Nitric Distribution & 0.118 & $2.310 \mathrm{E}-03$ & 0.117 & 0.118 \\
\hline
\end{tabular}


Table 6-16. Contactor Efficiency for the removal of acetic acid and nitric acid at $810 \mathrm{ml} / \mathrm{min}$ total throughput, 1:2 O/A

Lower Bound Upper Bound

Acetic Efficiency $84.9 \%$ $88.4 \%$ Nitric Efficiency $108.4 \%$

$110.1 \%$

1:2 O/A (900ml/min $5400 \mathrm{RPM})$

Table 6-17. Equilibrium test results for a 1:2 organic to aqueous ratio used as a basis for the $900 \mathrm{ml} / \mathrm{min}$ total throughput test on the contactor

\begin{tabular}{|c|c|}
\hline \multicolumn{2}{|c|}{ 1:2 O/A Equilibrium Results } \\
\hline Acetic Molarity in Aqueous & 0.035 \\
\hline Nitric Molarity in Aqueous & 0.446 \\
\hline Acetic In Organic & 0.015 \\
\hline Acetic Fraction Extracted & 0.300 \\
\hline Nitric in Organic & 0.048 \\
\hline Nitric Fraction Extracted & 0.097 \\
\hline Acetic Distribution & 0.429 \\
\hline Nitric Distribution & 0.108 \\
\hline
\end{tabular}


Table 6-18. Sample averages results with standard deviations for $900 \mathrm{ml} / \mathrm{min}$ total throughput, 1:2 O/A test

\begin{tabular}{|c|c|c|c|c|}
\hline \multicolumn{2}{|c|}{ Sample Averages } & STDev & Lower Bound & Upper Bound \\
\hline Acetic Molarity in Aqueous & 0.039 & $0.000 \mathrm{E}+00$ & 0.039 & 0.039 \\
\hline Nitric Molarity in Aqueous & 0.442 & $2.613 \mathrm{E}-04$ & 0.442 & 0.442 \\
\hline Acetic In Organic & 0.012 & $0.000 \mathrm{E}+00$ & 0.012 & 0.012 \\
\hline Acetic Fraction Extracted & 0.234 & $0.000 \mathrm{E}+00$ & 0.234 & 0.234 \\
\hline Nitric in Organic & 0.052 & $2.613 \mathrm{E}-04$ & 0.052 & 0.052 \\
\hline Nitric Fraction Extracted & 0.106 & $5.285 \mathrm{E}-04$ & 0.106 & 0.106 \\
\hline Acetic Distribution & 0.306 & $0.000 \mathrm{E}+00$ & 0.306 & 0.306 \\
\hline Nitric Distribution & 0.118 & $6.609 \mathrm{E}-04$ & 0.118 & 0.119 \\
\hline
\end{tabular}

Table 6-19. Contactor Efficiency for the removal of acetic acid and nitric acid at $900 \mathrm{ml} / \mathrm{min}$ total throughput, 1:2 O/A

Lower Bound Upper Bound

\begin{tabular}{|cccc|}
\hline Acetic Efficiency & $=$ & $71.3 \%$ & $71.3 \%$ \\
\hline Nitric Efficiency & $=$ & $109.7 \%$ & $110.1 \%$ \\
\hline
\end{tabular}




\section{CHAPTER VII: CONCLUSIONS}




\section{FUTURE WORK}

Savannah River National Laboratory is examining new ways to suppress plutonium extraction in the UREX step of the UREX+ process. This is a preventative approach to the removal of acetic acid. Instead of using acetohydroxamic acid which breaks down into acetic acid and hydroxyl amine nitrate, this study plans to find a new complexant/extractant that will accomplish the same goal, but break down into gaseous compounds eliminating the need for a removal step of the hydrolysis products. [2]

\section{CONCLUSIONS}

Out of the technologies discussed, the optimal method for the UREX+ process appears to be solvent extraction. Utilizing solvent extraction, acetic acid may be favorably extracted from the aqueous phase with thorough examination and selection of a solvent. From the many diluents examined, it was experimentally determined that the most capable solvents for the extraction of acetic acid are TBP in FS-13 and TBP in dodecane. The data comparing acid extraction to water content in the TBP/n-Dodecane system seems to suggest that there is a similar mechanism for the equilibrium acid extraction and water content, even though they are not extracted in the same amount. The data advocates that the water content of the organic mixture after contact is greatly independent of the diluent used, and also shows that the addition of equal volumes of nitric and acetic acid have little effect on the amount of water absorbed at equilibrium. This uniformity supports an independence from any acid co-extraction. 
With intense literature review of each step of the UREX+ process and analysis of acetic acid extraction compared with radioactive metal extraction, it was verified that the presence of acetic acid should not present any problem for the steps of the process. The primary obstruction that acetic acid poses is in the recycle of the nitric acid. It is recommended by the authors that the acetic acid solvent extraction removal step should be placed after TRUEX to relieve concerns that metals will extract with this step if placed before these metals are removed and to possibly allocate distillation as a feasible option in combination of re-concentrating nitric acid for recycle.

After utilizing break tests to determine what organic to aqueous ratios were appropriate for use in an industrial mixer settler, hydraulic tests were performed on an annular centrifugal contactor to determine what rotational speeds were appropriate for the contactor in the system. The hydraulic tests indicated that the contactor needed to be run at a greater rotational speed than normally seen with other contactors. It is believed that the increased TBP concentrations used in these tests lead to an increased need for faster mixing in order to separate. The extraction testing then demonstrated that the contactor achieves nearly $100 \%$ efficiencies when provided with equilibrium conditions.

In conclusion, the acetic acid solvent extraction removal step should be placed at the end of the UREX+ process after TRUEX. Use of a TBP-dodecane solvent will lead to the best distribution coefficients and separation factors for acetic acid and should be further explored. 


\section{REFERENCES}

1. Travis Russell, University of Tennessee, Personal Correspondence. 2008

2. Tracy Rudisill, Savannah River National Lab, Personal Correspondence, 2008. 